\title{
Positionspapier der DGHNO und der DGPP - Stand der klinischen und endoskopischen Diagnostik, Evaluation und Therapie von Schluckstörungen bei Kindern und Erwachsenen
}

\author{
Position paper of the German Society of Oto-Rhino-Laryngology, Head and Neck Surgery \\ and the German Society of Phoniatrics and Pediatric Audiology - Current State of Clinical \\ and Endoscopic Diagnostics, Evaluation, and Therapy of Swallowing Disorders in Children \\ and Adults
}

Autoren

Institute

\section{Arens ${ }^{1}$, I. F. Herrmann ${ }^{2}$, S. Rohrbach ${ }^{3}$, C. Schwemmle', T. Nawka ${ }^{3}$}

${ }^{1}$ Universitätsklinik für Hals-Nasen-Ohrenheilkunde, Universitätsklinikum Magdeburg AöR, Otto-von-Guericke-Universität, Magdeburg

${ }^{2}$ Refluxcenter, Düsseldorf

${ }^{3}$ Klinik für Audiologie und Phoniatrie, Charité - Universitätsmedizin Berlin, Berlin

Schlüsselwörter
Schlucken
Dysphagie/Ätiologie/
Diagnostik
Aspiration
Oropharynx/Pathophysiologie
Laryngoskopie
Key words
swallowing
disorders/etiology/diagnostics
aspiration
oropharynx/pathophysiology

Bibliografie

DOI http://dx.doi.org/

$10.1055 / \mathrm{s}-0035-1545298$

Laryngo-Rhino-Otol 2015; 94 :

S306-S354 @ Georg Thieme

Verlag KG Stuttgart · New York

ISSN 0935-8943

\section{Korrespondenzadressen \\ Für die DGHNO}

Prof. Dr. med. Christoph Arens

Universitätsklinik für Hals-

Nasen-Ohrenheilkunde

Universitätsklinikum

Magdeburg AöR

Leipziger Straße 44

39120 Magdeburg

christoph.arens@med.ovgu.de

\section{Für die DGPP}

Prof. Dr. med. Tadeus Nawka

Klinik für Audiologie und

Phoniatrie

Charité - Universitätsmedizin

Berlin,

Charitéplatz 1

10117 Berlin

tadeus.nawka@charite.de

\section{Inhaltsverzeichnis}

Zusammenfassung

1. Einleitung

2. Anatomische Grundlagen und Physiologie des Schluckens

2.1 Prä- und postnatales Schlucken

2.2 Phasen des Schluckens

2.2.1 Präorale oder antizipatorische Phase

2.2.2 Orale Vorbereitungsphase

2.2.3 Orale Phase

2.2.4 Pharyngeale Phase

2.2.5 Ösophageale Phase

2.3 Zentrale Steuerung des Schluckens

3. Klinik der Dysphagien

3.1 Pathophysiologie des Schluckens

3.1.1 Dysphagieeinteilung nach Lokalisation

3.1.2 Strukturveränderungen

3.1.3 Paresen

3.1.4 Hyperkinesen

3.1.5 Sensibilitätsstörungen

3.1.6 Krikopharyngeale Funktionsstörungen

3.1.7 Presbydysphagie

3.2 Erkrankungen des Kopf-Hals-Gebietes mit Dysphagie

3.2.1 Orale und pharyngeale Erkrankungen

3.2.2 Störungen der pharyngealen Phase

3.2.3 Störungen der ösophagealen Phase

3.3 Zervikogene Dysphagie

3.3.1 Funktionelle Störungen der Halswirbelsäule mit Dysphagie

3.3.2 Morphologische Ursachen der Halswirbelsäule mit Dysphagie

3.3.3 Postoperative Dysphagie bei HWS-Operationen 318

3.4

3.5

3.6

3.6.1

3.6 .2 Dysphagie durch Hypersalivation und Xerostomie 318 Neurologische Krankheitsbilder 318 Medikamentenbedingte Dysphagien 318 Lokale Wirkung auf die Schluckfunktion (oral medication-induced esophageal injury) Systemische Medikamentenwirkung auf die Schluckfunktion (Klassifikation)

3.7 Dysphagie im Kindesalter 320

3.7.1 Ursachen von Dysphagien bei Kindern 320

3.7.2 Symptomatik/Klinik von Dysphagien bei Kindern 321

3.7.3 Fütterbeobachtung, Screening und klinische Schluckuntersuchung bei Kindern

3.7.4 Standardisiertes Vorgehen bei Anamnese und Untersuchung

4. Diagnostik der Dysphagie 324

4.1 Anamnese bei Dysphagie 324

4.2 Allgemeine Beurteilung, klinische Untersuchung 324

4.2.1 Untersuchung von Lippen, Kiefer, Zunge und vorderer Mundhöhle

4.2.2 Untersuchung von hinterer Mundhöhle, Velum und Pharynx

4.3 Fragebogen-Assessments, Screeningverfahren und klinische Dysphagiediagnostik 325

4.3.1 Fragebogen-Assessments 325

4.3.2 Screeningverfahren 325

4.3.3 Untersuchung von Patienten mit Trachealkanülen 326

$4.4 \quad$ Klinische Schluckuntersuchungsverfahren 327

4.4.1 Stufenkonzepte 327

4.4.2 Endoskopische Untersuchungstechniken 327

4.5 Untersuchungsmodalitäten und Beurteilungskriterien der videoendoskopischen Untersuchung 329

4.6 Funktionsprüfungen 329

4.6.1 Prüfung des Verschlusses von Glottis und Supraglottis

4.6.2 Sensibilitätsprüfung 330

4.6.3 Überprüfung des Schluckvorgangs mit Nahrung 330

4.6.4 Überprüfung der Effektivität therapeutischer Manöver

4.6.5 Schweregrad der Aspiration und/oder der Transporteffektivität von Substanzen

4.6.6 Penetration-Aspiration Scale (PAS)

4.6.7 Flexible endoskopische Evaluation der Dysphagie (FEED)

4.6.8 FEES

4.6.9 Bogenhausener Dysphagiescore (BODS) 332

4.6.10 Einteilung der Aspirationsschweregrade 333

4.7 Die transnasale Videopanendoskopie (ViP) 333

4.8 Weitere diagnostische Verfahren 337 
5.1 Konservative Dysphagietherapie

5.1.1 Funktionelle Dysphagietherapie

5.1.2 Fazio-orale Trakt-Therapie (F.O.T.T. ${ }^{\circledR}$ )

5.1.3 Propriozeptive neuromuskuläre Faszilitation (PNF)

5.2 Therapie bei medikamentenbedingter Dysphagie

5.3 Therapie bei Hypersalivation und Xerostomie

5.3.1 Hypersalivation

5.3.2 Xerostomie

5.4 Operative Therapie der Dysphagie

5.4.1 Chirurgische Therapie spezifischer Organstrukturen

5.4.2 Spezielle Chirurgie bei besonders schwerer Aspiration

5.4.3 Chirurgische Therapie von Schluckstörungen nach Radiochemotherapie

5.4.4 Tracheotomie

5.4.5 Perkutane endoskopische Gastrostomie (PEG)

Abstract

Literatur

\section{Zusammenfassung \\ $\nabla$}

Schluckstörungen sind häufig. Sie können zu erhöhter Mortalität durch aspirationsbedingte Lungenkomplikationen und Mangelernährung einerseits sowie einer erheblichen Beeinträchtigung der Lebensqualität andererseits führen. Die demografische Entwicklung in Deutschland lässt einen kontinuierlichen Anstieg der Dysphagie in den kommenden Jahren erwarten. Schwerpunkte sind dabei neurodegenerative Erkrankungen, die Tumoren im Verlauf der Schluckstraße und die Folgen der Tumorbehandlung im oberen Aerodigestivtrakt.

Vorrangig sind die Ärzte aus dem HNO-Bereich und der Phoniatrie als kompetente Fachgruppen zentrale Ansprechpartner für die Diagnostik und Therapieplanung der Dysphagie.

Die meist komplexen Störungen verlangen eine differenzierte Anamnese, Diagnostik und eine symptomorientierte Behandlung.

Das Einbeziehen auch von nicht-ärztlichen Fachgruppen wie Logopäden, Physiotherapeuten und Ergotherapeuten in die Planung einer effizienten Therapie erweitert und komplettiert die kompetente patientenzentrierte Betreuung. Die konservative Dysphagietherapie durch Logopäden und ähnliche Fachgruppen ist, auch längerfristig, ein wichtiges Standbein der Behandlung. Zudem können Anteile der spezifischen Diagnostik in enger Kooperation übernommen werden.

Eine interdisziplinäre Zusammenarbeit insbesondere mit den intensivmedizinischen neurologischen und internistischen Fachgruppen ist erforderlich.

Die in diesem Positionspapier beschriebenen Verfahren der speziellen Endoskopie gehören zu den originären ärztlichen Leistungen, vornehmlich der Fachärzte für Hals-, Nasen-, und Ohrenheilkunde bzw. für Sprach-, Stimm- und kindliche Hörstörungen (Phoniatrie und Pädaudiologie).

Die Endoskopie ist eine ärztliche Leistung, die grundsätzlich nicht delegierbar ist „In keinem Fall delegierbar sind Leistungen der Endoskopie (außer Kapselendoskopien, die durch speziell ausgebildete nichtärztliche Mitarbeiter geführt werden können) und der Sonografie“ siehe [278]. Entsprechend ist eine Substitution des Arztes ausgeschlossen.

\section{Einleitung}

$\nabla$

Der Begriff der Dysphagie umfasst alle schmerzlosen Einschränkungen der Nahrungsaufnahme und des geregelten Transportes von Nahrung und ist eher eine Symptombeschreibung als eine Diagnose. Der Schluckvorgang als zentrale Funktion dient dem Transport von Speichel, Sekret, Flüssigkeit und Nahrung aus dem Mund über den Schlund und die Speiseröhre in den Magen. Schluckstörungen werden neben neurologischen und muskulär bedingten Erkrankungen durch tumor- oder operationsbedingte Veränderungen im Kopf-, Halsbereich, Ösophagus und Magen ausgelöst oder verstärkt [1].

Dabei spielen Lokalisation, Behandlungsart, Heilungsverlauf und Prognose der organischen Grunderkrankung eine große Rolle für das Ausmaß der Dysphagie und deren Behandelbarkeit.

In diesem Positionspapier sollen Anamnese, klinische Untersuchung, Endoskopie, diagnostische Bewertung der Befunde und Entscheidung über therapeutische Möglichkeiten und deren Konsequenzen dargestellt werden, die ärztlicherseits bei Patienten mit Dysphagie unternommen werden sollten. Die Endoskopie des oberen Aerodigestivtraktes nimmt eine Schlüsselposition in der Dysphagiediagnostik ein. Der Phoniater oder HNOArzt/Kopf- und Halschirurg beurteilt bei der Endoskopie nicht nur den funktionellen Schluckvorgang, sondern auch mögliche strukturelle Veränderungen des oberen Aerodigestivtraktes. Die flexible endoskopische Schluckuntersuchung (FESU) bildet die Grundlage des ärztlichen Handelns in diesem Positionspapier der Deutschen Gesellschaft für Hals-Nasen-Ohrenheilkunde, Kopf- und Halschirurgie (DGHNO-KHC) und der Deutschen Gesellschaft für Phoniatrie und Pädaudiologie (DGPP). Sie ist nicht gleichzusetzen mit der flexiblen endoskopischen Bewertung des Schluckens (flexible endoscopic evaluation of swallowing - FEES), ein Begriff für einen standardisierten Untersuchungsablauf mit einer systematischen Auswertung der dabei erhobenen Befunde [2].

Die flexible Endoskopie verlangt eine sichere Handhabung des Endoskops sowie profunde Kenntnisse über die Anatomie, die Physiologie und die Pathophysiologie der oberen Speise- und Luftwege. Mögliche Komplikationen bei der Vorbereitung und der Durchführung der flexiblen Endoskopie macht das Bereithalten einer entsprechenden Notfallausrüstung und den geschulten Umgang damit erforderlich. Bei der flexiblen Endoskopie sind differenzialdiagnostische Überlegungen relevant, da die Symptomatik einer Dysphagie mehrdimensional sein kann. Aus rechtlicher Sicht ergibt sich:

Die flexible Endoskopie setzt eine Aufklärung über die wesentlichen Risiken des Eingriffs und Zustimmung des Patienten voraus. Sie darf ausschließlich von Ärzten durchgeführt werden. Die flexible Endoskopie des Aerodigestivtraktes gehört zum Fachgebiet der Hals-, Nasen- und Ohrenheilkunde bzw. der Phoniatrie und Pädaudiologie [3].

\section{Anatomische Grundlagen und Physiologie des Schluckens \\ $\nabla$}

\subsection{Prä- und postnatales Schlucken}

Zwischen den ersten zu beobachtenden Schluckvorgängen in der 11. - 12. Schwangerschaftswoche (SSW) und einer weitgehenden Vervollkommnung der Schluckkompetenz mit ca. 4 Jahren liegt eine sehr komplexe strukturelle sowie funktionelle Entwicklung [4]. 
In der Embryonalperiode, 1. - 8. SSW, sind folgende Entwicklungsstufen zu beobachten: in Woche 3 bildet sich ein primitiver Mund, in Woche 4 Kiemenbögen und Schlundtaschen, aus denen sich später Gesicht, Nase, Mund, Larynx und Pharynx entwickeln.

Während der Fetalperiode, 9. SSW bis Geburt, beobachtet man folgendes: in Woche 10 ist die Glottis geöffnet, in Woche 13 - 16 beginnt das pharyngeale Schlucken mit getrennt ablaufenden Bewegungen von Lippen, Mund, Pharynx und Ösophagus. In den Wochen 17 - 20 setzen Saugbewegungen ein. In den Wochen 26 - 29 treten primitive Reflexe wie phasisches Beißen oder Würgen auf. Der Fetus stimuliert seinen Mund, reagiert mimisch auf bittere Stoffe im Fruchtwasser und schluckt bis zu $400 \mathrm{ml}$ Fruchtwasser täglich. Ab Woche 32 kann der Fetus saugen und schlucken, sodass Frühgeborene ab diesem Alter grundsätzlich gestillt werden oder Flaschennahrung zu sich nehmen können. Nach der Geburt sind frühkindliche Reflexe zu beobachten, die mit der Nahrungsaufnahme und dem Schlucken zusammenhängen. Dazu gehören Husten, Würgen, phasisches Beißen, der transversale Zungenreflex/die Protrusion, der Suchreflex (Rooting), der Greifreflex (Halten von Nahrung), der BabkinReflex (Hand-zum-Mund-Koordination) und der palmomentale Reflex (Hand-zum-Mund-Koordination). Diese primitiven Reflexe werden im Laufe des ersten Lebensjahres durch die Reifung des Gehirns sukzessive unterdrückt.

Allgemein sind am Schluckvorgang 50 Muskelpaare und 5 Hirnnerven beteiligt. Während des Schluckens setzt die Atmung aus (sogenannte „Schluckapnoe“) und der Larynx verschließt sich. Dadurch wird der Schutz der tiefen Atemwege sichergestellt.

\subsection{Phasen des Schluckens}

Die Phasen des Schluckens sind für flüssig und fest bzw. halbfest unterschiedlich. Flüssigkeiten werden von der Mundhöhle ohne Unterbrechung in die Speiseröhre geschluckt. Die Phasen des Schluckens bei festen bzw. halbfesten Speisen werden nach der jeweiligen Lokalisation des Speisebolus eingeteilt. Sie gehen ineinander über. Es werden unterschieden:

1. Präorale oder antizipatorische Phase

2. Orale Vorbereitungsphase

3. Orale Phase

4. Pharyngeale Phase

5. Ösophageale Phase

\subsubsection{Präorale oder antizipatorische Phase}

Speisen und Getränke werden vor dem Öffnen des Mundes einer Kontrolle (Geruch, Temperatur, Aussehen) unterzogen. Die Berührung der Speisen mit den Lippen rundet diese Prüfung ab.

\subsubsection{Orale Vorbereitungsphase}

In der oralen Vorbereitungsphase wird die Nahrung erneut auf Geruch, Geschmack, Temperatur und Volumen geprüft. Bei Missfallen kann der Bolus wieder ausgespuckt werden, d. h. diese Phase ist willkürlich beeinflussbar, jedoch automatisiert. Ein positives Feedback wirkt sich stimulierend auf die Schluckreflextriggerung aus. Die orale Vorbereitungsphase dient der Bolusformung und -platzierung. Zunächst erfolgt die Aufnahme, ggf. Zerkleinerung der Nahrung, und die Vermischung mit Speichel.

Die mimische Muskulatur bewirkt den suffizienten Lippenschluss u.a. durch die tonische Spannung des M. orbicularis oris; der M. buccinator reguliert den Wangentonus. Seitlich in den Mundvorhof ausgewichene Nahrungsteile werden zurück zwi- schen die Zähne geschoben. Der VII. Hirnnerv, N. facialis, innerviert die mimische Muskulatur. Das Kiefergelenk wird durch den beweglichen Kopf des Unterkiefers (Caput mandibulae) und seine bewegliche Knorpelscheibe (Discus articularis) und die knöcherne Unterkiefergrube (Fossa mandibularis) gebildet. Für die Mahlbewegung des Kauens ist vor allem die seitliche Bewegung des Unterkiefers wichtig. Die 32 Zähne des Erwachsenen sind mit ihren Wurzeln fest im Ober- und Unterkiefer verankert und stehen leicht versetzt in der Schlussbissstellung (Okklusion). Das Kauen ist eine zyklische Bewegung mit exakt aufeinander abgestimmten Kiefer-, Wangen-, Zungen- und Hyoidbewegungen. Durch die sensomotorische Kontrolle können Bissverletzungen vermieden werden. Zur Kaumuskulatur gehören 4 Muskeln, die von Ästen des N. mandibularis versorgt werden. Der M. temporalis, der M. masseter und M. pterygoideus medialis, der auch eine Vorschubbewegung bewirkt, schließen den Kiefer. Der M. pterygoideus lateralis öffnet bei beidseitiger Kontraktion den Mund.

Den knöchernen Rahmen für den Mundboden bildet der Unterkiefer. Innen auf seiner Unterseite verlaufen längs der M. geniohyoideus und quer der M. mylohyoideus.

Die Zunge formt den Bolus und schiebt ihn zwischen die Zähne (Zungenspitze und Zungenränder schieben sich an die Alveolen) der Speichel weicht den Bolus zu einer homogeneren Masse ein. Ein vorzeitiges Abgleiten der Nahrung in den Pharynx wird durch die Anhebung des posterodorsalen Teils der Zunge und Senkung des Gaumensegels verhindert.

Am Ende der Vorbereitungsphase öffnet sich der Verschluss zwischen posterodorsaler Zunge und Gaumensegel kontrolliert und leitet damit den Übergang von der oralen in die pharyngeale Phase ein.

Während des Kauens gleiten Teile der zerkleinerten Speisen über die V-förmig abgesenkte Zungenmitte (Zungenfurche) in die Valleculae.

In dieser Phase kann man unter dem Schutz der Epiglottis mit im Oropharynx liegendem Bolus sprechen, ohne dass das Risiko einer Aspiration besteht [5]. Bei halbflüssigem Bolus kommt es früher zum Überlauf. Gleiten Teile des Bolus über die wichtige Triggerzone der Plicae pharyngoepiglotticae hinweg, wird der Schluckreflex ausgelöst.

Kleine Flüssigkeitsmengen können zwischen Zungengrund und Gaumensegel in die Valleculae gleiten, ohne einen Schluckreflex auszulösen. Mit dem Ausatmen erreichen die Duftmoleküle des Bolus über den retronasalen Weg die Regio olfactoria. So gelangen sensorische Informationen über die Rezeptoren zum Schluckzentrum und verhindern oder fördern das Schlucken.

Die Dauer der oralen Vorbereitungsphase ist individuell sehr verschieden.

\subsubsection{Orale Phase}

Die orale Phase dient dem Bolustransport vom Mund in den Oropharynx. Sie kann willkürlich gesteuert werden. Sie beginnt mit dem Einsatz der Zungenspitzenbewegung und endet mit der Auslösung des Schluckreflexes.

Durch den dichten Kontakt der Zungenspitze und der vorderen Zungenränder an den Alveolen bildet sich eine Zungenfurche für den Bolus. Die Zungenmuskulatur inseriert im Mundboden. Sie wird vom $\mathrm{N}$. hypoglossus innerviert. Man unterscheidet die Außenmuskulatur (M. genioglossus, M. hyoglossus, M. styloglossus) von der Innenmuskulatur (M. longitudinalis, M. verticalis, M. transversus). Die extrinsische Zungenmuskulatur zieht bei Kontraktion den gesamten Zungenkörper mit dem Bolus von 
vorn nach hinten. Die intrinsische Zungenmuskulatur befördert den Bolus in einer wellenförmigen Bewegung entlang des Gaumendaches nach hinten. Das Schließen des Mundes unterstützt diesen Vorgang. Zwischen Gaumensegel und Zunge kann das „Gaumensegel-Tor“ differenziert geöffnet werden, um einige Tropfen oder einen ganzen Bolus passieren zu lassen.

Die orale Phase kann beim Speichelschlucken und/oder Nachschlucken von Speisen eingeschränkt sein oder fehlen.

\subsubsection{Pharyngeale Phase}

Der Pharynx ist ein ca. 12 - $15 \mathrm{~cm}$ langer Muskelschlauch von der Schädelbasis bis zum Speiseröhreneingang. In ihm kreuzen Luftund Speiseweg. Pharynx und Ösophagus sind zwischen Schädelbasis und Zwerchfell elastisch aufgehängt. Gleichzeitig ist der Atemweg mit Hyoid und Larynx zwischen Schädelbasis und Mandibula einerseits und oberer Thoraxapertur andererseits über Muskel und Bänder elastisch vergurtet. Damit wird verständlich, dass das gesamte System, z. B. durch eine Hiatushernie, in seiner Funktion beeinflusst wird.

Der Pharynx wird in 3 Etagen unterteilt: Der Nasopharynx reicht vom Rachendach bis zum Gaumensegel und steht vorne über die Choanen mit der Nasenhöhle und über die Tuba auditiva mit dem Mittelohr in Verbindung. Der Oropharynx reicht vom Gaumensegel nach kaudal bis zur Plica pharyngoepiglottica und öffnet sich nach vorne zur Mundhöhle. Der Hypopharynx erstreckt sich vorne von der Epiglottis, seitlich über die aryepiglottische Falte zur Incisura interarytenoidea. Der Recessus piriformis liegt seitlich von der aryepiglottischen Falte und zieht bis zum oberen Ende des Ösophagus. Der Hypopharynx hat Zugang zum Larynx und zum Ösophagus.

Der Pharynxschlauch wird durch die 3 Schlundschnürer, Mm. constrictores pharyngis, und die Schlundheber, Mm. levatores pharyngis gebildet. Die Schlundschnürer bestehen aus dem M. constrictor pharyngis superior (N. glossopharyngeus, N. IX), der sich beim Schlucken als sog. Passavantscher Ringwulst dem Gaumensegel entgegenwölbt und den Nasopharynx abschließt. Ein zirkulärer Muskelzug vor den Nasengängen, der von M. Strahl als Rhinosphinkter bezeichnet wurde, ist in etwa $9 \%$ der ambulanten HNO-Patienten vorhanden [6]. Er schließt kurz vor dem Velumverschluss und öffnet kurze Zeit nach der Relaxation. Es wird angenommen, dass er eine Schutzfunktion für den Nasenrachen und damit für Nase, Nasennebenhöhlen, Tube und Mittelohr übernimmt.

Weitere Muskeln wie der M. constrictor pharyngis medius (Plexus pharyngeus, Nn. glossopharyngeus und vagus, N. IX und N. X) und der M. constrictor pharyngis inferior (N. X) unterstützen das Vorschieben des Nahrungsbolus.

In der pharyngealen Phase wird der Bolus durch die Rückwärtsbewegung der Zunge in den Hypopharynx gedrückt. Während einer ruhigen Endoskopie sieht man, wie der Bolus in der leicht eingekerbten Mitte der Zunge unter der beweglichen Uvula durch in die Vallecula gleitet. Das Zungenbein wird angehoben und bewegt sich nach ventral. Der Kehlkopf wird, unterstützt durch den M. thyrohyoideus, nach vorne oben gezogen, und es entsteht durch die Öffnung des Hypopharynx (Raumerweiterung) eine Sogwirkung. Gleichzeitig setzen die Verschlussmechanismen ein: das Gaumensegel hebt sich an die Rachenhinterwand, der Kehldeckel legt sich über den Kehlkopf, der supraglottische Raum verengt sich, die Stimmlippen und Taschenfalten schließen sich und die Aryknorpel kippen in das Lumen und legen sich über die Stimmlippen. Die Epiglottis hebt sich mit der Kontraktion des M. thyrohyoideus und senkt gleichzeitig den
Oberrand gegen die Pharynxhinterwand, um den Bolus aufzufangen. Dabei bleibt die Position des Os hyoideum relativ stabil.

Der Schluckreflex wird je nach Qualität des Berührungsreizes an den vorderen Gaumenbögen, den plicae pharyngoepiglotticae oder der postkrikoidalen Schleimhaut ausgelöst [6] und ist inter- und intraindividuell variabel, bolus- und altersabhängig. Er ist willentlich nicht beeinflussbar. Die Triggerung ist ausschließlich taktil (thermische Reize spielen physiologisch keine Rolle). Wässrige Substanzen werden als Kontinuum in Bruchteilen einer Sekunde abgeschluckt. Dickflüssige und feste Speiseanteile werden in den Valleculae gesammelt und kaskadenförmig geschluckt. Daher kann man sich mit einer durch Speise gefüllten Vallecula unterhalten, was bei Flüssigkeiten ausgeschlossen ist. Bei Säuglingen ist der harte Gaumen ohne Zähne flacher, und der Ringknorpel und der Kehlkopf stehen wesentlich weiter kranial als beim Erwachsenen. Der Kehldeckel schiebt sich hinter den weichen Gaumen, der Larynx öffnet sich direkt in den Nasopharynx; der Luftweg ist vom Speiseweg durch den höher stehenden Larynx besser getrennt und vor Aspiration geschützt. Beim Saugen können Säuglinge deshalb atmen ohne zu aspirieren, aber beim Schlucken kommt es ebenso wie beim Erwachsenen zur Schluckapnoe.

Durch die starke Rückwärtsbewegung des Zungengrundes an die Rachenhinterwand, die ebenfalls kontrahiert, wirkt die Zunge beim Weitertransport des Bolus wie ein Stempel. Gleichzeitig kommt es zur Bewegung des Zungenbeins (Os hyoideum) und des Kehlkopfes nach anterior und superior. Der M. geniohyoideus und M. mylohyoideus bewegen das Zungenbein beim Schlucken nach vorn oben. Das erweitert den Pharynx und verkürzt ihn um etwa ein Drittel.

Zum Schutz der tiefen Atemwege schließt sich der Kehlkopf in 4 Ebenen: Stimmlippenschluss, Aneinanderlegen der Taschenfalten, Annäherung der Aryknorpel an den Petiolus sowie Dorsalkippung der Epiglottis. Während des Schluckens kommt es zum reflektorischen Atemstopp.

Zum Abschluss der pharyngealen Phase kommt es zur Öffnung des oberen Ösophagussphinkters (OÖS), der erstmals von Killian beschrieben wurde. Nach manometrischen, elektromyografischen und radiologischen Ergebnissen beschränkt sich die Verschlusszone des oberen Ösophagussphinkters nicht auf den M. cricopharyngeus. Es werden kaudale Teile der Pars thyropharyngea des unteren Schlundschnürers und kraniale Abschnitte der zervikalen Ringmuskelschicht des Ösophagus funktionell mit einbezogen [7]. Ein submuköses Polster der postkrikoidalen Region hilft als kavernöses Kissen den OÖS zu verschließen.

Die Öffnung des OÖS verläuft in mehreren Schritten:

- Relaxation

- Öffnung

- Erweiterung der Öffnung

- Kollaps

- Schluss

Der OÖS relaxiert ca. 0,1 s vor der Kehlkopfhebung. Durch die Kontraktion des M. thyrohyoideus mit leichtem Kippen und Aufsteigen der Epiglottis (Elevation), gefolgt von der Vorwärtsbewegung des Larynx nach vorne oben, öffnet sich passiv die Muskelschlinge zwischen Kehlkopf und Rachenhinterwand vor Ankunft des Bolus. Die Kontraktionswelle der Konstriktormuskulatur drückt mit dem Zungenstempel den freien Rand der Epiglottis nach unten. Über ihn erreicht der Bolus wie eine Dachlawine die obere Speiseröhre. Der Bolusdruck beeinflusst die Weite der OÖS-Öffnung. Sobald der Bolus im Ösophagus ist und der Larynx 
und das Os hyoideum in ihre Ruheposition zurückgekehrt sind, schließt sich der OÖS mit einem Dauertonus [8]. Während das Gaumensegel bereits erschlafft, wird die Epiglottis durch die noch bestehende kaudale Kontraktion über dem Larynxeingang geschlossen gehalten. Sobald die Epiglottis in die Ruheposition zurückgekehrt ist, öffnet sich der Kehlkopf wieder zur Atmung, und die pharyngeale Phase ist beendet.

\subsection{5 Ösophageale Phase}

Die Speiseröhre beginnt etwa in Höhe des 6. Halswirbels und bildet einen ca. $25 \mathrm{~cm}$ langen elastischen Muskelschlauch. Die gesamte Strecke von den Schneidezähnen bis zum Mageneingang beträgt ca. $40 \mathrm{~cm}$. Der Ösophagus wird vom N. vagus über den Plexus oesophageus und vom Truncus sympathicus innerviert. Die peristaltische Bewegung wird vom N. vagus gefördert, vom Sympathikus gehemmt.

Der Ösophagus mündet spitzwinklig in den Magen (Incisura cardiaca, Hisscher Winkel).

Es ergeben sich durch den anatomischen Verlauf 3 Engen: 1. die Angustia cricoidea (durch den Ringknorpel), die engste Stelle des Ösophagus (ca. $15 \mathrm{~mm}$ Durchmesser), 2. die Angustia aortica (durch die Aorta) und 3. die Angustia diaphragmatica (durch das Zwerchfell). Veränderungen der Umgebungsstrukturen im Bereich der Engen können zu Schluckstörungen führen.

Die obere Enge wird durch den OÖS verschlossen. Die Speiseröhre besteht im obersten Viertel aus quergestreifter, im zweiten Viertel zusätzlich aus glatter und in der unteren Hälfte nur aus glatter Muskulatur. Die Muskelfasern verlaufen außen schraubenförmig (äußere Längsmuskelschicht) und innen schräg oder zirkulär (innere Ringmuskelschicht). Sie bilden eine funktionelle Einheit [9].

Bei der Peristaltik unterscheidet man eine primäre und eine sekundäre Welle. Die Beförderung des Bolus mit der reflektorischen primären Welle benötigt zwischen 4 und $40 \mathrm{~s}$ bis zum Magen. Flüssigkeiten können bei Öffnung des oberen und insuffizientem unterem Ösophagussphinkter (UÖS) in nur einer Sekunde durch die Stempelkraft von Zunge, Mundboden und Killian-Muskel in den Magen gespritzt werden. Besteht eine Hiatushernie, verliert die Speiseröhre ihre Spannung und kollabiert. Bei aufrechter Position wird der Bolustransport durch die Schwerkraft unterstützt.

Durch mechanische Reizung (Speisereste) an der Wand wird die sekundäre Welle ausgelöst. Sie fungiert als sogenannte Reinigungswelle. Die Speiseröhre besitzt eine hohe Längsspannung, sodass es grundsätzlich möglich ist, auch im Kopfstand zu schlucken.

\subsection{Zentrale Steuerung des Schluckens}

Der komplexe Steuerungsvorgang des Schluckens findet in verschiedenen Hirnstrukturen statt. Es bestehen Verbindungen in beiden Richtungen zwischen dem Großhirnkortex, den kortikobulbären Bahnen, dem Hirnstamm und der peripheren Schluckmuskulatur.

In der oberen Medulla oblongata liegen Strukturen, die als „Central Pattern Generators for Swallowing“ (CGPs) bezeichnet werden [10]. Man unterscheidet einen dorsomedialen („Dorsal Swallowing Group“, DSG) von einem ventrolateralen („Ventral Swallowing Group“, VSG) Anteil. Im dorsalen Anteil wird das räumlich-zeitliche Zusammenspiel der Schluckmuskeln koordiniert. Der Nucleus tractus solitarii ist Teil der hinteren Schluckgruppe und verarbeitet sensible Reize aus dem oropharyngolaryngealen Bereich (Temperatur, Berührung usw.). Von der ventralen Gruppe werden diese Informationen an die schluckrelevanten Hirnnervenkerne (V, VII, IX, X und XII) weitergeleitet. Die schluckrelevanten Bereiche des Kortex sind u. a. das frontoparietale Operkulum und die vordere Insel, die vor allem beim willkürlichen Schlucken aktiv ist [11].

Unabhängig von der Händigkeit besteht eine interindividuell unterschiedliche Schluckdominanz; d. h. eine Seite des Großhirns besitzt einen größeren Schluckkortex als die andere [12]. Nach zahlreichen Untersuchungen (fMRT, PET, Läsionsstudien u.a.) der letzten Jahre geht man davon aus, dass wahrscheinlich die linke Hemisphäre eher für die orale Vorbereitungsphase und die orale Phase zuständig ist, während die rechte Hemisphäre eher in der pharyngealen Phase dominiert $[13,14]$. Typisch für einseitige Läsion des dominanten Schluckkortex ist eine verzögerte Schluckreflextriggerung [15].

Die ösophageale Phase läuft weitgehend selbstständig ab, unterliegt aber auch zentralen Einflüssen, vor allem aus der Medulla oblongata [16].

Ein Zusammenfassung der verchiedenen Schluckphasen zeigt - Tab. 1.

\section{Klinik der Dysphagien}

\subsection{Pathophysiologie des Schluckens}

\subsubsection{Dysphagieeinteilung nach Lokalisation}

Je nach Lokalisation rufen Läsionen bestimmte Symptome hervor. $\diamond$ Tab. 2 zeigt eine entsprechende Zusammenstellung.

Dysphagien können prinzipiell in jedem Lebensalter auftreten. Pathophysiologische Aspekte im Kindesalter werden im Kapitel „Dysphagiediagnostik bei Kindern“ besprochen.

Störungen der präoralen Phase können als mangelndes Hungeroder Durstgefühl, unzureichende visuelle Erfassung der Nahrung

Tab. 1 Phasen des Schluckvorganges (nach [17]).

\begin{tabular}{|c|c|c|c|}
\hline Phase & Funktionsablauf & Dauer & Motorische Steuerung \\
\hline $\begin{array}{l}\text { orale Vorberei- } \\
\text { tungsphase }\end{array}$ & $\begin{array}{l}\text { Aufnahme, Zerkleinerung der Nahrung, Durchmischung mit Speichel, } \\
\text { Bolusformung durch die Zunge, Sammlung in der „Zungenschüssel“, } \\
\text { Abschluss der Mundhöhle durch Velum-Zungenbasiskontakt }\end{array}$ & $\begin{array}{l}\text { variabel (abhängig von } \\
\text { Gewohnheit, Beschaffen- } \\
\text { heit der Nahrung) }\end{array}$ & willkürlich \\
\hline $\begin{array}{l}\text { orale Transport- } \\
\text { phase }\end{array}$ & $\begin{array}{l}\text { Beförderung des Bolus in die hintere Mundhöhle durch Anhebung und } \\
\text { Rückwärtsbewegung der Zunge }\end{array}$ & $\leq 1 \mathrm{~s}$ & willkürlich \\
\hline $\begin{array}{l}\text { pharyngeale } \\
\text { Phase }\end{array}$ & $\begin{array}{l}\text { Auslösung des Schluckreflexes, Transport des Bolus im Pharynx durch } \\
\text { Stempeldruck der Zunge und die Pharynxperistaltik, Verschluss des } \\
\text { Nasopharynx, Anhebung, Vorwärtsbewegung und Verschluss des Larynx, } \\
\text { Öffnung des oberen Ösophagussphinkters (OÖS) }\end{array}$ & $\leq 1 \mathrm{~s}$ & reflektorisch \\
\hline $\begin{array}{l}\text { ösophageale } \\
\text { Phase }\end{array}$ & $\begin{array}{l}\text { Transport des Bolus durch den Ösophagus durch peristaltische Wellen, } \\
\text { Öffnung des unteren Ösophagussphinkters (UÖS) }\end{array}$ & $4-40 s$ & reflektorisch \\
\hline
\end{tabular}


Tab. 2 Symptome oropharyngealer und ösophagealer Dysphagie (modifiziert nach [18]).

\begin{tabular}{|c|c|}
\hline $\begin{array}{l}\text { oropharyn- } \\
\text { geale } \\
\text { Dysphagie }\end{array}$ & $\begin{array}{l}\text { Drooling } \\
\text { mangelnde orale Boluskontrolle und Transport } \\
\text { Residuen im Cavum oris } \\
\text { Schwierigkeiten, den Schluckvorgang einzuleiten } \\
\text { nasale Penetration } \\
\text { Steckenbleiben von Nahrung im Hals, Husten, Räuspern, } \\
\text { Regurgitation } \\
\text { Änderung des Stimmklangs während oder direkt nach } \\
\text { dem Essen, Trinken oder der Medikamenteneinnahme } \\
\text { verlängerte Essdauer } \\
\text { Notwendigkeit einer Änderung der Nahrungskonsistenz } \\
\text { verstärkte Verschleimung } \\
\text { gehäufte Infekte, Pneumonien } \\
\text { Gewichtsabnahme }\end{array}$ \\
\hline $\begin{array}{l}\text { ösophageale } \\
\text { Dysphagie }\end{array}$ & $\begin{array}{l}\text { Halsschmerzen, thorakale Schmerzen, Brennen in der } \\
\text { Herzregion } \\
\text { Globusgefühl (physiologisch bei gefülltem Magen, bei } \\
\text { Überlauf in die Speiseröhre und bei dem resultierenden } \\
\text { Rückfluss in die obere Speiseröhre) } \\
\text { Regurgitation von Speisen und Tabletten } \\
\text { rezidivierende Pneumonien oder Exazerbation von } \\
\text { Asthma und COPD } \\
\text { Hustenattacken beim Hinlegen nach dem Essen } \\
\text { „Feststecken“ von Nahrung im Hals, hinter dem Brustbein } \\
\text { Gewichtsabnahme }\end{array}$ \\
\hline
\end{tabular}

oder manuelle Unzulänglichkeiten bei der mundgerechten Zerkleinerung der Nahrung beobachtet werden [19]. Diese Probleme werden hier nicht näher beschrieben.

\subsubsection{Strukturveränderungen}

In ruhiger Respiration beurteilt man Schleimhautbeschaffenheit, Symmetrie der Strukturen, Defekte, Narben, Ödeme und Vorwölbungen der Rachenhinterwand. Bei Patienten nach Kopf-/ Hals-Tumortherapie können Veränderungen der Schleimhaut das Schluckvermögen beeinträchtigen. Mukosaveränderungen sind Rötung, Schwellung, Ulzera, später auch Atrophie, narbige Veränderungen und Trockenheit ( $\bullet$ Abb. 1). Eine Verplumpung der Epiglottis schränkt die normale Dorsalflexion während des Schluckens ein und ist damit ein Aspirationsrisiko [20].

Eine Refluxsymptomatik gefährdet Patienten mit Dysphagie besonders, da Magensaft aspiriert werden kann. Refluxzeichen sind Ödeme der Larynxstrukturen, ein Pseudosulkus der Stimmlippen durch ein infraglottisches Ödem von der vorderen Kommissur bis zur Hinterwand des Larynx, eine Verdickung der Schleimhaut der Interarytenoidregion, eine verstärkte Gefäßzeichnung, Ulzera und Granulationen von Aryknorpeln und Stimmlippen sowie zäher Schleim in Hypopharynx und Larynx $[21,22]$.

\subsubsection{Paresen}

Zentrale und periphere Paresen können einen ungenügenden Glottisschluss während des Schluckens verursachen. In einer Studie mit 2650 Patienten mit Dysphagie wiesen 4,5\% der Patienten Stimmlippenparesen auf. Die Aspirationsrate betrug bei einer rechtsseitigen Parese $37 \%$, bei einer linksseitigen $42 \%$ und bei beidseitiger Parese $50 \%$. Das Aspirationsrisiko, insbesondere bei Flüssigkeiten, war 2,5-fach höher als für Patienten ohne Parese [23]. Aufgrund der über die Stimmlippenbeweglichkeit hinausgehenden motorischen und sensiblen Funktionen erhöht sich bei Lähmung von N. glossopharyngeus, N. vagus und N. hypoglossus das Aspirationsrisiko nochmals erheblich ( $\bullet$ Abb. 2, 3).

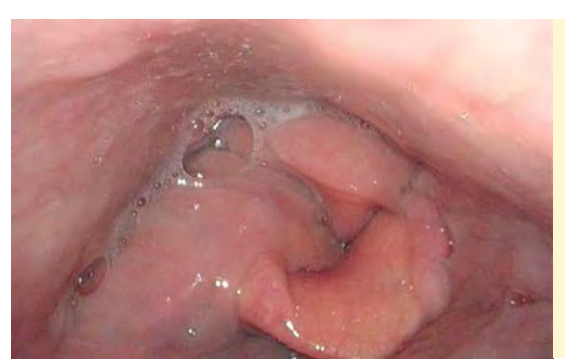

Abb. 1 Transnasale Endoskopie 8 Tage nach Cricohyoidoepiglottopexie, postoperative Schwellung ohne erkennbares Lumen, Aspiration und Speichelretention.

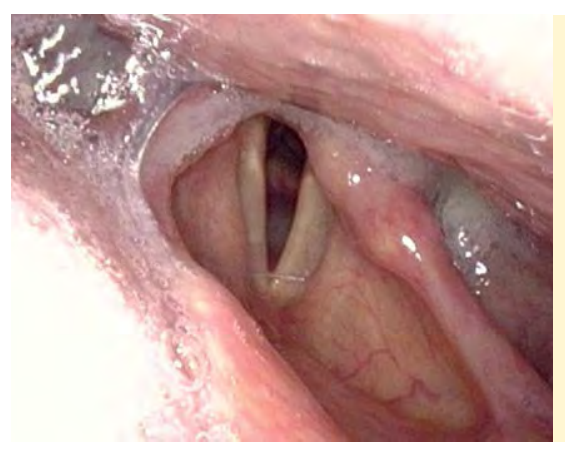

Abb. 2 72-jährige Patientin mit Zungengrundstruma und linksseitiger Stimmlippenparese. Sichtbare Speichelretentionen im Recessus piriformis, Penetration, stille Aspiration.

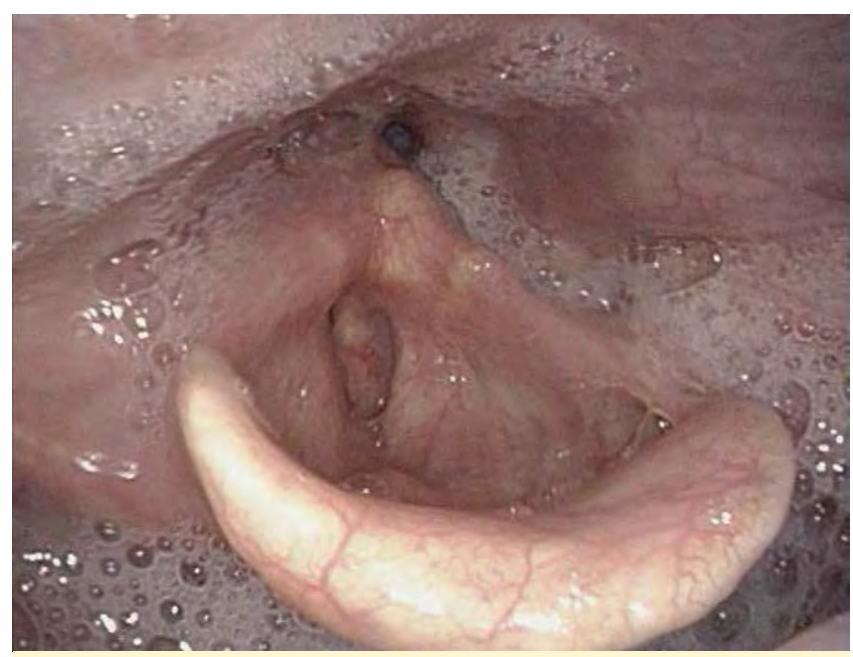

Abb. 3 70-jährige Patientin mit beidseitiger Stimmlippenparese nach totaler Thyroidektomie, Neck dissection, Radiatio. Chronische Radiodermatitis. Larynx durch Vernarbung deutlich nach ventral verlagert. Eingeschränkte supraglottische Sensibilität. Der OÖS ist offen, Schaumspeichelpooling. Penetration.

\subsubsection{Hyperkinesen}

Unwillkürliche rhythmische Bewegungen des Gaumensegels, der Rachenhinterwand und des Kehlkopfs mit Frequenzen von 1 - 3 /s mit schnellerer Adduktions- und langsamerer Abduktionskomponente sind besonders häufig bei Patienten mit Kleinhirn- und/oder Hirnstammläsionen sowohl in Ruhe (sogar im Schlaf) als auch bei willkürlichen und reflektorischen Bewegungen vorhanden. Sie sind bisweilen schon von außen an nystagmusartigen Bewegungen des Kehlkopfs und/oder des Mundbodens/der Halsmuskulatur erkennbar. Da der Glottisschluss zwar möglich, aber zeitlich nicht mit den Schluckphasen synchronisiert ist, kann es durch den funktionell insuffizienten Glottisschluss zur Aspiration kommen [24].

\subsubsection{Sensibilitätsstörungen}

Hinweise auf eine Sensibilitätsstörung des Kehlkopfs sind Überlauf von Speichel oder Speisebrei in die Glottis mit und ohne 
Tab. 3 Übersicht zur Nomenklatur von Symptomen bei Schluckstörungen.

\begin{tabular}{|ll}
\hline Drooling & Herauslaufen des Bolus oder Speichels aus dem Mund \\
\hline Regurgitation & Hochwürgen von Nahrung \\
\hline Leaking & $\begin{array}{l}\text { Herabgleiten des Bolus in den Rachen vor Auslösung des Schluckreflexes aufgrund gestörter Oralmotorik und/oder } \\
\text { Sensibilität mit beeinträchtigter Boluskontrolle }\end{array}$ \\
\hline $\begin{array}{l}\text { Pooling } \\
\text { Residuen oder Retentionen }\end{array}$ & $\begin{array}{l}\text { Ansammlung von Substanzen im Hypopharynx aufgrund fehlender oder verspäteter Auslösung des Schluckreflexes } \\
\text { Valleculae, den Recessus piriformes und in der Postkrikoidregion }\end{array}$ \\
\hline Penetration & Eintreten von Speichel, Sekret oder Bolusanteilen in den Kehlkopf ohne Durchtritt durch die Stimmlippenebene \\
\hline Aspiration & Durchtritt von Speichel, Sekret oder Bolusanteilen durch die Stimmlippenebene \\
\hline nasale Regurgitation/Penetration & Übertritt von Speichel, Sekret oder Bolusanteilen in den Nasopharynx, die Nasenhaupthöhle oder aus der Nase heraus
\end{tabular}

Tab. 3a Altersbedingte Veränderungen in den einzelnen Schluckphasen, die zu einer Presbydysphagie führen können (modifiziert nach [19]).

\section{Orale Phase}

- Schmeck-/Riechstörung

- Verminderung der oralen Sensibilität

- Einschränkung der Kaufunktion

- reduzierte Speichelproduktion

- Einschränkung der orofazialen Motorik

- Abnahme der tonischen Zungenkraft

- Störung der Phasenkoordination

- Verzögerte Schluckreflextriggerung als

Einleitung der pharyngealen Phase

\section{Pharyngeale Phase}

- erweiterte Pharynxstrukturen

- verringerte Pharynxstabilität

- stärkere Larynxelevation

- verspätet einsetzende Hyoidverlagerung

- vermehrtes Auftreten einer krikopharyngealen Barriere

- reduzierter Ruhetonus des oberen Ösophagussphinkters

- reduzierte Öffnung des Ösophagussphinkters

- vermehrter Kraftaufwand für den Bolustransport

- Reduktion der maximalen Krafterzeugung

- verlängerte pharyngeale Transitzeit

- Sensibilitätsverlust der Pharynxmukosa

\section{Ösophageale Phase \\ - erweitertes Ösophaguslumen \\ - rigide Ösophaguswand \\ - Abnahme der Ganglienzellen des Plexus myentericus \\ - reduzierte Ösophagussensibilität \\ - reduzierte oder ausbleibende sekundäre Ösophagusperistaltik \\ - reduzierte ösophageale Kontraktionskraft \\ - eingeschränkte Funktion des unteren \\ Ösophagussphinkters (fraglich)}

Hustenreflex, Reizerscheinungen in Glottis und Subglottis in Form von Rötung und Gefäßinjektion sowie gurgelnde und raue Stimmqualität ( $\bullet$ Tab. 3).

Speichel und Sekretansammlungen an den Hypopharynxwänden, in den Valleculae, den Recessus piriformes und in der Postkrikoidregion sind immer Zeichen einer Schluckstörung. Sie können auch im Aditus laryngis, in der Glottis und in der Subglottis bis zur Trachea beobachtet werden. Lösen sie keinen Hustenreflex aus, handelt es sich um eine stille Aspiration (,„silent aspiration“), die auf längere Frist lebensbedrohlich sein kann - umso mehr, wenn der Patient auch willkürlich nicht abhusten kann.

Die Erfassung von Speichel- und Sekretansammlungen ist wichtig für die Einschätzung der Gefährdung eines Patienten mit Dysphagie [25,26]. Hier ist die endoskopische Untersuchung eindeutig im Vorteil, da diese Substanzen radiologisch nicht visualisierbar sind [27].

Bei fehlender Sensibilität kommt es vor Auslösung der reflektorischen pharyngealen Phase zu einem vorzeitigen, unkontrollierbaren Eintritt von Substanzen in den Pharynx, einer Ansammlung von Substanzen im Hypopharynx, zu einer prädeglutitiven Penetration/Aspiration. $₫$ Tab. 3 fasst die Begriffe zur Beschreibung von Schluckstörungen zusammen.

Eine verzögerte Schluckreflexauslösung ist charakterisiert durch verzögerte Anterior- und Aufwärtsbewegung der Aryknorpel $[28,29]$, verzögerte Anhebung und Rückführung des Zungengrundes. Beim Gleiten des Bolus in die Valleculae bzw. die Recessus piriformes hebt sich der Kehlkopf verzögert an und die Epiglottis neigt sich nicht dorsalwärts.

Die Dorsalflektion der Epiglottis während der pharyngealen Phase ist ungenügend und verkürzt, was sich indirekt aus der Dauer des „white out“ (die durch Berührung mit der Schleimhaut beim Larynxverschluss bedingte Überblendung der Kamera) bei der fiberoptischen Schluckuntersuchung ableiten lässt $[29,30]$.

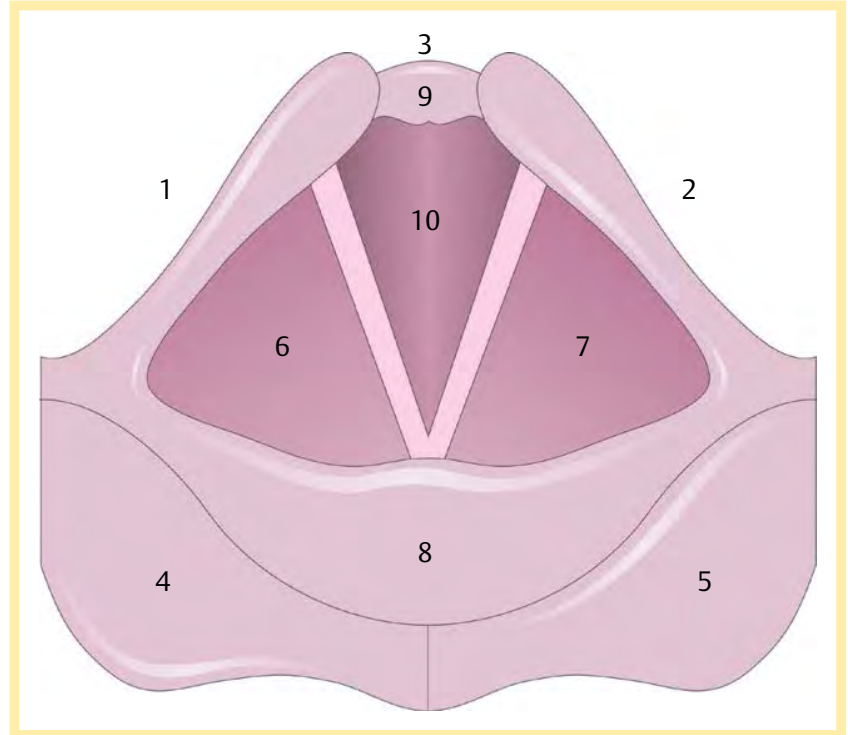

Abb. 4 Kehlkopfschema mit Lokalisation von Residuen (1-5), Penetration (6-9), Aspiration (10) [31].

Nach Ablauf der pharyngealen Phase können transnasal oder transoral die klassischen Zeichen für eine Dysphagie [31] beobachtet werden ( $\bullet$ Abb. 4).

1. Residuen von Substanzen an den Pharynxwänden, in den Valleculae und den Recessus piriformes, in der Postkrikoidregion mit/ohne Versuch des Rachenreinigens

2. Penetration von Substanzen in den Larynxeingang: an die laryngeale Fläche der Epiglottis, über die aryepiglottische Falten, über die Interarytenoidregion ohne/mit Auslösung eines Hustenreflexes

3. Aspiration von Substanzen in die Glottis, in die subglottische Region ohne/mit Auslösung eines Hustenreflexes 


\subsubsection{Krikopharyngeale Funktionsstörungen}

Die krikopharyngealen Funktionsstörungen durch Sphinkterhypertrophie mit funktioneller Stenose sind sekundär meist Folge einer Einschränkung der Exkursion von Zungenbein und Larynx. Primär sind sie Folge einer Koordinationsstörung zwischen pharyngealer Propulsion und fehlender ösophagealer Relaxation, z. B. nach Hirnstammläsionen, beim idiopathischen Parkinsonsyndrom oder bei Myositiden. Krikopharyngeale Funktionsstörungen führen zur Retention von Speise im krikopharyngealen Übergang mit der Gefahr einer insbesondere bei Kindern und alten Menschen postdeglutitiven, potenziell lebensbedrohlichen Aspiration. Eine krikopharyngeale Funktionsstörung bei Patienten nach einer Laryngektomie wird durch eine adjuvante Radiatio oft noch verstärkt und erschwert nicht nur das Schlucken, sondern auch die Phonation über die Stimmprothese durch eine verminderte Luftpassage mit konsekutiv mangelnder Schwingung des pharyngo-ösophagealen Segments [32].

\subsubsection{Presbydysphagie}

Die altersbedingten, physiologischen Veränderungen im Schluckvorgang werden als Presbyphagie bezeichnet [19, 33, 34]. Die anatomischen und physiologischen Prozesse für einen geordneten Schluckvorgang, d. h. eine entsprechende Schluckkoordination und die sensomotorische Integration von unterschiedlichen anatomischen Funktionseinheiten, Muskeln, Nerven und Hirnzentren, verändern sich beim „Älterwerden“, speziell im „4. Lebensalter“, der Demenz. Die Verringerung von Geschmacks- und Geruchssinn, zerebral-degenerative Prozesse und Veränderungen in Qualität und Quantität neuro-muskulärer Koordinationsprozesse beeinflussen die Schluckleistung meistens negativ und können alle 3 Schluckphasen betreffen. Die Schluckeffektivität lässt nach und eine Adaptation bei der Nahrungsaufnahme wird notwendig [19]. Die ösophageale Phase verlängert sich mit zunehmendem Alter. Diese presbyphagischen Veränderungen werden normalerweise kompensiert [35]. Erst wenn die Kompensationsstrategien nicht mehr ausreichen, resultiert eine Dysphagie, für die der Begriff „Presbydysphagie“ steht.

Bei einer pathologischen Erweiterung des oberen Ösophagus wird die Speise am Ende der pharyngealen Phase, wenn der Druck nachlässt, wieder in den Pharynx zurückgeworfen. Damit verdoppelt sich die Zeit für die Boluspassage, ohne dass sich die Epiglottis aufrichtet, von 0,7 auf 1,4 s [36]. Dem Patienten wird ein Schluckproblem bewusst, wenn die Boluspassage auf das 3bis 4-fache verlängert ist [37].

Patienten mit zunehmendem Alter haben darüber hinaus vermehrt Begleitkrankheiten, die additiv das Schlucken negativ beeinflussen und ebenfalls eine Dysphagie bedingen können. Hiervon sollte die Presbyphagie abgegrenzt werden, eine eindeutige Zuordnung dürfte jedoch häufig schwierig sein [18]. O Tab. 3a zeigt, welche altersbedingten Veränderungen in den einzelnen Schluckphasen zu einer Presbydysphagie führen können.

\subsection{Erkrankungen des Kopf-Hals-Gebietes mit Dysphagie}

Schluckstörungen sind grundsätzlich lebensbedrohlich. Da die Folgen nicht akut einsetzen, werden gerade die chronischen Beschwerden bei Dysphagie meist noch unterschätzt.

Erkrankungen im oberen Aerodigestivtrakt, die zerebrale, neurologische, traumatologische Ursachen haben sowie Folgen von Tumorbehandlung durch strukturelle Defekte, Narben, Schädi- gung von Hirnnerven und Bestrahlung [17] sind, können Schluckstörungen verursachen.

Eine Hirnverletzung oder Hirnerkrankung ist im Akutstadium häufig mit Bewusstseinsverlust und Störungen von Atmung und Kreislauf verbunden. Die Aufrechterhaltung der vitalen Funktionen durch Langzeitintubation und Sondenernährung kann zusätzlich zur ursächlichen Erkrankung zu Folgeschäden im Pharynx- und Larynxbereich führen. Zudem haben Patienten z. B. mit einem schweren Schädel-Hirn-Trauma neben der Hirnverletzung häufig weitere Verletzungen wie periphere Nervenläsionen, Kieferfrakturen und Weichteilverletzungen. Die Folgen globaler neuropsychologischer Störungen, wie Minderung von Aufmerksamkeit und Antrieb, können auch langfristig den Schluckablauf beeinträchtigen.

\subsubsection{Orale und pharyngeale Erkrankungen}

3.2.1.1 Störungen der präoralen Phase. Durch angeborene oder erworbene Riechstörungen kann bereits die präorale Phase gestört sein, zu der das olfaktorisch-gustatorische Antizipieren der Nahrung und die Anbahnung des Schluck(reflex)regelkreises mit potentiellem „Leerschlucken“ oder „Spontansalivation“ gehört.

3.2.1.2 Störungen der oralen Phase. Zu den kongenitalen Erkrankungen, die vorrangig die orale Phase beeinträchtigen können, gehören angeborene Fehlbildungen, wie isolierte oder kombinierte Lippen-Kiefer-Gaumenspalten (LKGS), angeborene Lähmungen der Gesichtsmuskulatur und morphologische Malformationen, z. B. bei speziellen genetischen Syndromen ( $\bullet$ Abb. 5).

Die Kieferöffnung zur Nahrungsaufnahme und die Kaubewegung des Unterkiefers können sowohl prä- und postoperativ bei Tumorerkrankungen aber auch durch strahlungsbedingte Gewebeatrophien eingeschränkt sein (Trismus). Insbesondere Tumoren in der Mundhöhle, speziell der Zunge ( $\boldsymbol{\otimes} \mathbf{A b b} \mathbf{b}$ ), führen zu großen Problemen bei der Bolusbildung, die sich durch Teilresektion der Zunge und des Mundbodens noch verstärken ( $\odot$ Abb. 7). Auch parapharyngeale Tumoren können eine Ursache für Dysphagie sein ( $\boldsymbol{O} \mathbf{A b b}$. 8). Darüber hinaus verursacht Leaking ggf. einen gestörten Bolustransport und eine prädeglutitive Aspiration.

Weitere erworbene Störungen stellen Vernarbungen nach Operationen, Frakturen, unterschiedlichste Verletzungen durch exogene Traumen wie Pfählungen, Verbrennungen oder Schnitt-

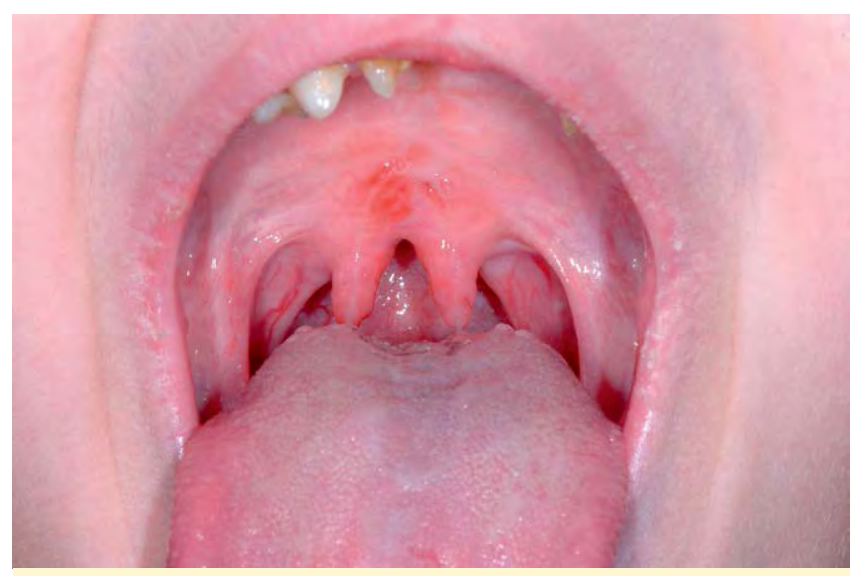

Abb. 5 16-jähriger Patient mit Johanson-Blizzard-Syndrom. Inkomplett versorgte Lippen-/Kiefer-/Gaumenspalte und velopharyngeale Insuffizienz mit Übertritt von Speisen und Flüssigkeiten in die Nase. 
wunden dar ( $\bullet$ Abb. 9). Bei partiellen Lähmungen der buccofazialen oder Zungenmuskulatur, myofunktionellen Störungen oder durch postoperative Narbenbildungen an der Zunge können die Nahrungsaufnahme, die Bolusbildung (wegen Beeinträchtigung der Lateralbewegung, der Schüsselbildung, des Stempeldrucks der Zunge) und der Bolustransport gestört sein. Selbst kraniomandibuläre Dysfunktionen behindern die orale Phase, insbesondere die Kieferöffnung und die Mahlbewegung des Unterkiefers.

Das Einspeicheln der Nahrung bedarf einer funktionierenden Salivation. Die Xerostomie kann durch Medikamente verursacht sein, oder es besteht $z$. B. eine Störung aus dem rheumatischen Formenkreis (M. Sjögren). Hauptursache für eine Hyposalivation ist jedoch die strahlenbedingte Zerstörung des Speicheldrüsengewebes.

Einschränkungen der Velummotilität und des velopharyngealen Verschlusses beeinträchtigen erheblich die Lebensqualität. Sie sind bedingt durch kongenitale (LKGS) sowie traumatisch oder iatrogen bedingte Defekte bzw. durch Narbenstränge nach ausgedehnten Resektionen von Oropharynxtumoren. Symptome sind Hyperrhinophonie und Rhinolalie, eingeschränkte Schluckreflextriggerung, gestörter bzw. fehlgeleiteter Bolustransport

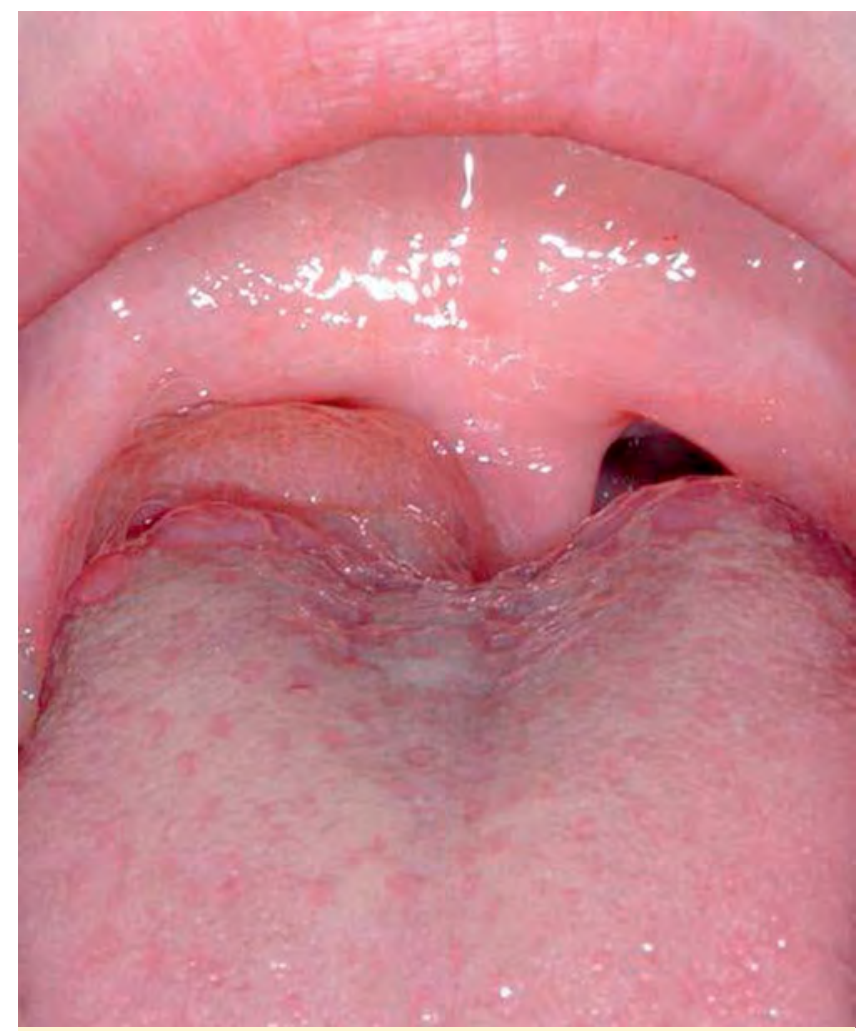

Abb. 6 Lymphangiom rechter Zungengrund. Anamnestisch zunehmend, Störung der oropharyngealen Phase. oder vorzeitiges Abgleiten des Bolus in den Pharynx ohne koordinierten Schluckablauf.

\subsubsection{Störungen der pharyngealen Phase}

3.2.2.1 Raumforderungen. Alle pharyngealen Tumoren beeinträchtigen ab einer gewissen Größe das Schlucken ( $\bullet$ Abb. 2,10). In der Regel sind es Plattenepithelkarzinome, die häufig erst spät durch eine Metastasierung eine Dysphagie hervorrufen. Für die Schwere der Dysphagie sind die Größe des Tumors, die infiltrierten Strukturen von Hypopharynx und Larynx wie auch der Funktionsverlust maßgeblich. Speziell Hypopharynxtumoren bleiben lange Zeit unentdeckt und können sich, da sie keine anatomischen Barrieren haben, ungehindert ausbreiten. Tumoren die den Larynx und Hypopharynx befallen, können zu Kombina-

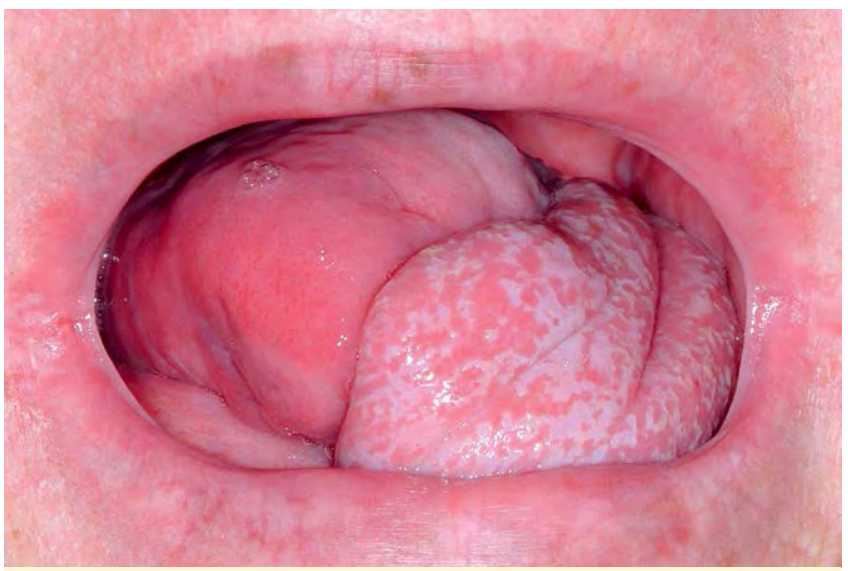

Abb. 7 53-jährige Patientin mit linksbetonter Zungenrekonstruktion nach Hemiglossektomie. Kombinierter Radialislappen mit neurovaskulärem, infrahyoidalem, myofaszialem Lappen nach Remmert. Partiell gestörter Bolustransport der oralen Phase.

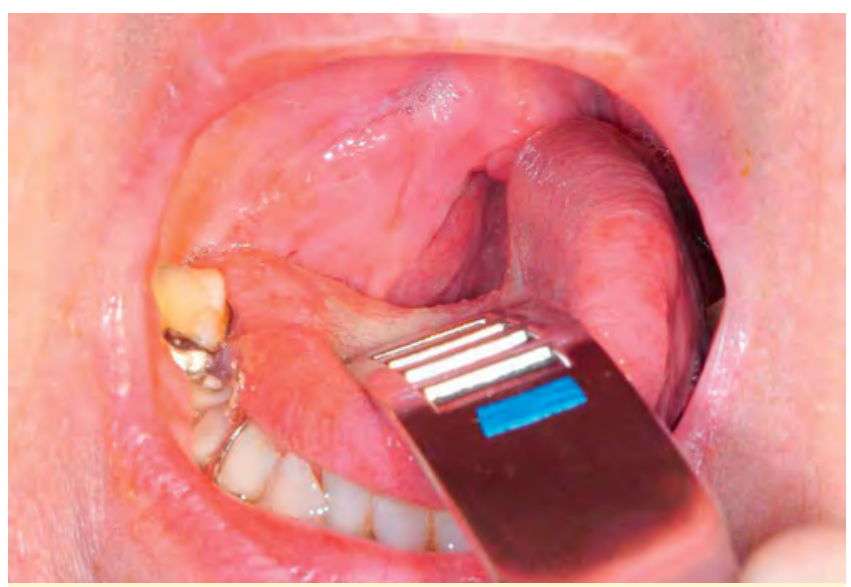

Abb. 8 69-jährige Patientin mit rechtsseitigem parapharyngealem Neurofibrom und zunehmender Dysphagie. Uvulaverdrängung nach links, Schluckstörung oropharyngeale Phase.
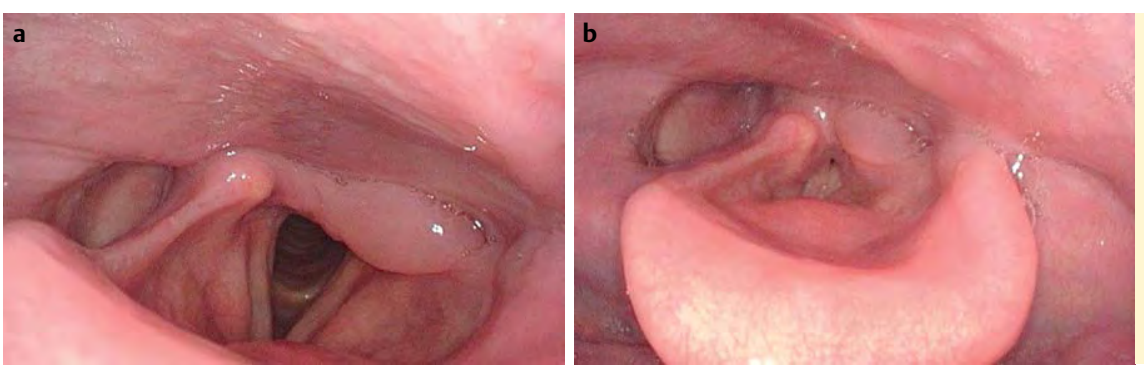

Abb. 9 46-jähriger Patient mit persistierender Dysphagie nach Schussverletzung in den linken Hals mit Perforation des Pharynx, Notfallversorgung vor 3 Monaten. Keine Öffnung des Recessus piriformis. Persistierende Schwellung der linken Aryregion und aryepiglottischen Falte, Speichelretention. Respiration a, Phonation $\mathbf{b}$. 

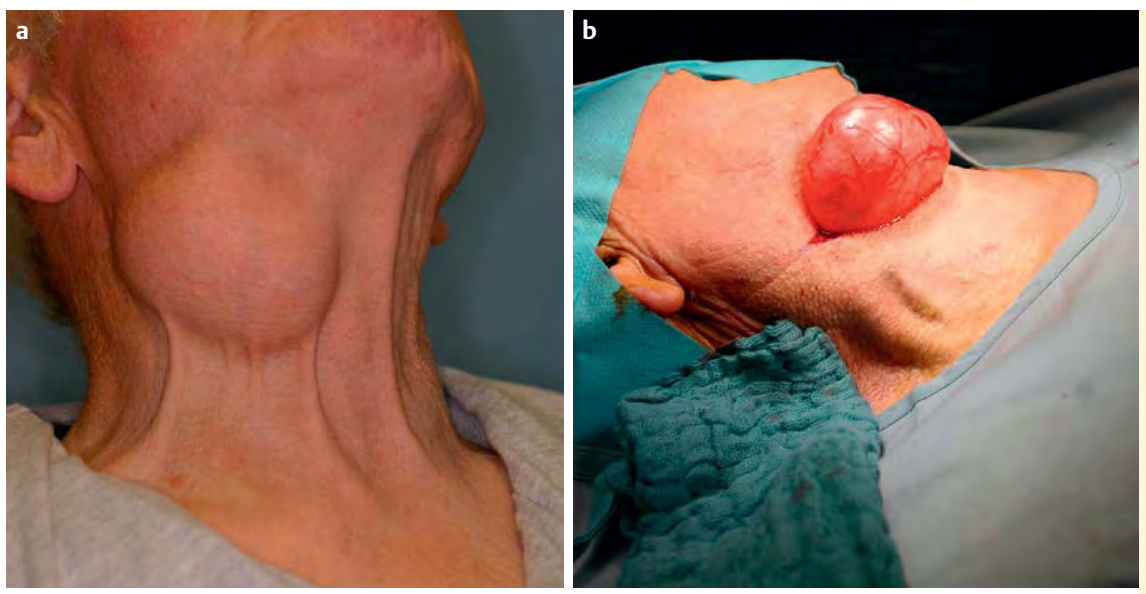

Abb. 10 73-jähriger Patient mit großer rechtsseitiger innerer und äußerer Laryngozele. Zunehmende Dysphagie und kloßige Sprache. a deutlich hervortretende Raumforderung rechter Hals. b intraoperative Situation. c Präoperatives endoskopisches Bild. LC-Laryngozele, EPI-Epiglottis, PH-Pharynxhinterwand. d Horizontale Computertomografie des Halses mit der großen rechtsseitigen Laryngozele.
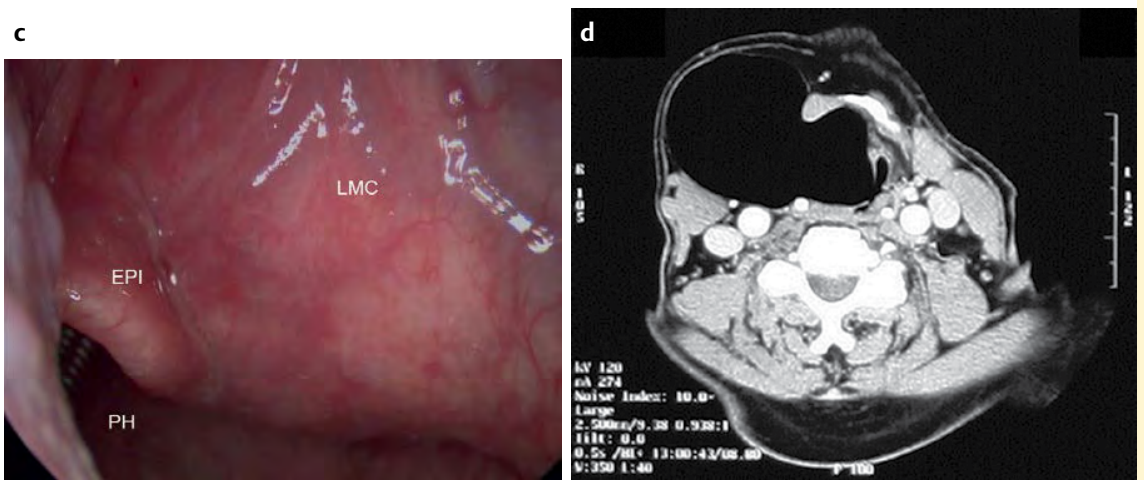

tionen von Dysphagie und Dysphonie führen. Postoperativ sind bei diesen Tumoren Substanzverlust und eine veränderte Anatomie die Regel. Dysphagien entstehen z. B. durch ein ungehindertes Abgleiten von Substanz in die Glottis. Schädigungen des $\mathrm{N}$. laryngeus superior im Rahmen von Teilresektionen oder nach Strumektomie führen $\mathrm{zu}$ einem verminderten Schutzreflex (Husten) und dadurch ggf. zu stillen Aspirationen. Gerade nach Resektion von Teilen der Epiglottis bzw. der aryepiglottischen Falte muss das Schlucken neu erlernt werden. Diesen meist passageren Schluckstörungen müssen die chronischen Schluckstörungen durch Narbenbildung und ödematose Schwellungen, z. B. nach Radiatio gegenübergestellt werden.

Nach Laryngektomie kann der Bolustransport behindert werden, bspw. durch narbige Strikturen mit Lumenverengungen des Neopharynx und oberen Ösophagus im pharyngo-ösophagealen Segment ( $\bullet$ Abb. 11). Eine Aspiration kann über eine tracheoösophageale Fistel mit Stimmventil entstehen.

3.2.2.2 Radiochemotherapie und Dysphagie. Die Dysphagie ist eine relevante Nebenwirkung der Strahlentherapie bei Kopf-Hals-Karzinomen und kann potentiell dosislimitierend sein [38]. Die Schluckfunktion kann durch Ödeme, Neuropathien und Fibrosen gestört sein. Akute Mukositiden und Ödeme beeinträchtigen die Schluckfunktion während der Bestrahlung. Sie bessern sich jedoch bei den meisten Patienten deutlich in den Monaten nach der Strahlenbehandlung oder Radiochemotherapie. Im Gegensatz dazu entwickeln sich Neuropathie und Fibrosen der oralen, laryngealen und pharyngealen Muskulatur lange nach Beendigung der Strahlentherapie. Nach Nguyen [39] treten Dysphagien häufiger bei der Radiochemotherapie im Vergleich zur alleinigen Radiatio auf. Eine Aspiration trat grundsätzlich in beiden Gruppen auf. Frowen et al. [40, 41] berichten über die Langzeitergebnisse der Dysphagie bei Patienten mit KopfHals-Tumoren, die 6 Monate bzw. 5 Jahre nach der Polychemora-
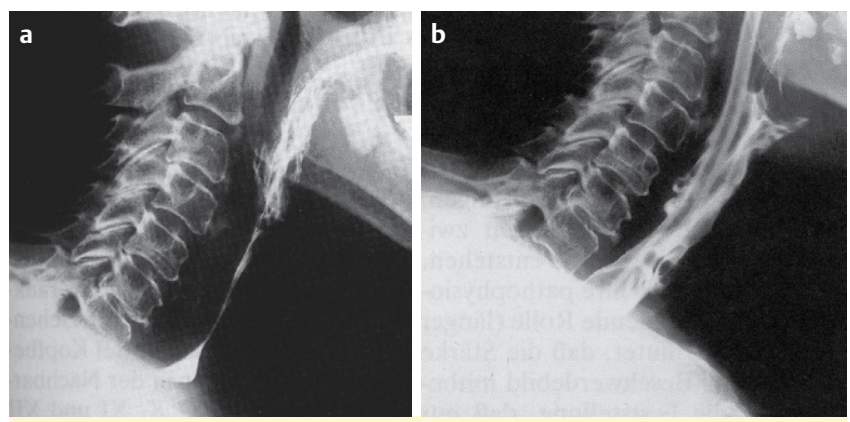

Abb. 11 Larynxstenose nach Laryngektomie und Anschlussbestrahlung. a Vor OP. b Haut-Platysma-Faszienlappen [279].

diotherapie bzgl. ihrer Schluckfunktion untersucht wurden. Ausgeprägte Schluckstörungen bestanden in den ersten 3 Monaten nach Radiochemotherapie. Die Dysphagie normalisierte sich nach 6 Monaten. Als Faktoren für eine schlechtere postoperative Schluckfunktion wurden ein ehemaliger schwerer Alkoholkonsum, eine fortgeschrittene Tumorgröße (T4), Tumorlokalisation im Hypopharynx, eine bilaterale Bestrahlung des Pharynx sowie eine ländliche Herkunft genannt. Als häufigste Prädiktoren der Dysphagie wurden die T-Klassifikation, gefolgt von der Alkoholanamnese und der Bestrahlungsart aufgeführt. Nach 5 Jahren waren $74 \%$ der behandelten Patienten zu ihrem prätherapeutischen Funktionszustand zurückgekehrt. Umgekehrt wiesen $24 \%$ eine signifikante Verschlechterung der Schluckfunktion auch noch nach 5 Jahren auf. Van den Berg et al. [42] beschrieben bei $75 \%$ der Patienten mit Kopf-Hals-Tumoren eine Schluckstörung nach Polychemoradiotherapie. Bei $57 \%$ konnten pathologische Befunde in der Videofluoroskopie beobachtet werden. Nach Gourin et al. [43] sind eine prätherapeutische Dysphagie, Radiochemotherapie und „Salvage-Chirurgie“ signifikante Prädiktoren für eine langfristige Dysphagie bei älteren Patienten mit 

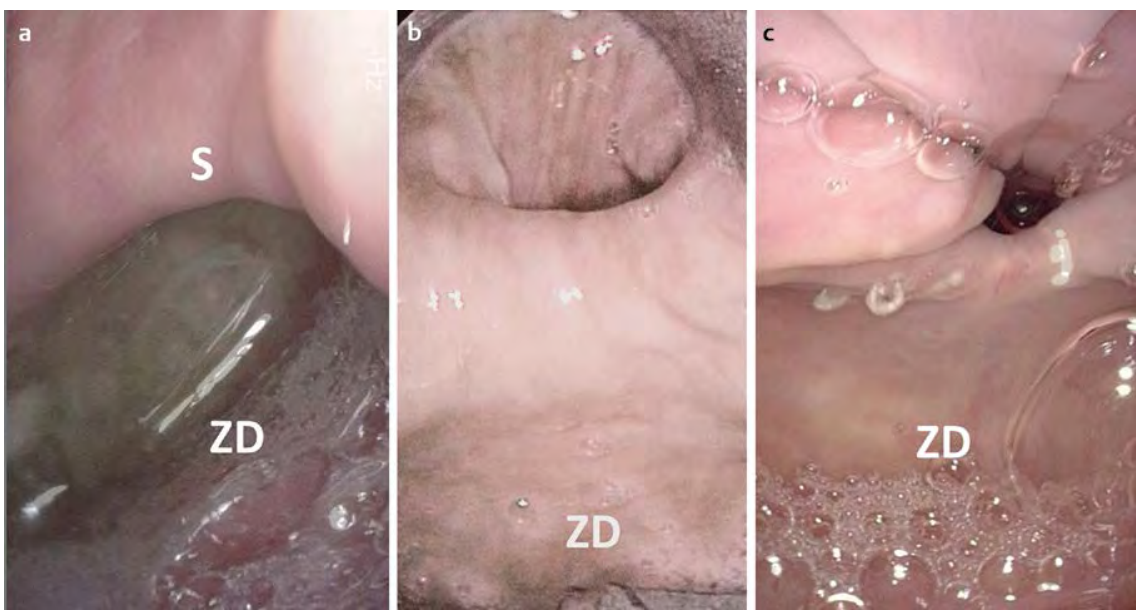

Abb. 12 a Exponierte Schwelle (S) des ZenkerDivertikels (ZD) während flexibler Endoskopie. b Intraoperativ mit Divertikuloskop. c 6 Monate nach transoral endoskopischer Schwellendurchtrennung mit Stapler, diskrete Restschwelle, Patient beschwerdefrei.

einem Kehlkopfkarzinom. Andererseits können eine langfristige Dysphagie, das Vorliegen einer PEG und/oder Tracheotomie, Gewichtsverlust, Obstruktion der Atemwege und Pneumonie mit einer geringeren Überlebensrate assoziiert sein. Vor allem in Verbindung mit einer Pneumonie ergab sich das höchste Mortalitätsrisiko bezogen auf eine 5-Jahresüberlebenszeit.

3.2.2.3 Zenker-Divertikel. $\mathrm{Zu}$ den organisch bedingten Störungen der pharyngealen Phase zählt auch das Zenker-Divertikel. Hierbei entwickelt sich der Divertikelsack als Schleimhautausstülpung (falsches Divertikel) zwischen dem unteren horizontalen und dem oberen schrägverlaufenden Anteil des M. cricopharyngeus ( $\bullet$ Abb. 12). Dieser Locus minoris resistentiae wird auch Killian'sches Dreieck genannt. Das Divertikel geht meist mit einem Globusgefühl sowie Regurgitationen von Residuen aus dem Divertikel einher. Komplikationen des Zenker-Divertikels sind Aspirationspneumonien, Perforationen und Blutungen.

\subsubsection{Störungen der ösophagealen Phase}

Entzündungen der Speiseröhre, wie bspw. eine eosinophile Ösophagitis oder Entzündungen durch Reflux, Soor bzw. Viren (Herpes oesophagi), behindern zwar nicht den Bolustransport, bereiten aber unter Umständen starke Schmerzen beim Schlucken (o Abb. 13, 14).

Neben Motilitätsstörungen des Ösophagus, die etwa durch Ösophagusspasmen, Achalasie, Barrett-Ösophagus, Sklerodermie, Vaskulitiden oder Kollagenosen ausgelöst sind, können vor allem Divertikel und Stenosen diese Phase stark beeinträchtigen.

$\mathrm{Zu}$ Tumoren des Ösophagus zählen das Adeno- und das Plattenepithelkarzinom. Dysphagien sind dann Folge des Tumors, der chirurgischen Therapie und/oder der Radio- oder Radiochemotherapie.

\subsection{Zervikogene Dysphagie}

Zervikogene Schluckstörungen können in 3 verschiedene Gruppen unterteilt werden:

1. Funktionelle Störungen der Halswirbelsäule (HWS)

2. Morphologische Ursachen der HWS

3. Postoperative Dysphagie nach HWS-Operationen über einen anterioren Zugang

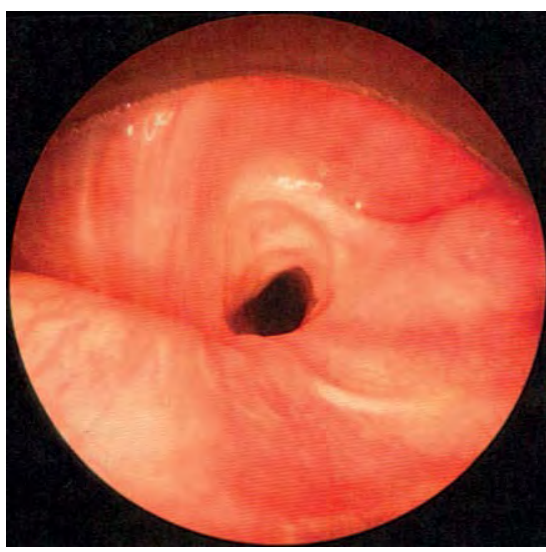

Abb. 13 Ringförmige Narbenstenose distaler Ösophagus.

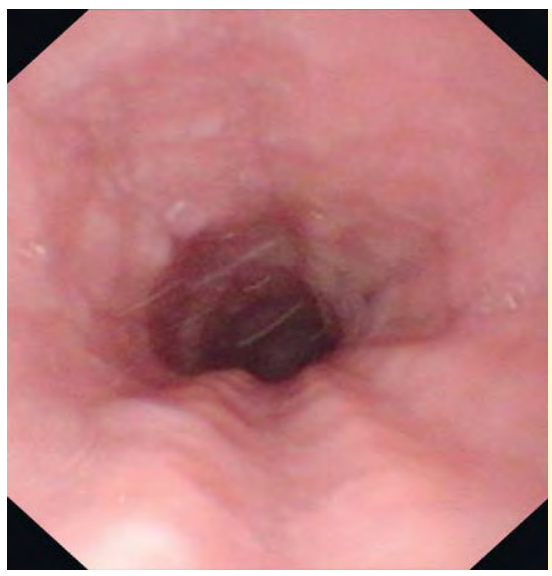

Abb. 14 Eosinophile Ösophagitis mit anhaltender Dysphagie. Z.n. mehrfachen Ösophagoskopien mit Entfernung von Boli. Zunehmende Rigidität der Ösophaguswand mit Problemen des Bolustransportes in der ösophagealen Phase.

\subsubsection{Funktionelle Störungen der Halswirbelsäule mit} Dysphagie

Zervikogene Schluckstörungen und ein Globusgefühl (Fremdkörpergefühl im Rachen bzw. Hals, unabhängig von der Nahrungsaufnahme) werden häufig durch eine funktionelle, reflektorische Störung der Halswirbelsäule (FSH) hervorgerufen. FSH sind fast ausschließlich in der oberen HWS und den Kopfgelenken lokalisiert. Sie entwickeln sich entweder unabhängig von pathomorphologischen Störungen oder als deren Folge und sind normalerweise vollständig reversibel. Bei Persistenz können sie aber auch in pathomorphologische Veränderungen übergehen. In der Regel sind C2/3 und C3/4 die verantwortlichen Kennsegmente für die funktionellen Schluckstörungen (FSS). Ursachen der FSH sind falsche Bewegungsmuster, degenerative Verände- 
rungen oder Traumata, Schleuderverletzungen und Distorsionen. Auch Schädelverletzungen können zu schweren und persistenten FSH und FSS führen $[44,45]$.

FSS können mit Globus-, Kloß- oder Fremdkörpergefühl einhergehen. Die Patienten beschreiben das Gefühl, über eine Schwelle schlucken zu müssen. Dies kann auch mit einem Enge- oder Beklemmungsgefühl im Hals kombiniert sein. Es besteht keine objektivierbare Ursache für die Schluckhemmung. Psychische Faktoren können für die Entwicklung und Unterhaltung der FSS mit verantwortlich sein. Seifert beschreibt jedoch die vorschnelle Einstufung als psychogener „Globus nervosus sive hystericus“ als fehlerhaft und therapeutisch ineffizient [46]. Außerdem muss differenzialdiagnostisch zunächst eine organische Ursache und hier vor allem eine Tumorerkrankung ausgeschlossen werden.

Die Verspannungen der am Zungenbein ansetzenden Muskeln mit Druckdolenz im Sinne einer Hyoidtendopathie sind durch Palpation nachweisbar. Die FSH wird durch die manualtherapeutische Untersuchung der HWS-Segmente diagnostiziert.

\subsubsection{Morphologische Ursachen der Halswirbelsäule mit Dysphagie}

Morphologische Veränderungen der HWS können entzündliche, degenerative und traumatische Ursachen haben und betreffen Patienten jenseits des 50. Lebensjahres.

Hierbei unterscheidet man nach Schröter-Morasch Veränderungen, die die Pharynxhinterwand in Form und Funktion beeinträchtigen sowie zu einer Einengung des Spinalkanals führen. Letztere kann zur Kompression der Medulla und/oder peripherer Nerven und der entsprechenden neurologischen Symptomatik mit Beeinträchtigung des Schluckaktes führen [47].

Degenerative Erkrankungen des Skelettsystems, wie bspw. Spondylitis ankylosans, Arthrosis deformans oder diffuse idiopathische skelettale Hyperostose sowie die hereditäre multiple Exostosenbildung können retropharyngeale Raumforderungen durch Hyperostosenbildung ausbilden. Asymptomatische zervikale Osteophyten sind jedoch auch in 20 - 30 \% der Bevölkerung nachweisbar [48].
Die Flexibilität des Pharynxschlauches erlaubt, dass Deformitäten zwar sichtbar aber nicht symptomatisch sind. Die Schluckstörung entwickelt sich erst bei Koordinationsstörungen mit Einschränkungen des Bolustransportes durch eine verminderte Motilität. Nach Strasser et al. [49] wird eine Dysphagie mit Aspiration bei Patienten beobachtet, deren zervikale Osteophyten größer als $1 \mathrm{~cm}$ sind.

Folgende Punkte wirken sich auf den Schluckablauf aus [47]:

1. Mechanische Blockierung der Boluspassage, besonders für feste Speisen.

2. Behinderung der Dorsalwärtsneigung der Epiglottis und damit Behinderung des vollständigen Verschlusses des Larynxeinganges.

3. Beeinträchtigung der pharyngealen Muskelkontraktion. Ein Fremdkörpergefühl beim Schlucken wird oftmals als Symptom der Dysphagie beklagt. Die Dysphagie kann in diesen Fällen auch mit Kopf- oder Gesichtsschmerzen verknüpft sein. Knöchern-knorpelige Vorwölbungen der zervikalen Wirbelkörper oder auch ligamentäre Ossifikationen, wie bei der diffusen idiopathischen Skeletthyperostose (M. Forestier) ( $\bullet$ Abb. 15a, b) in den Pharynx und/oder zervikalen Ösophagus, stellen mechanische Hindernisse dar, die den Schluckakt behindern können. Die prominenten Osteophyten im Bereich der Wirbelkörper C2-4 fallen regelhaft im Rahmen der starren oder flexiblen Endoskopie auf. Durch den insuffizienten Abschluss des Larynxeinganges durch prominente Osteophyten der Wirbel C3/4, C4/5 kann eine intradeglutitive Aspiration von Flüssigkeiten bedingt werden. In der Computertomografie oder dem Röntgen der Halswirbelsäule kann die Diagnose erhärtet werden. Differenzialdiagnostisch ist stets eine Tumorerkrankung auszuschließen.

Weiterhin können Fibrosierungen oder auch Spasmen eine Dysphagie durch den Boluskontakt im Bereich der Osteophyten begünstigen.

Seidler et al. geben 4 Gründe für die Dysphagie bei einer Hyperostosis an [50].

1. Unvollständiger Schutz der oberen Atemwege durch eingeschränkte Beweglichkeit der Epiglottis, große Osteo-
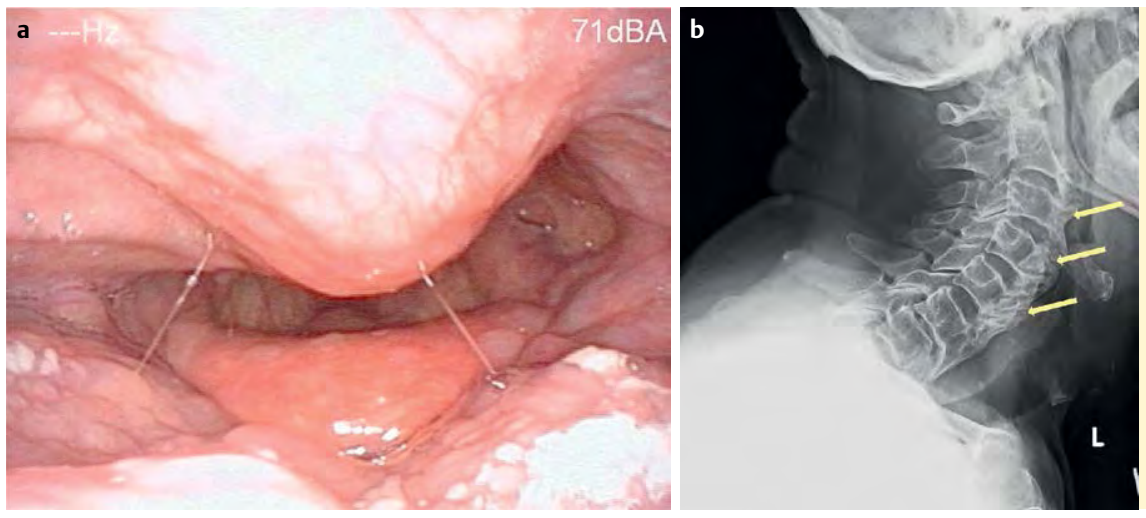

Abb. 15 Spondylitis hyperostotica (M. Forestier) eines 75-jährigen Patienten, Dysphagie durch prominente Hyperostosen der HWS. Endoskopiebild zeigt eine deutliche mittige Vorwölbung der Rachenhinterwand mit enger Beziehung zum Zungengrund und Epiglottis a. Seitliche Röntgenaufnahme der HWS des gleichen Patienten mit sichtbaren knöchernen Anbauten an den Wirbelkörpern und ligamentären Strukturen (C2-6, siehe Pfeile) $\mathbf{b}$.
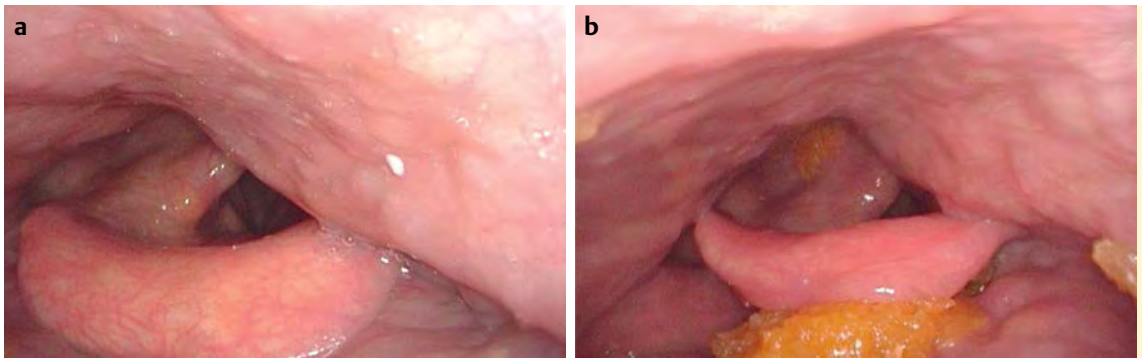

Abb. 16 53-jähriger Patient mit HWS-Versteifung und Revisionsoperation. Persistente Dysphagie. a Linke Pharynxseitenwand wölbt sich über den Larynx mit Kontakt zur Epiglottis. b Kleiner Bolus in der Vallecula (physiologisch). Transportstörung des Karottenbolus im rechten Hypopharynx. 
phyten können die Abwärtsbewegung der Epiglottis blockieren

2. Unvollständiger Glottisschluss beim Schlucken bedingt durch Osteophyten, die die Adduktion der Aryknorpel und damit der Stimmlippen behindern

3. Einschränkung der Elevation und anterioren Bewegung des Kehlkopfes

4. Mechanische Behinderung des Transports des Nahrungsbolus durch Vorwölbung der Hypopharynxhinterwand.

\subsubsection{Postoperative Dysphagie bei HWS-Operationen}

Operationen der Halswirbelsäule (HWS) bei Traumata, Dekompressionen mit Diskektomien oder Wirbelfusionen können zu einer Beeinträchtigung der pharyngealen Phase des Schluckaktes führen. Dies tritt vor allem nach HWS-Operationen über den anterioren Zugang auf. Bei diesem operativen Standardzugang gibt Fountas die postoperative Dysphagie als häufigste Komplikation mit 9,5\% bei 1015 Patienten an [51]. Nach Mukherjee [52] ist die Ätiologie am ehesten multifaktoriell. Die Dysphagie wird durch pharyngoösophageale Denervation, zervikale Weichteilschwellung, Vernarbung und Druckschäden [53] hervorgerufen ( $\bullet$ Abb. 16a, b).

In der ersten postoperativen Woche treten PD nach anterioren HWS-Operationen in bis zu $79 \%$, nach einem Monat bis zu 50 $56 \%$ und nach einem Jahr bis zu 13-21 \% auf [54, 55]. Risikofaktoren sind hohes Alter, weibliches Geschlecht und die Multilevelchirurgie $[55,56]$. Bartholome und Schröter-Morasch empfehlen für diese Patienten eine sorgfältige postoperative Nachbetreuung, damit aufgrund klinischer, endoskopischer und röntgenologischer Untersuchungen frühzeitig die weitere funktionelle oder chirurgische Therapie eingeleitet werden kann [47].

\subsection{Dysphagie durch Hypersalivation und Xerostomie} Eine Hypersalivation wird durch folgende Ursachen ausgelöst:

- Zentral-neurologische Störungen (amyotrophe

Lateralsklerose, M. Parkinson, Apoplex, infantile

Zerebralparese)

- Periphere Störungen der Nn. V und VII

- Orofaciale Dysfunktion

- Gastro-ösophagealer Reflux

- Tumorchirurgie/iatrogene Speichelfisteln

- Medikamentennebenwirkung (Neuroleptika, Haloperidol)

- Mundwasser, Zahnpasta

- Idiopathisch.

Eine Xerostomie kann folgende Ursachen haben:

- Zentrale Ursachen: M. Parkinson, Depression, psych. Faktoren, SHT, ZNS-Tumoren

- Medikamente: trizyklische Antidepressiva, Sedativa und Tranqillanzien, Antihistaminika, Antihypertensiva, Chemotherapeutika

- Diabetes mellitus, Exsikkose

- Bestrahlung (> 25 Gy irreversible Schädigung)

- Primäres/sekundäres Sjögren-Syndrom

- Sarkoidose, Amyloidose

- Kinder: zystische Fibrose

- Resektion Speicheldrüsen.

\subsection{Neurologische Krankheitsbilder}

Für Patienten mit Dysphagie bei neurologischer Grunderkrankung ist immer eine interdisziplinäre Zusammenarbeit erforderlich, um die konservative oder operative Therapie anhand der
Symptomatik und Prognose festzulegen. Folgende Gruppen wurden identifiziert [57]:

- Neurovaskuläre Erkrankungen (z. B. ischämischer Schlaganfall)

- Neurodegenerative Erkrankungen (z.B. M. Parkinson)

- Neuromuskuläre Erkrankungen (z. B. amyotrophe Lateralsklerose, Polymyositis)

- Neurotraumatologische Erkrankungen (z. B. Schädel-HirnTrauma)

- Neuroonkologische Erkrankungen (z. B. Gliome, paraneoplastische Erkrankungen)

- Neuroinfektiologische Erkrankungen (z. B. Hirnstammenzephalitis)

- Altersbedingte Veränderungen der Schluckfunktion (Presbyphagie).

\subsection{Medikamentenbedingte Dysphagien}

Ganz allgemein haben annähernd alle Medikamente durch ihre pharmakologischen Wirkprofile die Potenz, eine Dysphagie auszulösen oder zu verstärken. Faktoren wie reduzierter Allgemeinzustand, erhöhtes Alter, multiple Medikation, anatomische Besonderheiten des Magen-Darmtraktes, degenerative Hirnveränderungen und/oder psychiatrische Auffälligkeiten sind Risikofaktoren [1]. Auch Überdosierungen bei nicht beachteter reduzierter Leber- und Nierenleistung mit Kumulationseffekten können medikamentenassoziierte Schluckstörungen initiieren oder verstärken. Darüber hinaus können Arzneistoffe untereinander kumulative Effekte haben [58].

Medikamente können eine direkte Wirkung auf die Schluckfunktion verursachen, wenn sie eine Wirkung u. a. auf die Strukturen haben, die unmittelbar am Schluckvorgang beteiligt sind, wie z. B. auf die Muskulatur des Ösophagus. Sie haben eine indirekte Wirkung, wenn sie die Voraussetzungen für den Schluckvorgang beeinflussen, wie z. B. eine medikamentös verursachte Xerostomie [59].

Medikamentenassoziierte Einflüsse auf die Schluckfunktion werden häufig nicht ausreichend wahrgenommen, stillschweigend akzeptiert oder nicht erkannt [60]. Medikamentöse Wirkungen auf den Schluckakt sind besonders kritisch, wenn anatomisch-funktionelle Veränderungen, wie z. B. eine chronische Ösophagitis oder Ösophagusstriktur [61], vorliegen, verschiedene Medikamente gleichzeitig gegeben werden und/oder bereits Schluckprobleme über einen längeren Zeitraum bekannt sind.

\subsubsection{Lokale Wirkung auf die Schluckfunktion \\ (oral medication-induced esophageal injury)}

Arzneistoffe können durch den direkten Kontakt mit der Ösophagusmukosa während des Schluckvorgangs zu lokalen Entzündungen und Ulzerationen führen und werden als eigenständiger Symptomkomplex unter dem Begriff „oral medicationinduced esophageal injury“ OMIEI oder „drug-induced esophageal injury“ (DIEI) zusammengefasst. Die Leitsymptome sind eine Dysphagie, es können ein Fremdkörpergefühl, ein Globusgefühl und eine Odynophagie bestehen, in vielen Fällen wird über eine ösophageale Transportstörung berichtet. Instinktiv nehmen die Betroffenen bei soliden Konsistenzen deshalb vermehrt Flüssigkeit auf. Die OMIEI ist am ehesten bei älteren Patienten, bei Patienten mit reduziertem Allgemeinzustand und Patienten mit Motilitätsstörungen oder anatomischen Veränderungen des Ösophagus zu erwarten [59]. Eine Übersicht der Medikamente, die vorrangig eine OMIEI auslösen können, zeigt $\odot$ Tab. 4. 
Tab. 4 Medikamente, die eine OMIEl auslösen können (modifiziert nach $[1,62])$.

- Antibiotika vorrangig Tetrazykline (z. B. Doxycyclin), Trimethoprim (z. B. Bactrim), Makrolide (z. B. Clindamycin)

- Nichtsteroidale Antirheumatika (NSAR)

- Azetylsalizylsäure (ASS)

- Bisphosphonate wie Alendronate (Fosamax)

- Sildenafil (Phosphodiesterase-5-Hemmer, Vasodilatator, z. B. bei pulmonaler Hypertension, arteriellem Hypertonus)

- Zidovudine (Retrovir)

- Kalium

- Theophyllin

- Chinidin (Antiarrhythmika)

- Eisensulfate (in Eisenpräparaten)

- Vitamin C

Nicht nur der Wirkstoff, sondern auch die Art und Größe der Tabletten und die verwendete Flüssigkeitsmenge haben einen Einfluss auf eine OMIEI [63]. Außerdem kann eine Latenzzeit zwischen dem erstmals eingenommenen Medikament und dem Auftreten einer OMIEI bestehen [64].

\subsubsection{Systemische Medikamentenwirkung auf die Schluckfunktion (Klassifikation)}

3.6.2.1 Zentral-sedierend/bewusstseinsverändernd wirksame Medikamente. Als wichtige Substanzgruppe sind Wirkstoffe zu nennen, die die zentrale Erregbarkeit und Vigilanz bewusst reduzieren. Dazu zählen alle Antikonvulsiva, aber auch viele Antidepressiva. Darüber hinaus können Antiallergika sowie Analgetika, insbesondere mit opiatähnlicher Wirkung durch ihre sedierende Komponente mit Auswirkungen auf Reflexsteuerung, Sensorik und muskuläre Koordination die Schluckfunktionen negativ beeinflussen. Bei Benzodiazepinen wird eine Auswirkung auf die laryngeale Schluckaktivität vermutet [65]. In der Behandlung von kindlichen Epilepsien wurden bei Nitrazepam muskuläre Koordinationsstörungen der krikopharyngealen Region mit Aspiration als Todesfolge bei Kindern beschrieben [66]. $\bigcirc$ Tab. 5 fasst die wichtigsten Medikamente zusammen, die durch eine zentrale Wirkung Dysphagien auslösen oder verstärken können.

3.6.2.2 Zentral wirksame Medikamente mit peripherer Nebenwirkung.

Xerostomie. Die Xerostomie ist die vorrangige periphere Nebenwirkung zentral wirkender Medikamente. Dazu gehören die trizyklischen Antidepressiva (z. B. Amitriptylin), SerotoninWiederaufnahmehemmer [1] und opiathaltige Schmerzmittel [67]. Da sich im Alterungsprozess ohnehin die Speichelkonsistenz verändert und die Speichelproduktion reduziert, kann eine medikamentös induzierte Xerostomie beträchtliche Auswirkungen insbesondere auf den Bolustransport haben [68]. 0 Tab. 6 zeigt eine Zusammenstellung von Medikamenten, die typischerweise eine Xerostomie auslösen können.

Die bei ACE-Hemmern häufig zu findende, typische Nebenwirkung von chronischem Husten kann indirekt eine Auswirkung auf das Schluckprofil haben oder aber eine Aspiration vortäuschen. ACE-Hemmer sind jedoch die einzigen Medikamente, die nachweislich die Schluckfunktion verbessern können. Arai et al. beschrieben ein reduziertes Risiko für eine Aspirationspneumonie bei Stroke-Patienten, sodass sogar bei arterieller Normotonie eine ACE-Behandlung empfohlen wurde [69].
Tab. 5 Zentral wirksame Medikamente, die eine Dysphagie auslösen oder verstärken können (nach $[1,18]$ ).

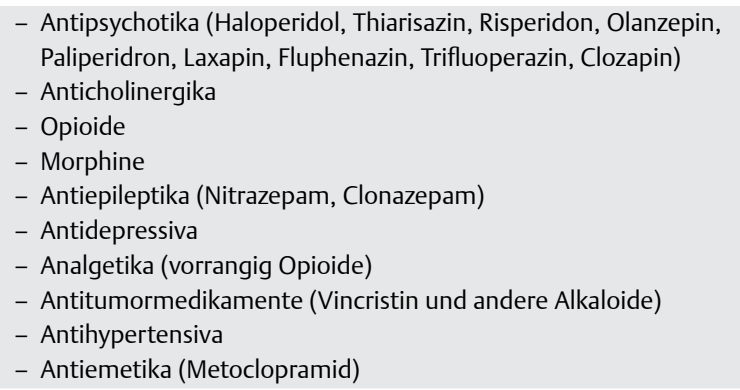

Tab. 6 Medikamente, die eine Xerostomie auslösen können (modifiziert nach $[18,62])$.

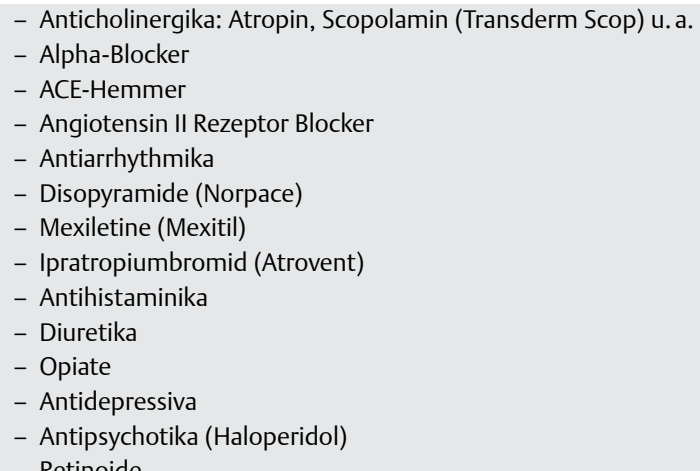

Tab. 7 Medikamente und Stoffe, die eine Ösophagusmotilitätsstörung oder Tonusreduktion des unteren Ösophagussphinkters (Myopathie mit Dysphagie) auslösen können (modifiziert nach [62]).

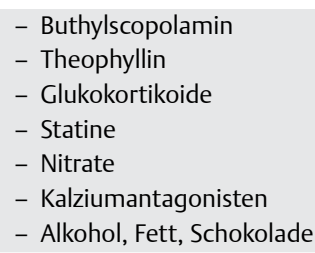

Neuro-muskuläre Wirkung. Neuroleptika reduzieren Koordination und Muskelaktivität von Pharynx und Ösophagus, und können Dyskinesien mit unkoordiniertem Schluckablauf hervorrufen [70]. Einzelne Fälle von Aspirationen mit tödlichem Ausgang wurden beschrieben [1].

Dopaminantagonisten, die z.B. beim Parkinson-Syndrom eingesetzt werden, können spät einsetzende Dyskinesien hervorrufen oder aber selbst ein Parkinsonssyndrom auslösen und eine bereits bestehende Schluckstörung auch negativ beeinflussen [68], obwohl sie die muskuläre Koordination verbessern sollen. Medikamente können eine Muskelschwäche und/oder eine Myositis auslösen ( $\bullet$ Tab. 7). Dazu gehören insbesondere Lipidsenker (Statine) sowie Kolchizin [71]. Die am längsten bekannte und am häufigsten auftretende medikamenteninduzierte Myopathie wird durch Kortikosteroide ausgelöst (Steroidmyopathie) [72]. Zu den Medikamenten oder Wirkstoffen, die durch ihre Darreichungsform als Injektion oder Applikation lokal wirken, gehören Botulinumtoxine, deren Injektion bei spasmodischer Dysphonie oder zervikaler Dystonie Schluckschwierigkeiten verursachen können [73]. Weiterhin reduzieren Oberflächenanästhetika (in manchen Lutschpastillen enthalten) Halsschmerzen und 
Tab. 8 Synopsis der grobmotorischen und orofazialen motorischen Entwicklung.

\begin{tabular}{|ll}
\hline $\begin{array}{l}\text { Alter (Monate) } \\
0-1\end{array}$ & $\begin{array}{l}\text { Meilensteine der Grobmotorik } \\
\text { Beugetonus, Greifreflex, Suchen } \\
\text { zunehmende Kopfkontrolle } \\
\text { Kopfkontrolle in Bauchlage }\end{array}$ \\
\hline 3 & Armkontrolle, Drehen beginnt \\
\hline 4 & Extension/Flexion, Drehen, Füße zum Mund \\
\hline 5 & $\begin{array}{l}\text { Abstützbewegung der Arme, gezieltes Greifen und } \\
\text { Wechsel zwischen beiden Händen }\end{array}$ \\
\hline $7-9$ & Robben, Krabbeln, stabiles Sitzen \\
\hline $10-12$ & Pinzettengriff, Horizontale zur Vertikalen \\
\hline $13-18$ & Laufen, präzises Greifen \\
\hline $19-24$ & $\begin{array}{l}\text { Treppensteigen mit nachgestelltem Bein } \\
\text { Verfeinern, Hüpfen, Umgang mit Schere }\end{array}$ \\
\hline $24-36$ &
\end{tabular}

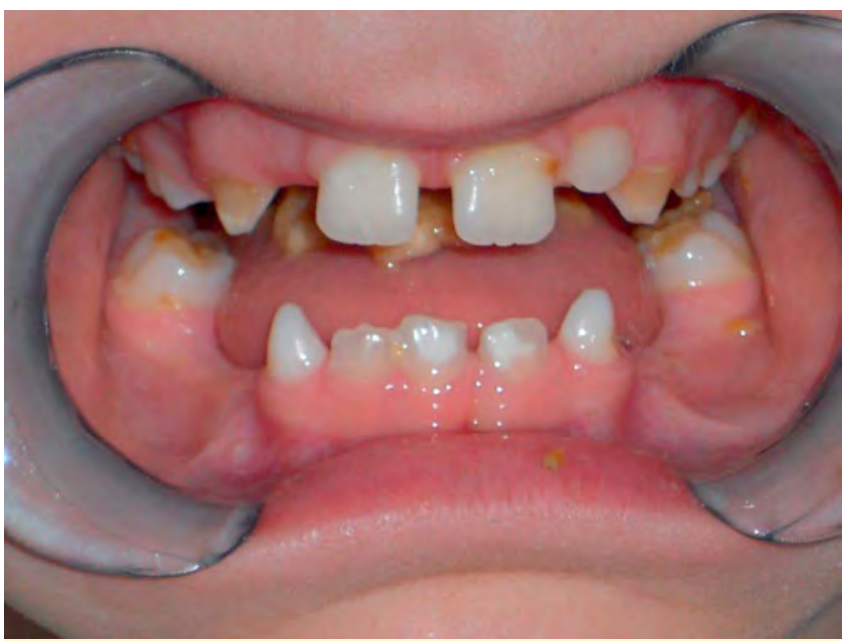

Abb. 17 Dysphagie mit Störung der oralen Phase. Anteile des Bolus werden beim Schlucken durch die Zungenprotrusion in das Vestibulum oris nach vorne und zur Seite transportiert. Zahnfehlstellung, Schmelzdefekte.

Schmerzen bei Aphtose. Sie werden ebenfalls vor Endoskopien der oberen Luft- und Speisewege zur reversiblen Minderung der Oberflächensensibilität eingesetzt.

\subsection{Dysphagie im Kindesalter}

Eine altersgerechte Entwicklung sensorischer und motorischer Funktionen ist die Voraussetzung für eine normale Schluckfähigkeit. Die $\bullet$ Tab. 8 zeigt eine Synopsis der grobmotorischen und der orofazialen Entwicklung [74].

\subsubsection{Ursachen von Dysphagien bei Kindern}

Kindliche Dysphagien können neurologische, strukturelle, funktionelle oder verhaltensbedingte Ursachen haben [75, 76]

( $\odot$ Abb. 17, 18). Dazu gehören traumatische Hirnschäden und -blutungen, neuromuskuläre Ursachen (infantile Zerebralparese, spinale Muskelatrophie Typ II) sowie Folgen von Frühgeburtlichkeit (Koordinationsstörung von Schluck- und Atemfunktion mit sensomotorischen Defiziten, bronchopulmonale Dysplasie, Apnoe-Bradykardie-Syndrom, Stimmlippenlähmung nach Herzoperationen, Laryngomalazie, nekrotisierende Enterokolitis nach Antibiotikagabe) [2]. Ein gastro-ösophagealer Reflux [77] sowie akute und chronische, häufig immunvermittelte Mukositiden (z.B. eosinophile Ösophagitis, M. Crohn) erschweren den Bolustransport [78-80] und sind mit Husten, Pneumonien, Ap-

\section{Meilensteine der orofazialen Motorik}

Erstes Saugmuster (Sucking): Saug-Schluck-Sequenz 1x/s spontane Mundöffnung, Lippenschluss zunehmend zunehmende Flexion des Nackens: pharyngealer Weg wird weiter, Laute werden gebildet

Lippen- u. Zungenkontrolle, erstes Füttern möglich

Brei, Flüssigkeiten aus Becher, Hand wird mit Nahrung zum Mund geführt Zweites Saugmuster (Sucking), Zahnen, Würgereflex abnehmend, Lippenrundung

koordinierte Zungen-, Lippen-, Kieferbewegungen, laterale Kaubewegung, alleine trinken

eigenständiges Essen mit Fingern, Abbeißen, diagonal-rotatorisches Kauen koordiniertes Phonieren, alle Konsistenzen

Löffel eigenständig, Trinken mit Lippenschluss, rotierendes Kauen Löffelführung mit Fingern, Becher einhändig

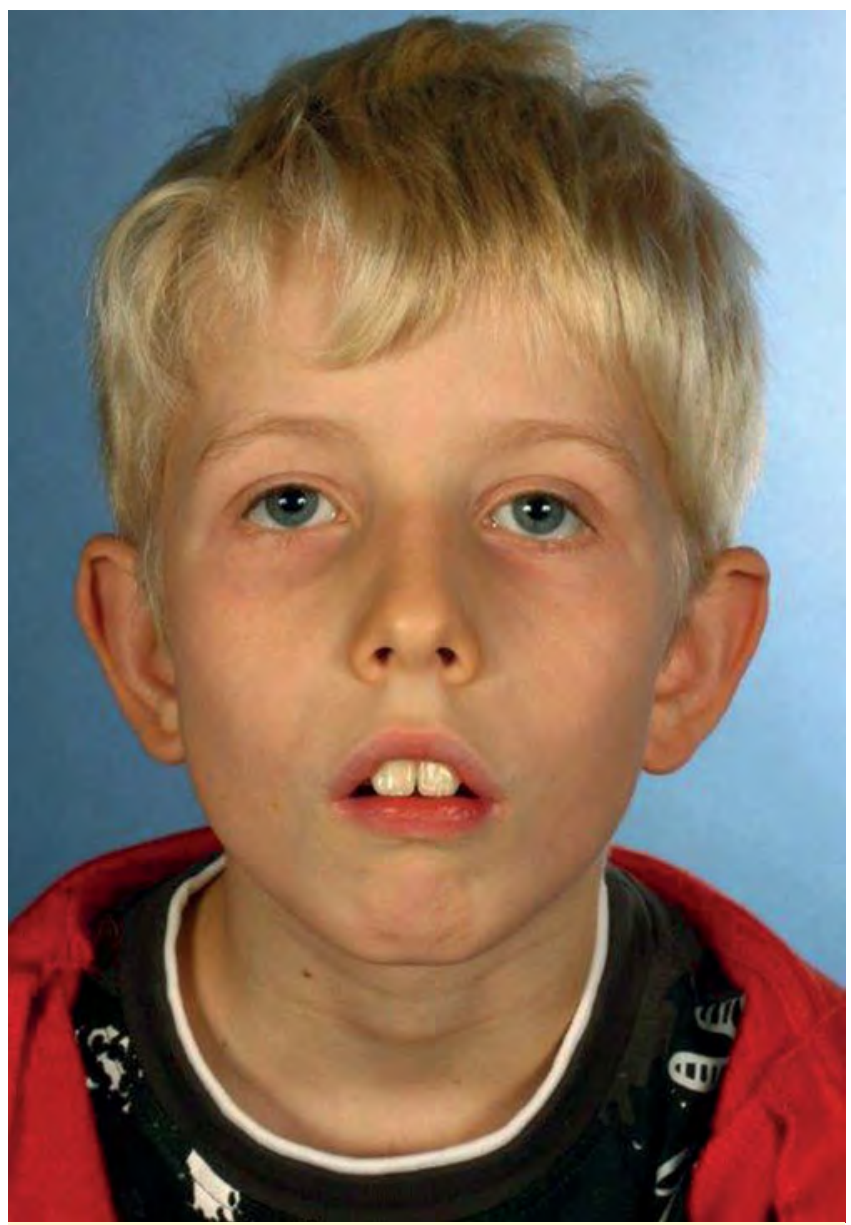

Abb. 18 Facies adenoidea mit orofazialer Dysfunktion (myofunktionelles Syndrom) mit reduziertem orofazialen Tonus, dadurch mangelndem Mundschluss, kompensatorischer Anspannung des Musculus mentalis („Nadelkissen“), müder Gesichtsausdruck.

noen, Ess- und Gedeihstörungen verbunden. Auch eine OMIEI kann bereits bei Kindern vorliegen, insbesondere wenn chronische Krankheiten langfristig oral medikamentös behandelt werden (z. B. juvenile rheumatoide Arthritis) oder eine Chemotherapie bei Malignomen erfolgt. Häufig kann eine OMIEI und die damit verbundenen unspezifischen Verhaltensveränderungen mit wählerischem/ungenügendem Essverhalten als charakterliche Eigenart fehlinterpretiert werden. Gedeihstörungen und 
Tab. 9 Verschiedene Ursachen kindlicher Schluckstörungen (modifiziert nach Kühn [81] und Biber [90]).

Anatomische Ursachen
- Lippen-/Kiefer-/
Gaumenspalten
- Zungenfehlbildungen
- Mikro-/Retrognathie
- Choanalatresie
- Laryngomalazie
- tracheoösophageale Fistel
- Stenosen/Atresie des
Ösophagus/Larynx

Genetische Ursachen

Syndrome:

- Trisomie 21

- Trisomie 18

- Möbius-Sequenz

- Apert Syndrom

- Prader-Willi-Syndrom

- Pierre-Robin-Sequenz

- Beckwith-Wiedermann-Syndrom

- Cri-du-chat-Syndrom

- CHARGE Syndrom

- Treacher-Collins-Syndrom

- 22q11-Mikrodeletion

- Cornelia-de-Lange-Syndrom

- Meningomyelozele mit

Arnold-Chiari II- Malformation

$\begin{array}{ll}\text { Neurologische Ursachen } & \text { Andere Ursachen } \\ \text { - Hirn-/Rückenmarksfehlbildungen } & \text { - Medikamente } \\ \text { - intrauterine Intoxikationen } & \text { - eosinophile Ösophagitis } \\ \text { Perinatal: } & \text { - langfristige Immunschwäche } \\ \text { - hypoxisch-ischämische Hirnschädigung } & \text { - langfristige Sondenernährung } \\ \text { - Frühgeburtlichkeit } & \text { - Obstruktion der obere Atemwege } \\ \text { Postnatal: } & \text { - Tracheotomie } \\ \text { - Tumore } & \text { - Langzeitbeatmung/Intubation } \\ \text { - Infektionen (z. B. Meningitis, Enzephalitis, } & \text { - intrauterine Wachstumsretardierung } \\ \text { Polymyelitis) } & \text { - intrauterine Infektion } \\ \text { - Schädel-Hirn-Trauma } & \text { - Gedeihstörung } \\ \text { - akute Bilirubinanämie } & \text { - gastropharyngealer Reflux } \\ \text { - degenerative Hirnerkrankungen } & \\ \text { - metabolische Enzephalopathien } & \\ \text { - Erkrankung des zentralen Nervensystems } & \\ \text { (z. B. Zerebralparesen) } & \\ \text { - Myasthenia gravis } & \end{array}$

rezidivierendes Erbrechen sind unter Umständen die einzigen Anzeichen [80].

Weiterhin können kraniofaziale Fehlbildungen (fetales Alkoholsyndrom, CHARGE-Syndrom - Akronym für coloboma, heart anomalies, atresia of the choanes, retarded growth, genital hypoplasia, ear anomalies -, Pierre-Robin-Sequenz, Spaltbildungen), anatomische Besonderheiten der gesamten Schluckstraße (z.B. Velumspalten, Ösophagusengen) und die Folgen von Tracheotomien ursächlich für eine Schluckstörung im Kindes- und Jugendalter sein. Frühgeborene, auch ohne zerebrale Komplikationen, haben ein erhöhtes Risiko für Dysphagien über das Kleinkindalter hinaus [81]. Allgemein zeigen deutlich entwicklungsretardierte Kinder nicht selten lebenslang Kau- und Schluckschwierigkeiten, die eine kalorisch-ausreichende orale Ernährung nicht gewährleisten.

Von den Dysphagien abzugrenzen sind Ess-, Fütter- und Interaktionsstörungen [82], wobei gerade bei Säuglingen und Kindern diese idiosynkratische Sichtweise der Komplexität der Störung nicht gerecht wird und eine Schluckstörung sogar ein Symptom einer Fütterstörung sein kann [83]. Die Ansicht, „Fütterstörungen“ und „Dysphagie“ als getrennte Entitäten zu sehen, wird aktuell revidiert zugunsten von „Schluck- und Fütterstörungen bei entwicklungs-beeinträchtigten Kindern“ („swallowing and feeding disorders in developmental disabilities“, SFD-DD), worunter sowohl dysphagische Symptome mit Problemen in den einzelnen Schluckphasen als auch Fütterprobleme mit Problemen beim Essen subsummiert werden.

Die Dysphagieproblematik ergibt sich aus der Persistenz primitiver Reflexe, die die Entwicklung physiologischer Schluckmuster hemmen. Die ineffektive orofaziale Koordination und eingeschränkte Pharynxmotilität sowie die ungenügende Schluckreflextriggerung führen zu einer quantitativ und qualitativ ungenügenden Schluckaktivität [84]. Typisches Symptom einer prä-, peri- oder postnatalen Hirnschädigung ist die infantile Zerebralparese. Sie ist insbesondere bei Frühgeborenen sowie aufgrund von Geburtskomplikationen anzutreffen und weist ein sehr variables Erscheinungsbild auf [85].

Die Inzidenz von Schluckstörungen bei Kindern mit Zerebralparesen wird mit bis zu $99 \%$ angegeben [86 - 88]. Die Prävalenz von Schluckstörungen in der Patientengruppe mit Di- und Hemiparesen wird mit 25 - 30\% angegeben, während bei tetraparetischer und extrapyramidaler Bewegungsstörung bis zu $80 \%$ der Betroffenen Störungen in der oralen Nahrungsaufnahme ha- ben $[83,85]$. Bei Kindern mit Syndromen (z. B. Trisomie 21) sind Schluckstörungen in mehr als 50 \% vorhanden [89].

- Tab. 9 zeigt eine Übersicht über die Ursachen kindlicher Schluckstörungen.

Die Auswirkungen sind vielfältig: eingeschränkte Lebensqualität, Abhängigkeit von Sondenernährung und Trachealkanülen, Aspiration und Aspirationspneumonien [91], Dehydratation sowie Fehl- und Unterernährung stellen schwere Folgen dar, die besonders am reifenden Gehirn auf lange Sicht zu Entwicklungsstörungen führen und vital bedrohlich verlaufen können [92, 93]. Daher hat die frühe Diagnosestellung und Ableitung von Therapiekonzepten im Säuglings- und Kindesalter einen hohen Stellenwert.

\subsubsection{Symptomatik/Klinik von Dysphagien bei Kindern} Offensichtliche Zeichen einer Dysphagie bei Kindern sind Erbrechen oder Regurgitation, Husten während oder nach der Nahrungsaufnahme und erhöhte Passagezeiten während der oralen Schluckphase. Oft ergeben sich sekundäre Hinweise auf eine Schluckstörung, wie mangelndes Interesse am Essen, Muskelanspannungen während der Nahrungsaufnahme, verlängerte Fütterzeiten, Ausspucken von Nahrung und Flüssigkeiten, gurgelnde Atemgeräusche oder eine belegte Stimme, Atemprobleme und Gedeihstörungen [74]. Bei einer stillen Aspiration fehlen erkennbare Symptome [94]. Chronische bronchopulmonale Erkrankungen können Anzeichen für eine unerkannte kindliche Schluckstörung mit Aspiration sein. Bei einer milden Symptomatik werden diese Kinder in der Praxis häufig lange mit bronchodilatativen oder kortikoidhaltigen Medikamenten (fehl-)behandelt. Bei Kindern mit Erkrankungen des neuromuskulären Übergangs, verschiedenen anderen Grunderkrankungen und als Nebenwirkung von Medikamenten kann im Verlauf der Nahrungsaufnahme eine zunehmende Ermüdung die Gefahr einer Dysphagie erhöhen.

\subsubsection{Fütterbeobachtung, Screening und klinische Schluckuntersuchung bei Kindern}

Klinische Assessments stellen Methoden dar, die auch bei Kindern als alleinige diagnostische Methode keine ausreichend hohe Validität bezüglich der Vorhersage einer Aspiration zeigen und als unzureichend angesehen werden [95]. International existieren nur wenige, englischsprachige, standardisierte und validierte Verfahren, und nur das „Dysphagia Disorders Survey 
(DDS)“ und das „Schedule for Oral Motor Assessment (SOMA)“ werden mit einer ausreichenden klinischen Aussagekraft eingeschätzt [96]. Deutschsprachig liegen keine vergleichbaren validierten Instrumente vor. Die Übertragung von Screeningverfahren für Erwachsene auf Kinder ist aufgrund des Ablaufs und der zu schluckenden Mengen nicht zulässig.

Falls es die Untersuchungssituation und der Allgemeinzustand des Kindes zulassen, sollte eine Fütterbeobachtung erfolgen. Bei Kindern, die anamnestisch offensichtlich aspirieren, muss darauf verzichtet werden.

Vergleichbar zum Ablauf bei Erwachsenen erfolgt auch bei Kindern eine Einschätzung der Vigilanz, der Atmung, des Körpertonus und der Körperhaltung, insbesondere der Kopf- und Rumpfkontrolle. Dies muss bezogen auf die Meilensteine der motorischen, kognitiven und orofazialen Entwicklung (u.a. Saugmuster, Saugpolster, Lippenschluss, Kiefer- und Zungenkontrolle, Kaubewegungen, Hand-Mundkoordination, $\bigcirc$ Tab. 8) beurteilt werden.

\subsubsection{Standardisiertes Vorgehen bei Anamnese und Untersuchung}

3.7.4.1 Anamnese bei Kindern. Folgende Fragen sollten mit den Bezugspersonen geklärt werden (modifiziert nach [97]):

- Wie lange dauern Mahlzeiten (mehr als $30 \mathrm{~min}$ ?)?

- Empfinden Sie oder das Kind die Mahlzeiten als stressbehaftet?

- Zeigt das Kind Atemprobleme während der Mahlzeiten?

- Wie ist die Gewichtsentwicklung des Kindes?

- Hat Ihr Kind eine (Aspirations-)Pneumonie erlitten?

In der Anamnese werden auch Fragen zu Schwangerschaft, Geburt, allgemeiner Entwicklung (motorisch, schluckmotorisch, sprachlich), Allergien, Medikamente, Vorerkrankungen, Vorbehandlungen und Therapien gestellt. Ein besonderer Schwerpunkt liegt auf der aktuellen Ernährungs- und Esssituation. Es sollte auch nach oralen Gewohnheiten wie Schnullerverwendung, Zähneknirschen und besonderen oralen Vorlieben gefragt werden.

3.7.4.2 Vorbereitung für die flexibel-endoskopische Schluckuntersuchung (FESU) bei Kindern. Anlage einer Pulsoxymetrie. Die Schluckfrequenz sollte beobachtet werden (normal ist ein Schluck pro Minute, während der Endoskopie 3-mal pro Minute [98]). Der Spiegelbefund im Kopf-Hals-Bereich und der Zahnstatus werden erhoben, wobei der Schwerpunkt auf der nasalen Anatomie, den peri- und enoralen sowie oropharyngealen motorischen und sensorischen Funktionen liegt. Nach Abschwellen der nasalen Schleimhaut mit altersgerecht dosierten Nasentropfen erfolgt eine Schleimhautanästhesie der vorderen Nasenabschnitte mit Gel (z. B. Xylocain-Gel, 0,1 ml pro Seite). Durch diese Maßnahme kann die Kooperativität der Kinder erheblich gesteigert und eine Traumatisierung für nachfolgende Untersuchungen verhindert werden. Die Schleimhautanästhesie der vorderen Nasenabschnitte mit einem Gel beeinflusst die Schluckfähigkeit nicht [99]. Auf anästhesierendes Spray sollte verzichtet werden, da es schmerzhaft ist und die Ausdehnung des anästhesierten Areals durch die Verneblung nicht kontrolliert werden kann. Bei kardio-pulmonal sehr instabilen Kindern muss vor Gabe von abschwellenden Nasentropfen und anästhesierendem Gel zunächst mit den behandelnden Pädiatern eine Risikoabschätzung erfolgen. Eine Sedierung (z. B. mit Chloralhydrat) sollte nicht erfolgen. Obwohl Schlucken eine basale Fähigkeit ist, ist bei der untersuchten Patientengruppe davon auszu- gehen, dass auch eine geringe Sedierung das Untersuchungsergebnis negativ beeinflusst.

Neben der Bezugsperson hat sich die Anwesenheit einer weiteren Person, z. B. des behandelnden Schlucktherapeuten, als sehr positiv erwiesen und wird von der American Speech-LanguageHearing Association (ASHA) empfohlen [100]. Im interdisziplinären Team kann so ein individuelles Behandlungskonzept unmittelbar erarbeitet werden. Da Eltern die Schluckfunktion ihrer Kinder häufig besser einschätzen als sie tatsächlich ist $[87,101,102]$, ist es ratsam, zusätzliche Informationen von Therapeuten wie Kinder- und Jungendärzten, Logopäden, aber auch von Erziehern oder Lehrern zu erhalten.

3 Nahrungstexturen werden hergestellt und zunächst ein Nativschluck pro Konsistenz durchgeführt. Für Krümeliges hat sich homogenes Brot ohne Rinde bewährt. Angedicktes wird mit Wasser und Andickungsmittel hergestellt, und als Flüssigkeit wird stilles Wasser verwendet. Aber auch auf spezielle Vorlieben der Kinder kann eingegangen werden. Falls nötig, kann dann auf handelsübliche Lebensmittelfarben zurückgegriffen werden. Dies hat den Vorteil, dass auch bei retrospektiver Evaluation der gespeicherten Daten die Farbe für die geprüfte Konsistenz steht. Einen besonders guten Kontrast zur Schleimhaut bieten die Farben Grün und Blau. Die Boli sollten kräftig angefärbt werden, sodass sie im Becher dunkel erscheinen, um auch kleine Residuen erkennen zu können. Das ist besonders wichtig, um die laryngeale Penetration und die eventuelle Benetzung der Stimmlippen nicht zu übersehen.

Von Methylenblau zum Anfärben des Bolus zur besseren Visualisierung wurde in den USA berichtet, dass sie bei septischen Patienten zu allergischen Reaktionen geführt habe [98]. Es wurde vorgeschlagen, auf Farbe zu verzichten und bei nicht ausreichender Sichtbarkeit den Bolus mit Barium zu mischen. Barium haftet stark an der Schleimhaut und am Endoskop und verhindert so die Beurteilung weiterer Boli. Sollte Barium aspiriert werden, kann es zu Pneumonien führen, sodass Barium hier nicht zum Einsatz kommen sollte. Von der Verwendung von Götterspeise wird dann abgeraten, wenn Flüssigkeiten aspiriert werden können, da sie sich bei längerer Verweildauer im Mund verflüssigt und nicht die intendierte Konsistenz geprüft wird. Handelsübliche Andickungsmittel sollten bevorzugt werden (z.B. Nestargel, ThickenUp, Thick\&Easy, Nutilis).

3.7.4.3 Standardisierte Durchführung der FESU bei Kindern. Für die instrumentelle Untersuchung der kindlichen Dysphagie [74] stehen, wie bei Erwachsenen, im Wesentlichen die Videofluoroskopie und die FESU zur Verfügung. Beide Verfahren werden oft ergänzend eingesetzt $[103,104]$. Bei der videofluoroskopischen Schluckuntersuchung (VFES) stellt die Strahlenbelastung des Kindes einen deutlichen Nachteil dar, insbesondere, wenn wiederholte Untersuchungen erfolgen müssen. Diese sind aber in der Regel immer erforderlich, wenn evidenzbasiert gearbeitet wird und der Erfolg einer Therapie untersucht werden soll. Daten zur Strahlenexposition und zur effektiven Dosis wurden von Weir et al. vorgelegt [105]. Dabei wurde die Strahlendosis für ältere Kinder als akzeptabel, für jüngere Kinder als zu hoch eingeschätzt. Bei motorisch und kognitiv beeinträchtigten Kindern kann die FESU in der Regel besser eingesetzt werden. Unter Berücksichtigung von Studien mit Kindern, die auf eine annähernd 100 \%ige Übereinstimmung von VFES und FESU hindeuten [106], spricht insbesondere bei Kindern viel dafür, zunächst regelmäßig eine FESU und erst bei damit nicht zu klärenden Befunden/ Fragen eine VFES durchzuführen. Als Komplikationen werden 
eine Hypersalivation, eine Epistaxis sowie eine Synkope durch einen vaso-vagalen Reflex oder ein Laryngospasmus beschrieben. Diese sind jedoch selten, und die Methode wird auch im ambulanten Bereich als sicher eingeschätzt $[107,108]$. Die Ausstattung mit einem Notfallkoffer für Kinder sowie regelmäßige Kinder-Reanimationsschulungen sind dennoch immer ratsam.

3.7.4.4 Ablauf. In Anlehnung an die Untersuchung bei Erwachsenen [103] erfolgt die Ruhebeobachtung (Speichel, Morphologie, Spontanbewegungen usw.), die Funktionsprüfungen ohne Nahrung (Phonation, Speichelschluck, Reinigungsfunktionen usw.), die Funktionsprüfungen mit Nahrung (verschiedene Bolusvolumina und -konsistenzen) sowie die Überprüfung von Schlucktechniken und Reinigungsmanövern. Empfohlen wird, den Rumpf und die Beine des Kindes, je nach Alter, in ein großes Laken einzuwickeln und das Kind auf den Schoß zu platzieren, wobei die Arme am Körper anliegen, sodass sich die Beine des Kindes zwischen den Beinen der Bezugsperson befinden [74]. Dies vermittelt ein sicheres Gefühl und gewährleistet eine stabile Position während der Untersuchung. Gleichzeitig wird unkontrollierten Armbewegungen vorgebeugt. Eine zusätzlich anwesende Person kann bei Bedarf den Kopf von hinten unterstützen.

Es empfiehlt sich ein Endoskop zu benutzen, mit dem einerseits eine gute Sicht und andererseits eine minimale Irritation der Schleimhaut gewährleistet ist, damit Verletzungen vermieden werden und die Kinder kooperativ bleiben. Bei Früh- und Neugeborenen eignen sich dazu flexible Endoskope mit einem Durchmesser von ca. $2 \mathrm{~mm}$. Neben der Beurteilung der anatomisch-morphologischen und funktionellen Befunde des Naso-Oro-Hypopharynx und des Larynx (Stimmlippenbeweglichkeit) liegt ein besonderes Augenmerk auf Speichelresiduen oder einer Speichelaspiration sowie auf spontane Reinigungsmechanismen (Räuspern, Husten). Liegt eine stille Aspiration vor, sollte die Untersuchung beendet oder weiteres Schlucken nur mit größter Vorsicht und kleinsten Mengen Angedicktem weitergeführt werden. Bei Speichelresiduen kann versucht werden, durch Aufforderung zum Husten oder durch transorales oder transnasales Absaugen eine bessere Übersicht zu erlangen. Die verschiedenen Konsistenzen „,angedickt“, „flüssig“ und „krümelig“ werden meist mit einem dem Alter des Kindes angepassten Löffel geprüft, beginnend mit kleinen Mengen und gegebenenfalls steigernd. Die Beurteilung erfolgt nach der Penetrations-Aspirations-Skala nach Rosenbek [109]. Die Untersuchung wird videodokumentiert. Durch leichtes Touchieren der laryngealen Epiglottisfläche spitzennah und Beobachtung der Reaktion mit Husten oder Würgen bzw. der Anwendung definierter Luftdruckstöße [110] kann eine Aussage zur laryngealen Sensibilität erfolgen.

Falls das Kind permanent schreit, ist wegen der Aspirationsgefahr und der Wahrscheinlichkeit der falsch-positiven Einschätzung keine orale Gabe der Prüfsubstanzen möglich. Sollten orale Residuen vorhanden sein, werden diese nach der Untersuchung entfernt.

Grundsätzlich ist man als Untersucher geneigt, Trachealkanülen und Magensonden vor einer FESU zu entfernen, um eine möglichst ungestörte Larynxelevation zu ermöglichen, die mögliche Kompression des Ösophagus durch den Cuff zu reduzieren und die vermeintliche laryngeale und hypopharyngeale Schleimhautirritation durch eine Magensonde zu vermindern. Studien bei Erwachsenen mit einer Schluckstörung haben gezeigt, dass die Schluckfähigkeit bei endoskopischer Evaluation mit und ohne Trachealkanüle gleich ist $[75,94]$. Tracheotomierte Kinder
Tab. 10 Anamnesebogen bei Schluckstörungen.

Patientenbefragung (ggf. Therapeut, Angehörige, Pflegepersonal)

subjektive Einschätzung

Haben Sie Schluckbeschwerden? Seit wann? allgemeine Hinweise auf eine Schluckstörung Haben Sie seit der Erkrankung an Gewicht abgenommen? Wieviel?

Leiden oder litten Sie an einer Lungenentzündung? Wie oft?

Haben Sie häufiger Infekte?

Haben Sie unklare Temperaturerhöhungen?

Husten Sie mehr als früher?

Müssen Sie oft räuspern?

Bemerken Sie eine stärkere Verschleimung? Hat sich Ihre Stimme verändert? (z. B. heiser, gurgelnd)? Haben Sie ein Kloß/Fremdkörpergefühl im Hals?

Haben Sie Angst vor dem Schlucken?

Haben Sie beim Schlucken Schmerzen?

Brauchen Sie länger zum Essen als früher?

Nehmen Sie jetzt beim Essen/Trinken eine andere Kopfhaltung ein?

Hinweise auf eine gestörte orale Phase

Läuft Flüssigkeit aus dem Mund?

Können Sie schlechter kauen?

Haben Sie ein taubes Gefühl im Mundbereich?

Ist Ihr Mundraum trocken?

Empfinden Sie kalte Speisen weniger als früher?

Empfinden Sie warme Speisen weniger als früher?

Haben Sie Probleme die Nahrung in den Rachen zu bringen?

Müssen Sie vor dem Hinunterschlucken husten?

Bleibt nach dem Schlucken

Nahrung $\square$ Flüssigkeit $\square$ im Mund zurück?

Hinweise auf eine gestörte pharyngeale Phase

Haben Sie das Gefühl, dass ihr Rachen trocken ist?

Kommt beim Schlucken etwas in die Nase?

Haben Sie das Gefühl, dass nach dem Schlucken

Nahrung $\square$ Flüssigkeit $\square$ im Hals steckt?

Müssen Sie beim Hinunterschlucken husten?

Müssen Sie nach dem Hinunterschlucken husten?

Müssen Sie die Speisen wieder hochräuspern und ausspucken?

Klingt Ihre Stimme nach dem Schlucken anders?

Hinweise auf eine gestörte ösophageale Phase

Haben Sie das Gefühl, dass die Nahrung in der

Speiseröhre stecken bleibt?

Haben Sie Schmerzen hinter dem Brustbein?

Kommt Ihnen Nahrung wieder hoch?

sauer $\square$ nicht sauer $\square$

Verspüren Sie ein Brennen hinter dem

Brustbein $\square$ im Hals $\square$ (Sodbrennen)?

Müssen Sie nach der Mahlzeit husten?

Konsistenzabhängige Symptome

Haben Sie Schwierigkeiten beim Schlucken von:

Speichel?

Flüssigkeiten?

pürierte Speisen?

festen Speisen?

Welche Speisen/Getränke bereiten Ihnen besondere

Probleme?

Nein Ja 
können sowohl mit als auch ohne Kanüle, Kinder mit einer nasogastralen Sonde mit und ohne Sonde untersucht werden [111]. Sollte es sich bei den untersuchten Kindern als am geringsten problematisch erweisen, die Trachealkanüle zu entfernen, sollte die Untersuchung mit Kanüle durchgeführt werden. Magensonden werden in aller Regel belassen, da von einer Veränderung des Ergebnisses nicht auszugehen ist. Ergibt sich während der Untersuchung, dass die Larynxelevation anscheinend wesentlich behindert ist oder dass es postdeglutitiv zu einem Rückfluss des Bolus kommt, muss zunächst bei vorhandenem Cuff mit entblockter und dann, bei bleibendem postdeglutitivem Passagehindernis, mit entfernter Kanüle untersucht werden.

\section{Diagnostik der Dysphagie}$$
\nabla
$$

\subsection{Anamnese bei Dysphagie}

Die Anamnese gibt Hinweise auf mögliche Ursachen der Dysphagie, ihre klinischen Symptome und deren Folgen ( $\bullet$ Tab. 10). Sie kann entweder durch Befragung von Patienten, Angehörigen, Therapeuten und Pflegepersonal oder durch Einsicht in die Krankenakte erhoben werden. Wichtig sind folgende Informationen:

- Grunderkrankung, Erkrankungszeitpunkt

- bisherige Behandlung

- bei Tumorpatienten: Art und Ausmaß des Primärtumors und der chirurgischen, radiologischen und chemotherapeutischen Intervention

- bei neurologischen Patienten: Läsionsort und -ausmaß, bisheriger Verlauf, Medikation

- Ernährungsmodus im Verlauf: parenteral, enteral über Sonden, oral mit Modifikation der Nahrungsaufnahme, der Beschaffenheit der Nahrung

- Zeichen von Mangelernährung und Exsikkose

- Respiratorischer Status im Verlauf: Beatmung, Tracheotomie, pulmonale Komplikationen

- Hinweise auf Refluxsymptomatik

- Medikamenteneinnahme (eine Vielzahl von Medikamenten kann eine Dysphagie verstärken oder verursachen, z. B. durch Mundtrockenheit, extrapyramidale Bewegungsstörungen, gastrointestinale Störungen, Myopathien [71,112], siehe Kapitel 3.6 Medikamentenbedingte Dysphagien)

Tab. 11 Unterscheidungsmerkmale peripherer und zentraler Bewegungsstörungen der Schluck-/Sprechorgane.

\begin{tabular}{|c|c|c|}
\hline $\begin{array}{l}\text { Beurteilungskri- } \\
\text { terium }\end{array}$ & Periphere Parese & Zentrale Parese \\
\hline $\begin{array}{l}\text { Willkürbewe- } \\
\text { gungen }\end{array}$ & aufgehoben & $\begin{array}{l}\text { aufgehoben oder } \\
\text { beeinträchtigt }\end{array}$ \\
\hline $\begin{array}{l}\text { reflektorische und } \\
\text { emotionale Bewe- } \\
\text { gungen }\end{array}$ & aufgehoben & erhalten \\
\hline Muskeltonus & erniedrigt & $\begin{array}{l}\text { Anfangs erniedrigt, } \\
\text { später erhöht }\end{array}$ \\
\hline Muskelatrophie & $\begin{array}{l}\text { bei längerem Bestehen } \\
\text { vorhanden, an der Zunge } \\
\text { möglicherweise Ausbil- } \\
\text { dung eines Sulkus }\end{array}$ & nicht vorhanden \\
\hline Faszikulationen & möglicherweise vorhanden & nicht vorhanden \\
\hline Hyperkinesen & nicht vorhanden & $\begin{array}{l}\text { möglicherweise } \\
\text { vorhanden }\end{array}$ \\
\hline
\end{tabular}

- Beschwerden bei der Atmung, beim Schlucken von Speichel und Sekret, beim Essen und Trinken.

Die Symptome einer Dysphagie der oralen, pharyngealen oder ösophagealen Phase können mit einem Fragebogen erfasst werden [17]. Nach Wright und Ellis [113] korrelieren die subjektiven Beschwerden der Patienten und die Lokalisation einer oropharyngealen Dysphagie stark.

\subsection{Allgemeine Beurteilung, klinische Untersuchung}

Der Schluckvorgang ist ein hochkomplexer Bewegungsablauf, dessen Störungen in der Regel multifaktoriell bedingt sind. Für eine pathophysiologisch orientierte Therapie müssen die Ursachen durch eine erweiterte ärztliche Untersuchung des Kopf-Hals-Bereiches mit der Überprüfung schluckrelevanter neurologischer Einzelfunktionen gefunden werden. Dazu gehört eine orientierende Prüfung von Hirnleistung, Kommunikationsfähigkeit und Gesamtmotorik (Haltung, Kopf- und Rumpfkontrolle, Paresen, Ataxie, Hyperkinesen, Dystonien; Beurteilung von HWS, Hals- und Nackenmuskulatur).

Die Diagnose, ob es sich um eine periphere Nervenläsion oder um eine supranukleäre zentrale Schädigung handelt, beruht hauptsächlich auf der genauen Beurteilung der reflektorischen und willkürlichen Beweglichkeit sowie des Muskeltonus ( $\bullet$ Tab. 11). Beide Formen können an Lippen, Zunge, Gaumensegel und Kehlkopf einseitig oder beidseitig auftreten [114,115].

Nicht immer lässt sich die Symptomatik eindeutig abgrenzen, da z. B. bei einer Hirnstammschädigung sowohl das zweite, periphere, als auch das erste, zentrale Motoneuron betroffen sind. In Phasen der Rückbildung können wechselnde klinische Bilder vorhanden sein.

\subsubsection{Untersuchung von Lippen, Kiefer, Zunge und vorderer Mundhöhle}

4.2.1.1 Strukturen. Die Strukturen werden nach der Form und Symmetrie der Lippen und der Zunge, dem Kiefer- und Zahnstatus und der Schleimhautbeschaffenheit beurteilt. Patienten nach Tumorbehandlung haben z. T. massive Strukturdefizite und Zahndefekte. Ausgedehnte Vernarbungen und Ödeme können Kopfhaltung, Mundbodenbeweglichkeit und Larynxelevation erheblich behindern.

Nach intensivmedizinischer Behandlung, während der keine Zahnprothese getragen wird, kommt es bereits nach wenigen Wochen zu Veränderungen des Alveolarkamms.

4.2.1.2 Beweglichkeit. Die Untersuchung der Beweglichkeit erfolgt zunächst durch die Aufforderung zur Ausführung willkürlicher Bewegungen:

- Lippenschluss, -spitzen, -spreizen

- Kiefer öffnen, schließen, seitlich verschieben, rotieren

- Zunge herausstrecken, anheben, rückführen, kreisen, an den Lippen entlangführen, Zungenspitze in die Wangentaschen drücken.

Nach einer Studie von Leder an über 3900 Patienten ist eine reduzierte Zungenbeweglichkeit ein Risikofaktor für Aspirationen, unabhängig von der Grunderkrankung [116].

Der Muskeltonus beeinträchtigt die Beweglichkeit wenn er zu hoch oder zu niedrig ist. Der Unterkiefer kann sowohl durch einen niedrigen Tonus der Kaumuskulatur als auch - häufiges Symptom nach Schädel-Hirn-Trauma - durch einen zu hohen Tonus der den Unterkiefer absenkenden und retrahierenden Muskulatur herabhängen. Der Lippenschluss kann ungenügend sein wegen einer zu hohen (Lippenretraktion) oder zu geringen 
Tonisierung, wobei der Bewegungsradius nicht notwendigerweise eingeschränkt sein muss.

4.2.1.3 Überprüfung der oralen Sensibilität. Bei verminderter Sensibilität besteht immer die Gefahr einer eingeschränkten Boluskontrolle. Nicht selten klagen Patienten mit taktilen Sensibilitätsstörungen darüber, sich auf Zunge und Wangeninnenseite zu beißen und dies dann als sehr schmerzhaft zu empfinden. Die taktile Sensibilität wird durch Berührung mit einem Watteträger geprüft.

Die Temperaturwahrnehmung in der Mundhöhle wird nur orientierend durch die Berührung mit einem kleinen Larynxspiegel geprüft, der entweder in kaltes oder in warmes Wasser getaucht wurde. Schwellenbestimmungen sind methodisch sehr aufwändig, und ihre klinische Relevanz ist fraglich.

\subsubsection{Untersuchung von hinterer Mundhöhle, Velum und} Pharynx

4.2.2.1 Mundhöhle. In der hinteren Mundhöhle ist vor allem bei Patienten nach Tumortherapie der Zunge, des Gaumens, des Pharynx und Larynx auf Schwellungen, Substanzdefekte und Narben zu achten. Vorwölbungen der Rachenhinterwand müssen im Hinblick auf Veränderungen der HWS, gelegentlich aber auch auf Entzündungen und Abszesse abgeklärt werden.

4.2.2.2 Velum. $\mathrm{Zu}$ hoch tonisierte Gaumenbogenmuskeln geben dem Gaumenbogen eine scharfkantige Kontur und schaffen einen großen Abstand des Gaumensegels von der Rachenhinterwand. Eine ungenügende velare Tonisierung ist am ventralen Vorschieben des Gaumensegels während einer forcierten Ausatmung („Flattern im Luftstrom“) erkennbar.

Eine zentrale Parese liegt vor, wenn bei der sekundenlang angehaltenen willkürlichen Phonation von [a:] und bei mehrmaliger Phonation von [a] keine Anhebung des Velums, aber eine reflektorische Anhebung beim Versuch, den Palatal- oder den Würgreflex auszulösen, bzw. beim Lachen, Weinen oder Gähnen beobachtet werden kann.

Lässt sich kein Würgreflex auslösen (häufiges Symptom z.B. nach Schädel-Hirn-Trauma), kann man mit dem flexiblen Endoskop untersuchen, ob beim Schluckvorgang eine Velumanhebung und ein nasopharyngealer Abschluss erfolgt. Wenn dies nicht der Fall ist, kann von einer peripheren Parese ausgegangen werden.

4.2.2.3 Pharynx. Die Kontraktion der Pharynxmuskulatur wird gleichzeitig mitgeprüft, sowohl bei der willkürlichen Phonation als auch bei Auslösung eines Würgreflexes. Bei dieser Untersuchung lässt sich jedoch nicht eindeutig einschätzen, ob die Kontraktion normal oder eingeschränkt ist. Dies kann nur anhand des videoendoskopischen, radiologischen bzw. manometrischen Befundes beurteilt werden.

4.2.2.4 Sensibilität und Reflexauslösbarkeit im Oropharynx. Störungen der Sensibilität findet man, wie bereits beschrieben:

- Nach Tumorchirurgie - Läsionen peripherer Nerven im Narbengebiet des Pharynx und Larynx bzw. im Bereich von Lappentransplantaten

- Nach Bestrahlungen

- Nach Schlaganfall - Zungengrund, Gaumenbögen, Velum und Rachenhinterwand sind meist einseitig betroffen.

Neuere Untersuchungen ergaben, dass bei einseitigen Großhirninfarkten Sensibilitätsstörungen der Gaumenbögen mit verlän- gerter Latenz der Schluckreflextriggerung und Aspirationen einhergehen können $[117,118]$. Eine wichtige Rolle des Schluckkortex scheint es also zu sein, eine intakte Sensibilität der Mundhöhle zu garantieren und die orale mit der pharyngealen Phase zeitlich so zu koordinieren, dass kein vorzeitiger Übertritt von geschlucktem Material bzw. keine Aspirationen stattfinden [103]. Die Auslösbarkeit des Würgreflexes ist individuell sehr unterschiedlich. Fehlt er, scheint ein höheres Risiko für das Vorliegen einer Dysphagie zu bestehen [119], doch darf bei vorhandenem Würgreflex keinesfalls immer auf einen ungestörten Schluckablauf geschlossen werden.

Es gibt auch Menschen, die keinen Würgreflex haben. Bereits beim Berühren der Lippen mit einem Strohhalm setzt in Erwartung von Flüssigkeit oder Speisen eine Hypästhesie des Pharynx und Larynx ein. Dies ermöglicht, dass während des Schluckens z. B. die laryngeale Epiglottisfläche berührt werden kann.

\subsection{Fragebogen-Assessments, Screeningverfahren und klinische Dysphagiediagnostik}

In der klinischen Routine ist die apparative Ausstattung für Schluckuntersuchungen nicht immer vor Ort verfügbar, sodass oft zunächst strukturierte Fragebögen und Screeningverfahren zum Einsatz kommen, um eine Entscheidung zum weiteren diagnostischen und therapeutischen Ablauf zu treffen. Klinische Schluckuntersuchungsverfahren gehen in der Regel einer apparativen Diagnostik voraus und werden meist von Logopäden/ Sprachtherapeuten durchgeführt. Für alle genannten Verfahren existieren bisher keine Richtlinien.

\subsubsection{Fragebogen-Assessments}

Die vorhandenen Assessments gehen entweder auf die Bereiche „Symptome einer Dysphagie“ oder „Lebensqualität“ ein. Erstere eignen sich eher, um Dysphagie-spezifische Symptome zu erfragen, letztere dazu, die Gesamtsituation der Patienten mit ihren Dysphagie-bedingten Einschränkungen zu erfassen. Damit kommen sie der Betrachtungsweise von Erkrankungen nach der „International Classification of Functioning, Disability and Health“ der Weltgesundheitsorganisation entgegen.

Zu den symptomspezifischen Assessments gehören das 10-Item Assessment Tool (EAT-10) [120,121], das Sydney Swallowing Questionnaire (SSQ) $[122,123]$ sowie der Munich Dysphagia Test - Parkinson's Disease (MDT-PD) [124].

Für die Erhebung von Lebensqualität-spezifischen Bereichen und ihren Einschränkungen werden das MD Anderson Dysphagia Inventory (MDADI) [125], der Swal-QOL sowie Swal-Care [126-128], der Dysphagia Handicap Index (DHI) [129], die Performance Status Scale for Head and Neck Cancer Patients (PSSHN) $[130,131]$, sowie das Functional Assessment of Cancer Therapy-Head and Neck Scale (FACT-H\&N) [130] angewandt.

\subsubsection{Screeningverfahren}

Screeningverfahren dienen dazu, potentiell gefährdete Patienten zu identifizieren und ohne großen Zeitaufwand das Aspirationsrisiko einzuschätzen [132], um sie einer weiterführenden Diagnostik zuzuführen. Sie sollten schnell, einfach, sicher, mit einer hohen Aussagekraft und für den Patienten wenig belastend im Sinne einer bedside-Untersuchung auch von medizinischem Personal, das hierfür speziell eingearbeitet wurde, durchgeführt werden können. Für erste, schnelle Entscheidungen sollen sie eine Schluckstörung mit einer Sensitivität (sicherer Nachweis) und Spezifität (sicherer Ausschluss) von jeweils $70 \%$ erfassen [133]. Ein Goldstandard der gängigen Screeningverfahren exis- 
tiert bisher nicht. Dennoch haben Screeningverfahren einen hohen Stellenwert, da die Verfügbarkeit einer apparativen Schluckdiagnostik (FESU und VFES) nicht überall und kurzfristig gegeben ist, aber dennoch umgehend eine möglichst zuverlässige Einschätzung zur Ernährung eines Patienten getroffen werden muss. Über 50 \% der Patienten, die aspirieren, husten nicht. Daher bleibt in der klinischen Diagnostik ein hoher Anteil an „stiller Aspiration“ unentdeckt und ist ein limitierender Faktor für die Sensitivität.

Kontraindikationen für Aspirationsschnelltest sind:

- bereits bekannte Aspirationszeichen

- pathologische Lungenbefunde

- schwere Bewusstseinsstörungen.

Für das Screening neurogener Dysphagien stellt der Timed test [137] ein validiertes Instrument dar. Dabei sollen $150 \mathrm{ml}$ kaltes stilles Wasser so schnell wie möglich aus einem Glas getrunken werden. Die Zeit, die Schluckfrequenz, Restmengen im Glas sowie Husten und die Stimmqualität nach dem Trinken werden registriert. Angegeben werden die $\mathrm{ml} / \mathrm{s}$ sowie das durchschnittliche Volumen pro Schluck (ml). Eine Schluckgeschwindigkeit $<10 \mathrm{ml} / \mathrm{s}$ wird als pathologisch angesehen. Für das Item „Schluckgeschwindigkeit“ wird eine Sensitivität von $96 \%$ und eine Spezifität von $69 \%$ angegeben. Die Autoren weisen darauf hin, dass der Test nicht für Patienten mit einer gravierenden Schluckstörung und offensichtlicher Aspiration geeignet ist.

In der akuten Schlaganfallphase stehen das durch eingearbeitetes Pflegepersonal durchführbare Standardized Swallowing Assessment (SSA) $[138,139]$ bzw. das Gugging Dysphagia Bedside Screening [140] oder der durch Sprachtherapeuten/Logopäden durchzuführende „Daniels-Test" [13] zur Verfügung. Letzterer soll mit einer Sensitivität von 92 \% und einer Spezifität von 67 \% anhand der 6 klinischen Symptome: Dysphonie, Dysarthrie, beeinträchtigtes willkürliches Husten, abgeschwächter oder fehlender Würgereflex, Husten und Änderung der Stimmqualität nach dem Wasserschluck, eine Aspiration vorhersagen. Sind 2 von 6 Symptomen vorhanden, wird eine apparative Schluckdiagnostik empfohlen. Als weiterer Test kommt der 3-Ounce Water Swallow Test von Suiter und Leder [141] zum Einsatz. Positiv ist der Test, wenn Husten, ein Erstickungsanfall oder eine feuchte Stimme auftreten oder der Test abgebrochen werden muss. Der Test sollte wegen der großen Wassermenge erst nach vorheriger Austestung mit einer geringeren Menge durchgeführt werden.

Aspirationsschnelltests sind wegen des geringen Zeitaufwands und geringer Kosten als Entscheidungshilfe für Sofortmaßnahmen geeignet. Bei anamnestischem oder klinischem Verdacht auf eine Aspiration/Dysphagie ist die erste Untersuchung die FESU. Damit kann die Frage nach weiteren Untersuchungsschritten, ausführliche klinische oder radiologische Diagnostik, und deren Reihenfolge beantwortet werden.

Für die Dysphagie nach Schlaganfall existiert eine große Anzahl unterschiedlicher Tests. So werden der 50-ml-Wasser-Test [47], der 3-Ounce Water Test (=90-ml-Wasser-Schluck-Test) [142], der Kidd Water Test [143], der Burke Dysphagia Screening Test [144], der Wassertest nach Daniels [13], das Bedside swallowing Assessment (BSA) [145], das Standardized Swallowing Assessment(SSA) [138, 139], das Massey Bedside Swallowing Screening [146], der Nishiwaki-Score [147], das Gugging Swallowing Screening [140], der Volume-Viscosity Swallow Test (V-VST) [148], das Toronto Bedside Swallowing Screening (TOR-BSST) [149], die Modified Mann Assessment of Swallowing Ability (MMASA) [150], der Acute Stroke Dysphagia Screen [151] sowie der Cough Test [152] durchgeführt.
Eine relativ hohe Sensitivät/Spezifität und eine relativ einfach Durchführbarkeit weisen der 90-ml-Wasser-Test [141,142] und der Gugging Swallowing Screen (GUSS, Prüfung verschiedener Konsistenzen) [140] auf.

4.3.2.1 90- ml-Wasser-Test (3-Ounce Water Swallow Test). Der Patient soll $90 \mathrm{ml}$ Wasser ohne Unterbrechung aus einem Glas mit oder ohne Strohhalm trinken. Aspirationshinweise und Abbruchkriterien sind:

- Austrinken der gesamten Flüssigkeitsmenge nicht möglich

- Auftreten von Husten oder Erstickungsanfall bis zu 1 min nach Testende

- Gurgelnde, feucht-belegte Stimmqualität.

Da die Menge von $90 \mathrm{ml}$ pulmonal gefährdend sein kann, sind Vortests mit kleineren Wasserschlucken (1, 3, 5, $10 \mathrm{ml})$ sinnvoll. Zeigen sich keine Symptome, liegt mit hoher Wahrscheinlichkeit auch keine Aspiration dünnflüssiger Konsistenzen vor. Wegen der geringen Spezifität sind viele Patienten mit auffälligem Testergebnis dennoch nicht aspirationsgefährdet. Bei Aspirationshinweisen sollte in jedem Fall weiter diagnostiziert werden.

4.3.2.2 Gugging Swallowing Screen (GUSS). Die Voruntersuchung oder der indirekte Schluckversuch beinhalten die Überprüfung der Vigilanz (der Patient muss mindestens $15 \mathrm{~min}$ wach bleiben können), die Fähigkeit, Speichel zu schlucken, sich 2-mal willkürlich zu räuspern und/oder zu husten sowie die Erfassung von Drooling und Stimmänderungen während dieser Zeit. Im direkten Schluckversuch werden die Konsistenzen „breiig“/,flüsig“/,fest“ geprüft.

Als Hinweis auf eine Schluckstörung gilt dabei das Auftreten von mindestens einem der folgenden Symptome:

- Schlucken nicht möglich

- verzögerte Schluckinitiierung (Flüssigkeit $>2 \mathrm{~s}$, feste Nahrung $>10 \mathrm{~s}$ )

- Drooling

- unwillkürliches Husten vor, während oder nach dem Schlucken (bis 3 min später)

- Stimmänderungen nach dem Schlucken (Patient soll vor und nach dem Schlucken „0“ sprechen).

Nach einer 20-Punkte-Skala wird in 4 Schweregradstufen unterteilt.

Der Test beurteilt sowohl den Dysphagieschweregrad als auch das Aspirationsrisiko mit einer Sensitivität von $100 \%$. Bei einer Spezifität von 50 - 69\% ist eine hohe Rate falsch-positiver Ergebnisse zu erwarten. Folgerichtig wird bei Anzeichen einer leichtgradigen Dysphagie mit geringem Aspirationsrisiko die endoskopische Schluckdiagnostik empfohlen. Ein Protokollbogen mit Auswertung ist im Internet erhältlich [153].

Für die Schluckfähigkeit nach Tumorresektion von Kopf-HalsTumoren werden das Frankfurter Dysphagie-Screening (FraDySc) [154], der Water Swallow Screening Test [155] sowie das Mann Assessment of Swallowing Ability - Cancer (MASA-C) [156] beschrieben. Diese Screeningtests sind z. T. nicht validiert bzw. nicht ausreichend aussagekräftig. Es sollte deshalb bei Tumorpatienten mit Dysphagieverdacht die Schluckfunktion auf jeden Fall endoskopisch überprüft werden [157].

\subsubsection{Untersuchung von Patienten mit Trachealkanülen}

Bei Kanülenträgern kann aspiriertes Material relativ leicht abgesaugt und bei Anfärbung als solches identifiziert [134] bzw. durch den Glukose-Oxidase-Test [135] nachgewiesen werden. 
Ein positives Ergebnis weist in jedem Fall auf eine Aspiration hin, ein negatives Resultat lässt dagegen keine Schlussfolgerung zu, da diskrete Mengen aspirierten Materials zu häufig nicht erfasst werden.

Zum Anfärben eignet sich blaue Lebensmittelfarbe. Wegen möglicher Toxizität sollte Methylenblau nicht mehr verwendet werden $[98,136]$.

\subsection{Klinische Schluckuntersuchungsverfahren}

Unter dem Begriff klinische Schluckuntersuchung wird ein Untersuchungsablauf subsumiert, bei dem neben der schluckspezifischen Anamnese und der Beurteilung der Vigilanz und Compliance des Patienten alle für das Schlucken relevanten Strukturen inklusive der am Schlucken beteiligten Hirnnerven geprüft sowie Schluckversuche im Rahmen von Screeningverfahren durchgeführt werden [47, 158 - 160]. Bisher existiert kein einheitliches Vorgehen $[16,161]$, und es steht kein standardisiertes und ausreichend validiertes Verfahren zur Verfügung. Besonders gute Kenntnisse der Funktionsweisen von Trachealkanülen sind in diesem Zusammenhang von entscheidender Bedeutung. Als Beurteilung für die Ergebnisse aus klinischen Schluckuntersuchungsverfahren kommen u. a. der Nishiwaki-Score [147] und das Kölner Befundsystem für Schluckuntersuchungen [162] zum Einsatz.

\subsubsection{Stufenkonzepte}

Als strukturierte diagnostische Stufenkonzepte in deutscher Sprache sind das NOD-Stufenkonzept für Patienten mit neurogener oropharyngealer Dysphagie [163] sowie das Konzept nach Stix für geriatrische Patienten [164] beschrieben. Diese bedienen sich verschiedener Methoden für Anamnese, Screening, klinische Schluckuntersuchungsverfahren und apparative Schluckuntersuchungen. Ziel dieser Stufenkonzepte ist es, mit einer einheitlichen Terminologie und anhand festgelegter Kriterien Zuständigkeiten zu klären, Patienten mit Schluckstörungen mittels strukturierter Abläufe früh zu identifizieren und eine entsprechende Therapie einzuleiten.

\subsubsection{Endoskopische Untersuchungstechniken}

Für die Erfassung von Schluckstörungen hat sich die Videoendoskopie als effizientes Instrumentarium etabliert $[2,165-$ 168] und gilt heute als unverzichtbare Basisuntersuchung. Sie beeinträchtigt die Patienten nur gering.

Sie ist in der Erfassung von Residuen, Penetration und Aspiration gleichwertig effizient zur röntgenologischen Videofluoroskopie $[27,30,165,167,169$ - 178] und von großer klinischer Relevanz. So fiel die Anzahl der auftretenden Aspirationspneumonien in einem Zeitraum von 6 Monaten nach Einbeziehung der endoskopischen Schluckuntersuchung in das Dysphagiemanagement signifikant auf 0 [179].

Die Ziele der endoskopischen Untersuchung sind:

- Diagnostik und Abgrenzung struktureller und neurologischer Störungen und deren Charakteristik

- Entscheidung ob auf natürlichem Wege ausreichend Nahrung/Flüssigkeit in angemessener Zeit aufgenommen werden kann oder Sonden- oder parenterale Ernährung erwogen werden müssen

- Entscheidung ob Schutzmaßnahmen für die tiefen Atemwege erforderlich sind (Intubation, Tracheotomie)

- Vorliegen einer Indikation zur Speichelreduktion

- Entscheidungshilfen für Schluckversuche mit Nahrung, Nahrungsaufbau, Entblockung/Dekanülierung
- Überprüfung der Effektivität von Haltungsänderungen, Modifikationen der Nahrung/Flüssigkeit

- Indikation weiterer diagnostischer Maßnahmen (Videofluoroskopie, pH-Metrie, Manometrie

- Indikationsstellung für die Funktionelle Therapie und deren Evaluation

- Indikationsstellung für prothetische und operative Therapiemaßnahmen.

Zur videoendoskopischen Untersuchung stehen folgende Methoden zur Verfügung:

- Transorale Untersuchung (Transorale Evaluation des Schluckvermögens =TOES) mit dem starren Lupenlaryngoskop [168]

- Transnasale Untersuchung mit einem flexiblen Endoskop gilt heute als Standarduntersuchung $[2,29,166]$

- Transstomale „retrograde“ Untersuchung bei Patienten mit Tracheostoma.

Jede der Methoden hat Vorteile und Einschränkungen. Die Untersuchungsprinzipien und Beurteilungskriterien sind bei allen gleich.

4.4.2.1 Evaluation des Schluckvermögens (TOES) mit starrer Endoskopie. Die lupenlaryngoskopische Untersuchung wird mit $70^{\circ}$ - oder $90^{\circ}$-Laryngoskop zur Beurteilung des Hypopharynx und Larynx und schluckrelevanter Funktionen (s. u.) durchgeführt.

Nach dem Entfernen des Laryngoskops erhält der Patient geeignete Substanzen zum Schlucken. Unmittelbar darauf erfolgt erneut eine Laryngoskopie. Jeweils nach der Modifikation der Nahrung und/oder der Durchführung von Reinigungs- und Schlucktechniken muss der Patient endoskopiert werden. Daher ist eine hohe Kooperationsbereitschaft erforderlich.

Die Vorteile der Untersuchung sind:

- Nach intensivmedizinischer Behandlung mit nasaler Intubation, Magensonden, Absaugmanövern, fürchten manche Patienten „wieder einen Schlauch in der Nase“ und tolerieren eher eine Lupenlaryngoskopie als eine transnasale Untersuchung.

- Während des Schluckens gibt es keine Interferenz durch das Endoskop.

- Es besteht keine zeitliche Limitierung. Therapeutische Interventionen wie oben angegeben (Änderung von Nahrung, Haltung, Schluckmodus, Reinigungstechniken) können beliebig oft wiederholt werden.

- Bei der Endoskopie hält der Untersucher die Zunge und kann damit den Kopf des Patienten leichter führen. Dies kann bei motorisch beeinträchtigten Patienten stabilisierend wirken.

- Die erwähnten Risiken der transnasalen Untersuchung werden vermieden (s. u.).

Die Nachteile der Untersuchung sind, dass folgende Phasen nicht beobachtet werden können:

- ein vorzeitiges Abgleiten von Material aus der Mundhöhle in den Pharynx oder Larynx (Leaking, prädeglutitive Aspiration)

- die beginnende Verschlussfunktion des Kehlkopfs

- die Wiederaufrichtung der Epiglottis nach Dorsalflexion während des Schluckreflexes.

Eine Schleimhautanästhesie ist obsolet, da sie die Schluckreflexauslösung beeinträchtigen würde. Daher kann es vorkommen, dass der Patient die Untersuchung wegen eines Würgereizes nicht toleriert. Die Mehrzahl dysphagischer Patienten weist jedoch erhebliche Sensibilitätsstörungen im Rachen auf. 
Obwohl die Überprüfung der Schluckfunktion bei der transoralen Untersuchung nur als „indirekte Beurteilung“ möglich ist, also postdeglutitiv, können Residuen, Penetration und Aspiration $[31,178]$ sowie die Effekte therapeutischer Interventionen und der Therapieevaluation ähnlich nachgewiesen werden wie bei der transnasalen Untersuchung.

4.4.2.2 Flexible Videoendoskopie des Schluckens. Die Prinzipien einer Untersuchung der Schluckfunktion mit der flexiblen Endoskopie und Videoaufzeichnung wurden 1988 von Langmore et al. [2] sowie 1991 von Bastian [166] unter dem Terminus „Flexible Endoscopic Evaluation of Swallowing (FEES)“ beschrieben und in den folgenden Jahren methodisch erweitert bzw. variiert. Die verschiedenen Möglichkeiten sind:

- Flexibel-endoskopische Schluckuntersuchung als Standard: Flexibles Rhinopharyngolaryngoskop, 2,3 oder 3,5 mm dick

- Flexible Endoscopic Evaluation of Swallowing with Sensory Testing (FEESST [180]): Flexibles Rhinopharyngolaryngoskop mit zusätzlichem Kanal für die Abgabe eines definierten Luftstromreizes zur Sensibilitätsprüfung

- Fiberskop (Bronchoskop) mit Absaugkanal zum Entfernen von Sekret und nichtabgeschlucktem Material sowie zur Flüssigkeitsabgabe; Eignung sowohl zur Sensibilitätsprüfung als auch zur Testung des Schluckvermögens; 4-6 mm dick [181-183]

- Transnasale Videopanendoskopie (ViP) für weitergehende Fragestellungen [184] s. Kap. 4.7 und Video.

Flexible endoskopische Schluckuntersuchung (FESU). Die Schluckuntersuchung wird mit dem flexiblen Rhinopharyngolaryngoskop gemacht. Die Bestimmung der Weite der Nasenhöhle erfolgt durch eine vordere Rhinoskopie. Gelegentlich kann eine Schleimhautabschwellung indiziert sein. Eine lokale Schleimhautanästhesie mittels Xylocain-Gel reduziert das Druckgefühl bzw. die Gefahr der Schmerzauslösung und verbessert die Gleitfähigkeit des Endoskops. Sie kann aber auch zu Hypersalivation, Sensibilitätsstörungen und Veränderungen des Schluckaktes führen.

Die Einführung erfolgt auf dem Nasenboden als anatomischer Gleitschiene. Zur Beobachtung der Velumhebung liegt die Spitze des Endoskops an der Grenze zwischen knöchernem und muskulärem Nasenboden im hinteren Drittel des unteren Nasengangs (Nasenhaupthöhle, entspricht Position 0 bei der ViP).

Zur Beobachtung von Vallecula, Zungengrund, Pharynx und Larynx wird das Endoskop in Höhe der Gaumenbögen dicht unterhalb der Uvula positioniert („obere Position“ oder „swallow position“, entspricht Position 1 der ViP). Die Lage der Endoskopspitze sollte während des Schluckreflexablaufs in dieser Höhe verbleiben, um die laryngealen Bewegungen nicht zu beeinträchtigen.

Zur eingehenden Betrachtung des Kehlkopfeingangs, der Stimmlippen und der Trachea kann die Endoskopspitze hinter den kranialen Epiglottisrand vorgeschoben werden („untere Position“ oder „postswallow position“, entspricht Position 2 der ViP) [30,171]. Nach Ablauf des Schluckvorgangs wird diese Position empfohlen, um eventuell penetriertes Material auch in geringer Menge zu erfassen und aspiriertes Material in Glottis und Trachea nachzuweisen (cave: vasovagaler Reflex), siehe auch S. 28: Videopanendoskopie und NBI.

Ist der Patient tracheotomiert, kann nach Entfernen der Kanüle die Glottis von unten beobachtet werden („retrograde Laryngoskopie“). Auf diese Weise lassen sich Tracheostoma und suprasto- male Trachea beurteilen. Spontane Speichelaspirationen, aber auch Aspirationen nach Gabe von Nahrung und Flüssigkeit sind erfassbar.

Vorteile der transnasalen Untersuchung:

- Anwendbar bei Patienten mit Bewusstseinseinschränkungen oder mangelnder Kopfkontrolle/Rumpfstabilität, z. B. auf Intensivstationen (siehe 3.7) [186,187]

- Geeignet für Kinder $[106,110,188,189]$ und Patienten mit begrenzter Kooperationsfähigkeit wie Patienten mit Demenzerkrankungen [181,190]

- Strukturen und Einzelfunktionen lassen sich beurteilen, die radiologisch nicht oder nur unzureichend zu erfassen sind, z. B. Entzündungen, Weichteilschwellungen, Defekte, Narben (insbesondere nach Tumortherapie) [191], Tonusveränderungen oder diskrete Bewegungsstörungen

- Aspirationen von Speichel sind nachweisbar, die beim Schlucken von Fremdsubstanzen, z. B. Kontrastmittel, nicht in Erscheinung treten [25 - 27,171,178]

- Beliebig oft wiederholbar und damit hervorragend zur Therapieevaluation geeignet

- Keine Strahlenexposition

- Die Untersuchung ist „vor Ort“ möglich, z. B. in allen Abteilungen eines Akutkrankenhauses oder in Reha-Einrichtungen sowie Alten- und Pflegeheimen [192-194]

- Nutzung als Biofeedback-Verfahren [195, 196]

- Geringere Kosten als radiologische Diagnostik.

Nachteile:

- Die orale Vorbereitungsphase ist nur bei Endoskopie mit Rückblick zu beobachten

- Während der pharyngealen Phase sind Zungengrund, Rachen und Kehlkopfstrukturen nur unmittelbar vor und nach der pharyngealen Phase erfassbar; während des reflektorischen Schluckablaufs kommt die Optik durch den „Mitnahmeeffekt“ des sich hebenden Velums und des sich kontrahierenden Pharynx meist in Kontakt mit der Rachenhinterwand. Das „white out“ [159] behindert bei der flexiblen Endoskopie die ausreichende Beurteilung der entscheidenden Komponenten Kehlkopfverschluss und Öffnung des Ösophagussphinkters

- Keine Beurteilung der Ösophagusfunktionen

- Kein Beurteilung der Menge aspirierten Materials

- Bei Patienten mit ausgeprägten motorischen Störungen wie Paresen, Ataxie, Tremor oder Dystonie kann es schwierig sein, das Endoskop zu positionieren und während der Schluckmanöver ruhig zu halten [189]

- Cave vasovagaler Reflex mit Hypotonie und Bradykardie, insbesondere bei Berührung der Epiglottis durch die Endoskopspitze, oder laryngealer Spasmus bei Berührung der Taschenfalten/Stimmlippen, wenn sich der Kehlkopf während des Schluckens hebt. Wegen der Seltenheit dieser Ereignisse gilt die Methode jedoch auch im ambulanten Bereich als sicher und wenig belastend für die Patienten [107,197,198].

Absaugmöglichkeit, Kreislaufbehandlung, Notfallkoffer, ggf. eine liegende Venenverweilkanüle sowie die Möglichkeit einer zeitnahen Notfallbronchoskopie sind für eine sichere Durchführung der flexiblen endoskopischen Schluckuntersuchung in schweren Fällen empfehlenswert [199].

Flexible Endoscopic Evaluation of Swallowing with Sensory Testing (FEESST). Durch ein speziell für diesen Zweck entwickeltes Rhinopharyngolaryngoskop mit einem Kanal (Fa. Pentax Precision Instrument, Orangeburg, New York) wird ein definierter Luftstrom auf den Kehlkopf abgegeben ( $50 \mathrm{~ms}$ mit einem 
Druck von 0-15 mmHg). Gewertet werden die Angaben der Patienten, ob und bei welcher Stärke sie den Stimulus spüren [180], und/oder die Auslösung des laryngealen Adduktionsreflexes (LAR, kurzer Glottisschluss als Reizantwort [200 - 203]. Die Untersuchung wird auch als Laryngopharyngeal Sensory Discrimination Threshold Testing (LPSDT) bezeichnet und kann zur Schwellenbestimmung dienen.

Pharyngolaryngoskopie mit bronchoskopischer Untersuchung. Mit einem transnasal eingeführten flexiblen Bronchoskop kann am Beginn oder während einer bronchoskopischen Untersuchung auch eine Untersuchung von Velum, Pharynx und Larynx sowie eine Schlucküberprüfung erfolgen. Dies bietet sich vor allem bei Patienten auf Intensivstationen und bei Patienten mit reduzierter Bewusstseinslage bzw. Kooperationsfähigkeit an.

$\mathrm{Zu}$ diesem Zweck kann über den Absaugkanal des Endoskops Flüssigkeit (Wasser, Kochsalzlösung, Lokalanästhetika) in den Pharynx verabreicht werden, und die Auslösung und der Ablauf des Schluckreflexes können unmittelbar beobachtet werden [181,182]. Vor Einbringen eines Lokalanästhetikums zur Bronchoskopie kann bei wachen Patienten eine Schluckuntersuchung mit Nahrung und Flüssigkeit erfolgen (FESU).

Nach der Passage der (im Anschluss an die Schluckprüfung normalerweise anästhesierten) Glottis ist die Beurteilung von Trachea und Bronchialsystem möglich und damit die Erfassung aspirierter Substanzen, die in die tiefen Luftwege gelangt sind.

\subsection{Untersuchungsmodalitäten und Beurteilungs-} kriterien der videoendoskopischen Untersuchung Trotz zahlreicher publizierter Untersuchungsprotokolle existiert bisher kein Goldstandard [159,163, 177, 204-206]. Die wichtigsten Beurteilungsparameter sind in $\odot$ Tab. 12 aufgeführt.

\subsection{Funktionsprüfungen}

Vor der Überprüfung mit Nahrung und Flüssigkeit erfolgt die Überprüfung schluckrelevanter Bewegungen von Pharynx und Larynx. Der laryngeale Verschluss kann gestört sein durch:

- Operationsdefekte

- Fixation des Krikoarytenoidgelenks oder andere mechanische Behinderungen, $z$. B. nach Intubation oder bei Sklerodermie, rheumatoider Arthritis

- Stimmlippenparesen oder Hyperkinesen (s. o)

- Reduzierung der Kraft der medialen laryngealen Kompression, z. B. bei Parkinson, Enzephalomyelitis disseminata, Atrophie der intrinsischen Larynxmuskulaur im Alter

- Unvollständige oder verspätete Auslösung des Schluckreflexes und/oder unvollständige oder aufgehobene Larynxelevation [117]

- Reduzierte oder aufgehobene Kehlkopfsensibilität [207].

\subsubsection{Prüfung des Verschlusses von Glottis und \\ Supraglottis}

- Phonation auf [e:]: In mittlerer Stimmlage möglichst entspannt über mehrere Sekunden anhalten; Beurteilung von Tonus und Beweglichkeit der Stimmlippen sowie Glottisschluss

- Mehrmalige Phonation [e] kurz hintereinander: Beurteilung der diadochokinetischen Beweglichkeit (bei spastischer Parese vermindert)

- Möglichst hohe, laute Phonation [iii:]: Überprüfung der Verlängerung des Stimmlippen und der Kehlkopfanhebung. Dabei erfolgt gleichzeitig die Überprüfung der Pharynxkontraktion, erkennbar an der lateralen Kontraktion der Pharynxmuskulatur und der Verengung der Recessus piriformes: „pharyngeal squeeze“ [208 - 210]

- Leichtes Atemanhalten: Glottisschluss

- Atemanhalten, leichtes und stärkeres Pressen: Aneinanderpressen und Anteriorbewegung der Aryknorpel, Schluss der Taschenfalten, Kontraktion der aryepiglottischen Falten, Dor-

Tab. 12 Beurteilungsparameter der pharyngolaryngoskopischen Untersuchung bei Dysphagie.

\section{Untersuchungsmodalitäten}

Ruhebeobachtung

Funktionsprüfungen

(ohne Nahrung)
Überprüfung der

Schluckfunktion mit Nahrung Überprüfung der Effektivität kompensatorischer Schlucktechniken und Reinigungsmanöver

\section{Beurteilungsparameter}

Strukturveränderungen: Defizite, Ödeme, Narben, Vorwölbungen der Rachenhinterwand, Entzündungszeichen (Reflux!) Stellungsveränderungen der Stimmlippen, der Glottisachse, Tonus-(Form-)Veränderungen, Hyperkinesen Beeinträchtigung respiratorischer Bewegungen Vorhandensein unwillkürlicher Bewegungen (Myoklonien, Tremor)

Zeichen des gestörten Schluckablaufs: Residuen von Speichel/Sekret und Penetration, Aspiration von Speichel/Sekret Beobachtung der Reaktion des Patienten auf: Residuen, Penetration und Aspiration (reflektorisches Husten, spontanes Räuspern)

Phonation auf [e:] (möglichst mit stroboskopischer Untersuchung):

Glottisschluss, Stimmlippentonus, Regularität, Ablauf der Schwingungen

repetitive Phonation he- he-he: diadochokinetische Bewegungen

Glissando: Kehlkopfhebung, Verlängerung der Stimmlippen

Phonation auf [iii:] so hoch und so laut wie möglich: Pharynxkontraktion

leichtes Atemanhalten: Glottisschluss

festes Atemanhalten und Pressen: Verschluss der supraglottischen Strukturen

willkürliches Husten: Glottisschluss, supraglottischer Verschluss mit Sprengung

bei Vorhandensein von Residuen, Penetration und Aspiration von Speichel/Sekret: Überprüfung der Fähigkeit und

Effektivität von Reinigungstechniken (Haltungsänderung, willkürliches Räuspern, Husten, Nachschlucken)

eventuell Sensibilitätsprüfung:

mit gebogenem Watteträger (bei transoraler Lupenlaryngoskopie) oder

Berührung mit dem flexiblen Endoskop (cave: vasovagaler Reflex, Laryngospasmus!)

Einbringen von Flüssigkeit durch Arbeitskanal des flexiblen Endoskops, wenn vorhanden

Wenn anamnestisch, klinisch und endoskopisch keine bedrohlichen Aspirationszeichen vorliegen und/oder der Patient zu effektivem willkürlichem Abhusten in der Lage ist

effektiveres Abschlucken

Vermeidung von Penetration und Aspiration

Verringerung/Elimination von Residuen, Penetration, Aspiration 
salwärtsneigung der Epiglottis [29,159,211,212]; letztere ist insbesondere bei Patienten ohne vollständigen Glottisschluss wichtig!

- Willkürliches Husten: Glottisschluss oder Taschenfaltenschluss mit adäquater Sprengung

- Bei Residuen/Penetration/Aspiration von Speichel oder Sekret: Überprüfung der Fähigkeit zur „Rachen- und Kehlkopfreinigung“ mittels Haltungsänderung, Räuspern, Husten und Nachschlucken oder notfalls Ausspucken.

\subsubsection{Sensibilitätsprüfung}

Die Sensibilität kann mit einem flexiblen Endoskop geprüft werden, indem durch den Absaugkanal Flüssigkeit in den Pharynx gespült, ein definierter Luftdruckstoßes (s.o.) gegeben oder Epiglottis, Taschenfalten, Aryknorpel, Stimmlippen mit der Endoskopspitze berührt werden. Während der Untersuchung mit dem starren Lupenlaryngoskop kann bei dringlicher Indikation eine Berührung des Kehlkopfeingangs mit einem gebogenen Watteträger erfolgen.

Eine pharyngolaryngeale Sensibilitätsstörung bedeutet ein hohes Aspirationsrisiko [117,203,213]. Bei ausgeprägtem Speichelüberlauf in den subglottischen Raum ohne kräftiges Husten muss vor der weiteren Diagnostik jede orale Nahrungszufuhr unterbleiben. Eine bereits liegende Trachealkanüle muss geblockt werden! Bei Patienten mit dieser Symptomatik lässt sich in manchen Fällen durch eine medikamentöse Speichelreduktion eine Tracheotomie vermeiden bzw. eine Dekanülierung beschleunigen.

\subsection{3 Überprüfung des Schluckvorgangs mit Nahrung}

Die Überprüfung des Schluckvorgangs mit Nahrung erfolgt, wenn keine bedrohliche Speichelaspiration vorliegt und der Patient zum effektiven willkürlichen Abhusten in der Lage ist, sodass er eventuell in die Trachea eingedrungenes Material wieder abhusten kann. Die Sicherheit des Patienten ist oberstes Gebot. Zur Untersuchung sollten zur Verfügung stehen [206]:

- Tasse/Wasserglas, Löffel, Strohhalm

- Eis (gefrorener Tee oder Fruchtsaft, als Stangeneis oder zerstoßen)

- Breiige Substanzen: wahlweise Götterspeise/Gemüsebrei/ Joghurt

- Flüssigkeit: flüssig (Wasser, Tee, Kaffee), sämig (Frucht- oder Gemüsesaft)

- Andickungsmittel zum Variieren der Flüssigkeit

- Weiche Kost: Kartoffeln, Gemüse, Weißbrot

- Feste Substanzen: Graubrot, Semmel, Keks, Kuchen

- Lebensmittelfarben, um verschiedene Substanzen zu markieren, falls diese nicht selbst eine von der Schleimhaut gut unterscheidbare Farbe aufweisen; manchmal ist ein Anfärben zur Differenzierung unterschiedlicher Konsistenzen hilfreich [214].

Patienten ohne bisherige orale Nahrungszufuhr erhalten in der Regel zunächst kleine Mengen (ca. $1 / 3 \mathrm{TL}$ ) geeister Götterspeise. Die halbfeste Konsistenz ist für die meisten Patienten leichter zu schlucken als Flüssigkeiten und feste Speisen, der Kältereiz provoziert evtl. eine bessere Schluckreflexauslösung und einen kräftigeren Schluckablauf. Götterspeise ist gleitfähig und verflüssigt sich bei Erwärmung im Körper. Sie kann daher bei Aspiration leicht abgehustet bzw. abgesaugt werden oder wird ohne größere Reizerscheinungen resorbiert. Durch grüne oder blaue Färbung lässt sie sich im Pharynx gut erkennen, und der Bolus lässt sich verfolgen (transnasale Untersuchung). Unmittelbar
Tab. 13 Sicher beobachtbare Symptome der pharyngolaryngoskopischen Untersuchung und ihr pathophysiologisches Korrelat bei Schluckstörungen [206].

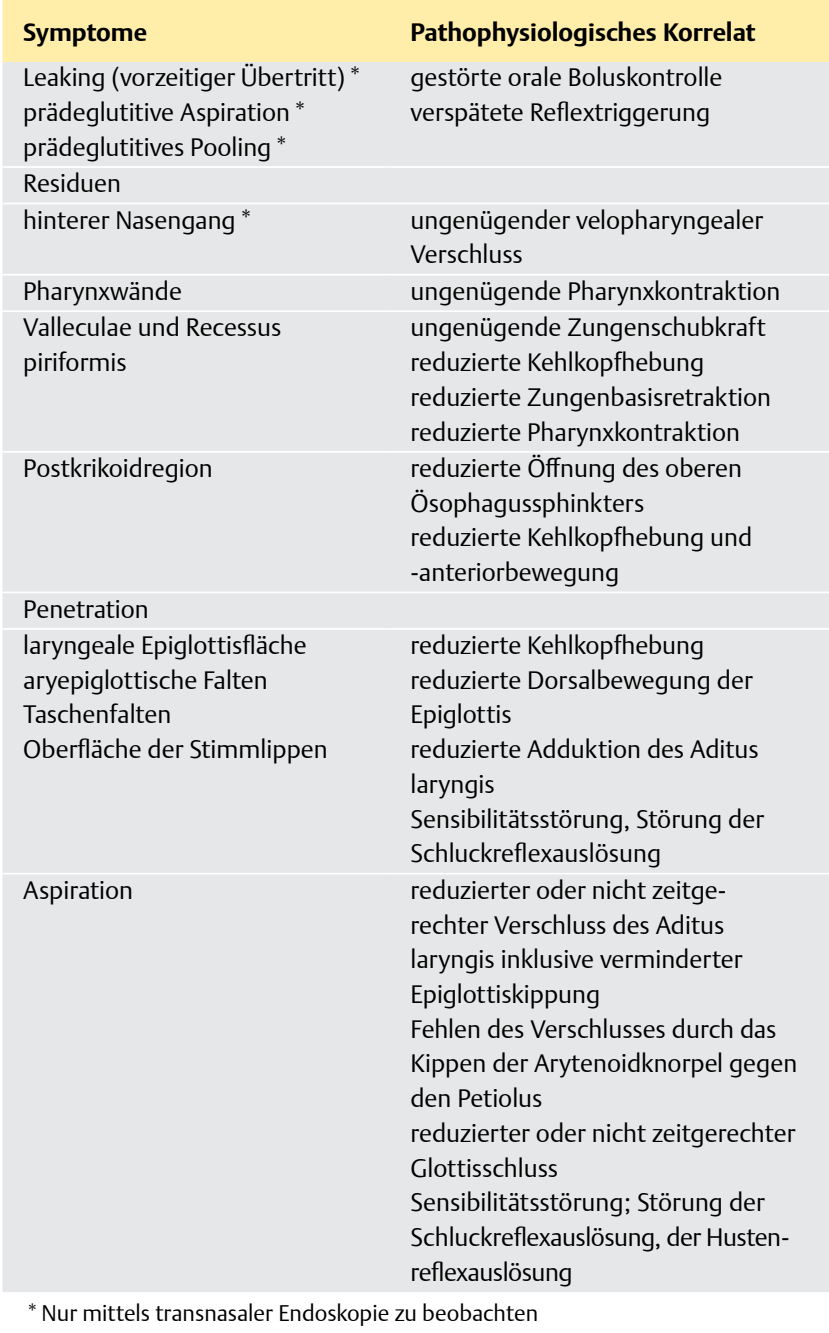

nach dem Schlucken lassen sich Residuen/Penetration/Aspiration identifizieren (transnasale und transorale Untersuchung). Bei sehr trockenen Schleimhäuten sind (z. B. nach Strahlentherapie) angedickte Flüssigkeit (z.B. Karottensaft oder Kaffee) oder feinst passierter Gemüsebrei oder Apfelmus günstiger.

Bei noch möglicher oraler Ernährung erfolgt die endoskopische Prüfung zuerst mit der für den Patienten am leichtesten zu schluckenden Konsistenz (in der Regel breiige Substanzen). Ist effektives Abhusten gewährleistet, kann Joghurt verwendet werden. Die leichte Säure verstärkt den sensorischen Input, Joghurt ist gleitfähig und gut sichtbar. Je nach bisherigen endoskopischen Auffälligkeiten wird daraufhin die Aufnahme von fester Kost (meist Butterbrot) und Flüssigkeiten (Kaffee, Tee) überprüft.

Die wichtigsten Ereignisse während der pharyngealen Phase, der Kehlkopfverschluss und die Öffnung des OÖS, sind mit der transnasalen endoskopischen Untersuchung nur dann erfassbar, wenn die Spitze des Endoskops in Position 3 im Vorausblick oder 4 im Rückwärtsblick liegt und das Bewegungsmuster des Patienten ausreichend Raum bietet. Daher ist auch die Erfassung einer intradeglutitiven Aspiration in der Regel nicht möglich und nur zu vermuten durch die Anfärbung der inneren Kehlkopfschleimhaut und der Subglottis als Zeichen eines unvollständigen Kehlkopfverschlusses. Die intradeglutitive Aspiration ist nur durch 
die radiologische Untersuchung bzw. eine retrograde Laryngoskopie durch ein Tracheostoma mit Sicherheit festzustellen.

Zeigen sich nach einem Schluck keine ausgeprägten Aspirationen, erhält der Patient eine etwas größere Menge $(1 \mathrm{TL}=\mathrm{ca}$. $3 \mathrm{ml}$ ), danach einen kleinen Esslöffel ( $=5 \mathrm{ml}$ ) des für ihn geeignetsten Lebensmittels. Anschließend wird die nächstschwierige Konsistenz geprüft.

Das Bolusvolumen wird versuchsweise vergrößert, auch wenn bei einer Menge von $1 \mathrm{ml}$ Aspirationszeichen auftreten, der Patient aber sicher abhusten kann. Die Effektivität des Schluckablaufs erhöht sich bei größerem Bolusvolumen und höherer Viskosität. Aus den beobachteten Symptomen und ihrem möglichen pathophysiologischen Korrelat lässt sich ein entsprechendes therapeutisches Konzept entwickeln ( $\bullet$ Tab. 13).

\subsection{4 Überprüfung der Effektivität therapeutischer} Manöver

Unmittelbar überprüfen lassen sich die Effekte von:

- Haltungsänderung

- Reinigungstechniken

- Schlucktechniken.

\subsubsection{Schweregrad der Aspiration und/oder der} Transporteffektivität von Substanzen

Ziele der Diagnostik von Patienten mit Dysphagie sind (einschließlich der Klärung der pathophysiologischen Ursachen der Störung):

1. Entscheidungen über die unmittelbare Sicherung der Atemwege: in schweren Fällen Tracheotomie und Einsatz geblockter Kanülen

2. Entscheidungen über den Ernährungsmodus: orale Nahrungs- und Flüssigkeitszufuhr möglich ohne/mit Einschränkungen, nasogastrale Sonde, PEG/PEJ.

Im Verlauf der Erkrankung ist zu beurteilen, ob eine Besserung durch eine funktionelle Therapie eintritt oder sogar chirurgische Eingriffe erwogen werden müssen [17]. Für diese weitreichenden Maßnahmen sind eingehende Bewertungen der klinischen, endoskopischen und/oder röntgenologischen Aspirationszeichen und des Schweregrades erforderlich. Verschiedene Einteilungen sind erarbeitet worden, die allerdings unterschiedliche Untersuchungsmodalitäten (klinische Beurteilung, Videoendoskopie, Videofluoroskopie) und verschiedene Kriterien bewerten (Auffälligkeiten bei der klinischen Untersuchung einschließlich dysarthrischer Symptome, Residuen, Aspirations-

Tab. 14 Deutsche Version der Penetrations-Aspirations-Skala nach Rosenbek [216].

1 Material dringt nicht in den Luftweg ein.

2 Material dringt in den Luftweg ein, verbleibt oberhalb der Stimmlippen und wird aus dem Luftweg ausgestoßen ${ }^{\text {a }}$.

3 Material dringt in den Luftweg ein, verbleibt oberhalb der Stimmlippen und wird nicht aus dem Luftweg ausgestoßen a.

4 Material dringt in den Luftweg ein, kontaktiert die Stimmlippen und wird aus dem Luftweg ausgestoßen.

5 Material dringt in den Luftweg ein, kontaktiert die Stimmlippen und wird nicht aus dem Luftweg ausgestoßen.

6 Material dringt in den Luftweg ein, passiert bis unter die Stimmlippen und wird in den Larynx hinein oder aus dem Luftweg ausgestoßen.

7 Material dringt in den Luftweg ein, passiert bis unter die Stimmlippen und wird nicht aus der Trachea ausgestoßen, trotz Bemühung.

8 Material dringt in den Luftweg ein, passiert bis unter die Stimmlippen, und es wird keine Bemühung zum Ausstoßen unternommen.

${ }^{a}$ Ausstoßen inkludiert: Husten, Räuspern und Schlucken (nach Rücksprache mit

J. Rosenbek) symptome von Speichel und/oder Nahrung, Fähigkeit zur Nahrungsaufnahme). Sie sind nur teilweise evaluiert und validiert.

\subsubsection{Penetration-Aspiration Scale (PAS)}

Die 8-stufige Einteilung stellten Rosenbek et al. 1996 vor [109]. Sie wurde für den radiologischen Befund und für die endoskopische Untersuchung gut evaluiert $[171,215]$. Sie hat sich sowohl für den Klinikgebrauch als auch zur Bewertung von Studien als geeignet erwiesen. Eine deutsche Version ( $\bullet$ Tab. 14) wurde 2014 von Hey et al. validiert [216].

Die PAS besitzt eine hohe Aussagekraft und eine gute diagnostische Treffsicherheit [171]. Sie bewertet im Wesentlichen das Vorhandensein und die Reaktion auf Material im Kehlkopf/der Trachea. Das Ausmaß der Residuen, also die Transporteffektivität, wird allerdings nicht berücksichtigt.

\subsubsection{Flexible endoskopische Evaluation der Dysphagie (FEED)}

Es gibt keine feste Vorschrift über den Untersuchungsablauf. Aus der Praxis der Untersuchungen in den Universitätsklinika Charité und Universitätsspital Zürich wurde ein streichen Bewertungsschema entwickelt, das auf der flexiblen endoskopischen Schluckuntersuchung beruht und zusätzlich Informationen liefert, die die Situation des Patienten beschreiben.

Zur Basisuntersuchung gehören folgende Angaben:

- Untersuchungsgrund

- Art der Ernährung, oral, parenteral, per Nasogastralsonde oder per PEG/PEJ

- Vorhandensein einer Trachealkanüle zum Zeitpunkt der Untersuchung, ja/nein

- Bisherige Therapie

- Situation bei der Untersuchung

- Körperkontrolle

- Allgemeinzustand

- Bewusstseinszustand

- Kooperativität

- Stimme, Sprache, Sprechen.

Untersuchungsbefunde:

- Fazialisfunktion

- orale Schleimhautbeschaffenheit

- Zungenmotilität und -kraft

- Drooling.

Bei der Untersuchung ohne Nahrung werden beschrieben:

- Schleimhautbeschaffenheit in Oro- und Hypopharynx

- velopharyngealer Verschluss

- Stimmlippenmotilität

- Schluss des Larynx bei Atemanhalten und Pressen: Stimmlippen, supraglottische Strukturen

- Sensibilität in Hypopharynx und Larynx

- Residuen/Penetration/Aspiration.

Bei der endoskopischen Schluckuntersuchung werden verschiedene Konsistenzen geprüft: z.B. Gel, Pudding, Wasser, Kuchen. Für jede Konsistenz wird beurteilt:

- Boluskontrolle

- Triggerung des Schluckreflexes

- Leaking

- Larynx-Hyoidelevation

- Einteilung nach der Penetrations-Aspirationsskala (PAS)

- Auswirkung von Manövern auf das Schluckvermögen (z.B. kräftiges Schlucken, Kopfneigung, Mendelsohn-Manöver, supraglottisches Schlucken, supersupraglottisches Schlucken, Kopfdrehung, Kopfkippung). 
Aus dem Ergebnis der Untersuchung werden die therapeutischen Konsequenzen abgeleitet:

- Funktionelle Dysphagietherapie

- fazio-orale Trakt-Therapie

- Sondenernährung (NG-Sonde, PEG)

- Empfehlungen zu diätetischen Maßnahmen

- bei Erfordernis chirurgische Therapie.

\subsubsection{FEES}

FEES ist eine Untersuchungs- und Bewertungsmethode [2,177]. FEES wird allgemein als Synonym für die flexible endoskopische Schluckuntersuchung genommen, mit festgelegten Untersuchungsschritten.

Die Reliabilität wurde mit der Einbeziehung von Videoaufzeichnungen und der Beachtung des umfassenden FEES-Protokolls gesteigert. Für evidenzbasierte Untersuchungen ist die Einheitlichkeit von Untersuchungsgang und Bewertung erforderlich [217]. Mithilfe einer Dokumentationssoftware haben Hey et al. die Auswertungszeit von 42 auf 18 min reduzieren können [218]. In der Praxis werden die Untersuchungsschritte nicht konsequent eingehalten. Das liegt zum einen an der Dauer der Untersuchung, zum anderen daran, dass nicht in jedem Fall das kom-

Tab. 15 Bogenhausener Dysphagiescore, BODS-1 (Speichelschlucken).

\begin{tabular}{|ll} 
Score & Charakteristika \\
1 & keine Trachealkanüle, effizientes Speichelschlucken
\end{tabular}

Tab. 16 Bogenhausener Dysphagiescore, BODS-2 (Nahrungsaufnahme).

\section{Score Charakteristika}

1 voll oral ohne Einschränkung

2 voll oral mit geringen Einschränkungen: Mehrere Nahrungskonsistenzen und mindestens eine Flüssigkeitskonsistenz ohne Kompensation oder Kompensation ohne Diäteinschränkung

3 voll oral mit mäßigen Einschränkungen: Mehrere Nahrungskonsistenzen und mindestens eine Flüssigkeitskonsistenz mit Kompensation

$4 \quad$ voll oral mit gravierenden Einschränkungen: Nur eine Nahrungskonsistenz und/oder eine angedickte Flüssigkeitskonsistenz mit oder ohne Kompensation

5 überwiegend oral: Mehr als die Hälfte des Tagesbedarfs, Restbedarf via Sonde/parenteral

6 Partiell oral: Mehr als 10 TL täglich bis zur Hälfte des Tagesbedarfs, Restbedarf via Sonde/parenteral

7 geringfügig oral: Weniger oder gleich $10 \mathrm{TL}$ täglich, Restbedarf via Sonde/parenteral

8 ausschließlich Sonde/parenteral plette Protokoll erstellt werden kann, weil die Patienten die Untersuchung nicht komplett durchstehen oder weil bezogen auf die Grunderkrankung die Symptome und Zeichen der Dysphagie nicht einheitlich sind.

\subsubsection{Bogenhausener Dysphagiescore (BODS)}

Der Bogenhausener Dysphagiescore (BODS) erfasst mit 2 verschiedenen Skalen die Fähigkeit des Speichelschluckens zum Schutz der tiefen Atemwege (BODS-1) und die Fähigkeit zur Nahrungsaufnahme (BODS-2). Die Skalen können einzeln oder als Summe zur Bestimmung des Schweregrades einer Dysphagie herangezogen werden und eignen sich sowohl für Patienten mit als auch ohne Trachealkanülen. Reliabilität und Inhaltsvalidität

Tab. 17 Einzelbewertung des Störungs-Schweregrads nach BODS-1 (Speichelschlucken) oder BODS-2 (Nahrungsaufnahme).

\begin{tabular}{|ll|}
\hline Score & Schweregrad \\
1 & keine Störung \\
\hline 2 & leichte Störung \\
\hline 3 & mäßiggradige Störung \\
\hline $4 / 5$ & mittelschwere Störung \\
$6 / 7$ & schwere Störung \\
\hline 8 & schwerste Störung \\
\hline
\end{tabular}

Tab. 18 Gesamtbewertung des Dysphagie-Schweregrads nach BODS-1 (Speichelschlucken) und BODS-2 (Nahrungsaufnahme).

$\begin{array}{ll}\text { Summenscore } & \begin{array}{l}\text { Schweregrad } \\ \text { keine Dysphagie }\end{array} \\ 2 & \text { leichte Dysphagie } \\ 3-4 & \text { mäßiggradige Dysphagie } \\ 5-6 & \text { mittelschwere Dysphagie } \\ 7-10 & \text { schwere Dysphagie } \\ 11-14 & \text { schwerste Dysphagie } \\ 15-16 & \end{array}$

Tab. 19 Schweregradeinteilung der Aspiration nach dem laryngoskopischen Befund.

\begin{tabular}{|c|c|}
\hline Schweregrad & Aspirationssymptomatik \\
\hline 0 & keine Aspiration \\
\hline I & gelegentliche Aspiration bei erhaltenem Hustenreflex \\
\hline II & $\begin{array}{l}\text { permanente Aspiration bei erhaltenem Hustenreflex } \\
\text { oder } \\
\text { gelegentliche Aspiration ohne Hustenreflex mit } \\
\text { gutem willkürlichem Abhusten }\end{array}$ \\
\hline III & $\begin{array}{l}\text { permanente Aspiration ohne Hustenreflex mit gutem } \\
\text { willkürlichem Abhusten }\end{array}$ \\
\hline IV & $\begin{array}{l}\text { permanente Aspiration ohne Hustenreflex, ohne } \\
\text { willkürliches effektives Abhusten }\end{array}$ \\
\hline
\end{tabular}

Tab. 20 Schweregrade der Aspiration nach dem radiologischen Befund.

\begin{tabular}{lll}
$\begin{array}{l}\text { Schwere- } \\
\text { grad }\end{array}$ & Aspiration & Hustenreflex \\
1 & $\begin{array}{l}\text { Aspiration des im Aditus und Ventriculus } \\
\text { laryngis retinierten Materials }\end{array}$ & erhalten \\
\hline 2 & $\begin{array}{ll}\text { Aspirationsvolumen von ca. } 10 \% \text { des Bolus } \\
\text { Aspiration } \leq 10 \% \text { des Bolus }\end{array}$ & $\begin{array}{l}\text { erhalten } \\
\text { reduziert } \\
\text { erhalten }\end{array}$ \\
\hline 4 & Aspiration $>10 \%$ des Bolus & fehlt \\
\hline
\end{tabular}


des BODS wurden nachgewiesen [219]. Tabelle und Handanweisung ( $\odot$ Tab. 15-18) zum BODS sind online verfügbar (www. elsevier.de).

Der Bogenhausener Dysphagiescore, BODS-2, bewertet die Möglichkeiten der Nahrungsaufnahme. Er ersetzt in konkreterer Form den „Food Oral Intake Score (FOIS)“ [220].

\subsubsection{Einteilung der Aspirationsschweregrade}

Nach dem videolaryngoskopischen Befund wurde 1996 eine Einteilung der Aspirationssymptomatik in die Schweregrade 0 - IV ( $\odot$ Tab. 19) angegeben [221].

Bei Schweregrad I und II können konservative Maßnahmen (Diätanpassung, funktionelle Schlucktherapie, Refluxprophylaxe) evtl. ausreichen. Bei Schweregrad III müssen weitreichendere Entscheidungen erwogen werden, vom Verbot oraler Nahrungszufuhr bis zu Tracheotomie und geblockter Kanüle. Mit dieser Einteilung werden das Vorliegen eines Hustenreflexes und die Fähigkeit zum willkürlichen Abhusten erfasst.

Da die Endoskopie nicht den gesamten Schluckvorgang und nur einen Teil der Ursachen der Störungen definieren kann, sollte insbesondere für die Anfangsdiagnostik einer klinisch relevanten Dysphagie komplementär eine Videofluoroskopie in Erwägung gezogen werden.

Nach Einführung der Hochgeschwindigkeitskinematografie in die Analyse des Schluckvorganges [222] galt die radiologische Untersuchung zunächst viele Jahre als Goldstandard [158]. Bei optimaler Erfassung des kompletten Schluckablaufs ist sie jedoch technisch aufwändig, mit Strahlenbelastung verbunden und nur bei kooperativen Patienten durchführbar. Nach dem radiologischen Befund wird häufig der Schweregrad der Aspiration nach Hannig et al. [222] eingeteilt ( $\bullet$ Tab. 20).
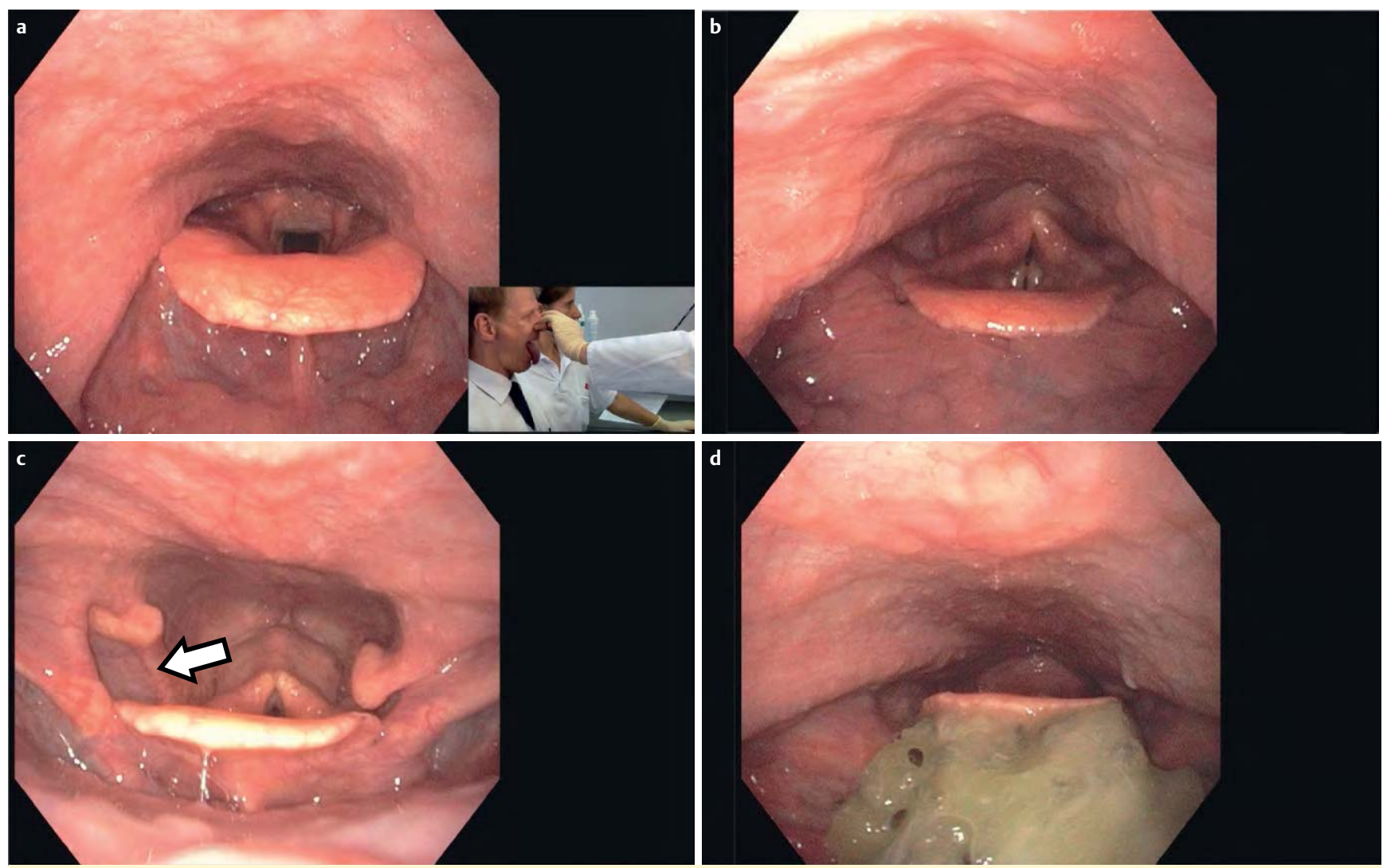

Der von Farneti 2008 vorgestellte „Pooling Score“ [223] beurteilt sowohl Residuen als auch Ansammlungen in Kehlkopfeingang und Glottis (also eigentlich Penetration und Aspiration) nach Lokalisation, Menge und „Management“ (Beeinflussbarkeit durch den Patienten), ist jedoch noch nicht ausreichend validiert.

Die Sekretbeurteilungsskala nach Murray et al. [27] wurde ebenfalls in der deutschen Übersetzung validiert und existiert in einer Lang- und Kurzversion [224].

Zahlreiche weitere Bewertungsbögen können für Erfordernisse bei verschiedenen Patientengruppen und unter klinischen oder wissenschaftlichen Aspekten herangezogen werden [103,204, 205, 220,223].

\subsection{Die transnasale}

\section{Videopanendoskopie (ViP)}

Die transnasale Videopanendoskopie wurde in Europa entwickelt und in den USA 1997 publiziert [184]. Sie kann angewendet werden, um neben Nase, Nasennebenhöhlen und Tuben auch Kehlkopf, Rachen, Speiseröhre und Magen unter Verwendung von ultradünnen

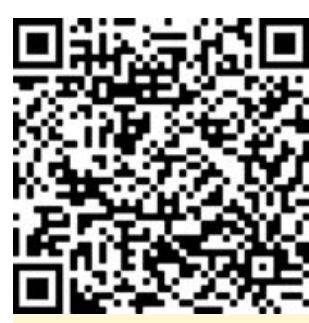

ViP-Video flexiblen Endoskopen zu untersuchen.

Messungen des Naseninnenraumes zeigten, dass flexible Endoskope mit einem Außendurchmesser von 4,0 mm bei $94 \%$ der Erwachsenen, zumindest auf einer Seite ohne ein Trauma oder Schmerzen durch die Nase geführt werden können. Normalerweise wird nur ein leichtes Druckgefühl wahrgenommen.

Die Länge des Endoskops sollte zwischen 60 und $100 \mathrm{~cm}$ betragen und die Spitze um bis zu $200{ }^{\circ}$ für eine Inversions-Ansicht gebogen werden können [280].

Abb. 19 Position 1 - Sicht über Larynx und Pharynx optisches Fenster (oF)= Höhe Gaumenbogen. a Bei herausgestreckter Zunge sind die Valleculae frei einzusehen. b Pharynx und Larynx bei Phonation „i“. c Der Valsalva-Versuch zeigt die Schildknorpelstrukturen und Pouch (Pfeil: muskelfreies Dreieck der Membrana thyreohyoidea). d Valleculae und Zungengrund bereiten den Kiwi-Bolus vor. 

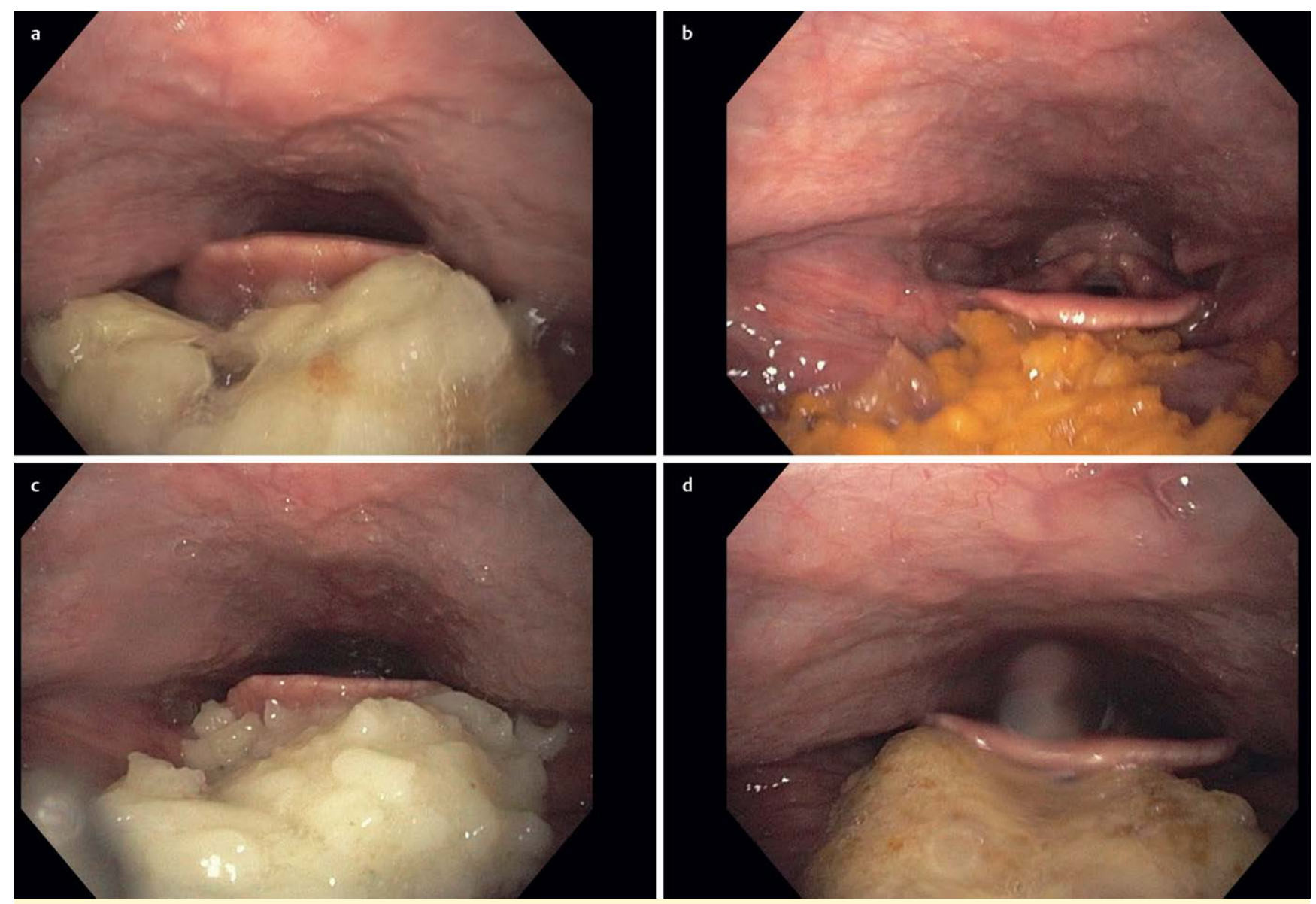

Abb. 20 Position 1 - of =Höhe Gaumenbogen, der Bolus wird über den Zungenrücken in den Valleculae gesammelt. a Vorbereitung Apfelschluckakt b Vorbereitung Karottenschluckakt c Vorbereitung Spaghettischluckakt d Vorbereitung halbfester Bolus.

Für die flexible transnasale Endoskopie sind die Standardpositionen P0-P6 bei Vorausblick und $180^{\circ}-200^{\circ}$ Rückblick definiert, um Vergleichbarkeit herzustellen [184,185]. Diese Positionen und das dazugehörige Blickfeld (optisches Fenster - oF) bei Blick voraus und Blick zurück sind ( $\odot$ Abb. 19 - 24):

- Position 0: das optische Fenster ist an der Grenze zwischen hartem und weichem Gaumen platziert. Blickfeld voraus: Nasenrachen, Tubeneingänge und Gaumensegel. Blickfeld zurück: Septum, Muscheln, Nasenboden und -dach der kontralateralen hinteren bis mittleren Nase.

- Position 1: das optische Fenster in Höhe der Gaumenbögen. Blickfeld voraus: Pharynx und Larynx mit Epiglottis, Blickfeld zurück: Vomer, Septum und Muscheln der hinteren Nase beidseits.

- Position 2: das optische Fenster ist hinter dem oberen Epiglottisrand platziert. Blickfeld voraus: Petiolus und Larynx, sowie Hypopharynxeingang beidseits mit Pharynxrückwand. Blickfeld zurück: Nasopharynx, Blick durch die Passavant'sche Enge in die Mundhöhle.

- Position 3: das optische Fenster liegt über der postkrikoidalen Region an der Pharynxrückwand. Blickfeld voraus: Arytenoide und Interarytenoidregion, Hypopharynxeingang beidseits. Blickfeld zurück: laryngeale Epiglottis, Gaumensegel, Zungengrund, Passavant'scher Ringwulst.

- Position 4: das optische Fenster ist in Höhe des oberen Ösophagussphinkters platziert. Blickfeld voraus: oberer - mittlerer Ösophagus mit Speisetransport und nachfolgender peristaltischer Welle. Blickfeld zurück: oberer Ösophagus mit OÖS.
- Position 5: das optische Fenster ist über der Ampulle oder 5 $\mathrm{cm}$ über dem UÖS platziert. Blickfeld voraus: die gesamte Zirkumferenz des UÖS. Blickfeld zurück: der untere und mittlere Ösophagus.

- Position 6: das optische Fenster ist unterhalb des UÖS platziert. Blickfeld voraus: Sicht auf den Mageninhalt oder auf die Korpus-Region. Blickfeld zurück: das Endoskop, das die Kardia passiert. Bei Rotation Sicht auf den Magenfundus.

Die FESU bezieht sich auf den Pharynx und gebraucht daher nur die Positionen 0 - 3.

Während der Durchführung gibt der Patient ein Fremdkörpergefühl in der Nase an. Abschwellende Nasentropfen mit lokaler Betäubung können diese Empfindungen minimieren. Eine mit Oxybuprocain-getränkte und ausgedrückte Watte wird in die Nase eingelegt und bewirkt nach ca. 5 min eine Oberflächenanästhesie. Die Berührung der Seitenwand des Nasenrachenraums, des Kehldeckels oder der Interarytenoidregion während der Durchführung der Endoskopie sollte außerhalb des Schluckaktes nach Möglichkeit vermieden werden, da hierdurch ein Würgereflex ausgelöst werden kann. Mit der Aufnahme von Speisen und Getränken in den Mund setzt eine physiologische Hypästhesie ein, die die Speisepassage in den Ösophagus ermöglicht.

Patienten können den Verlauf der Endoskopie am Monitor verfolgen. Damit hat der Untersucher die Möglichkeit, die endoskopischen Ergebnisse zu demonstrieren und Fragen der Patienten unmittelbar während der Untersuchung zu beantworten, wodurch das Vertrauensverhältnis zwischen Arzt und Patient gestärkt wird. 

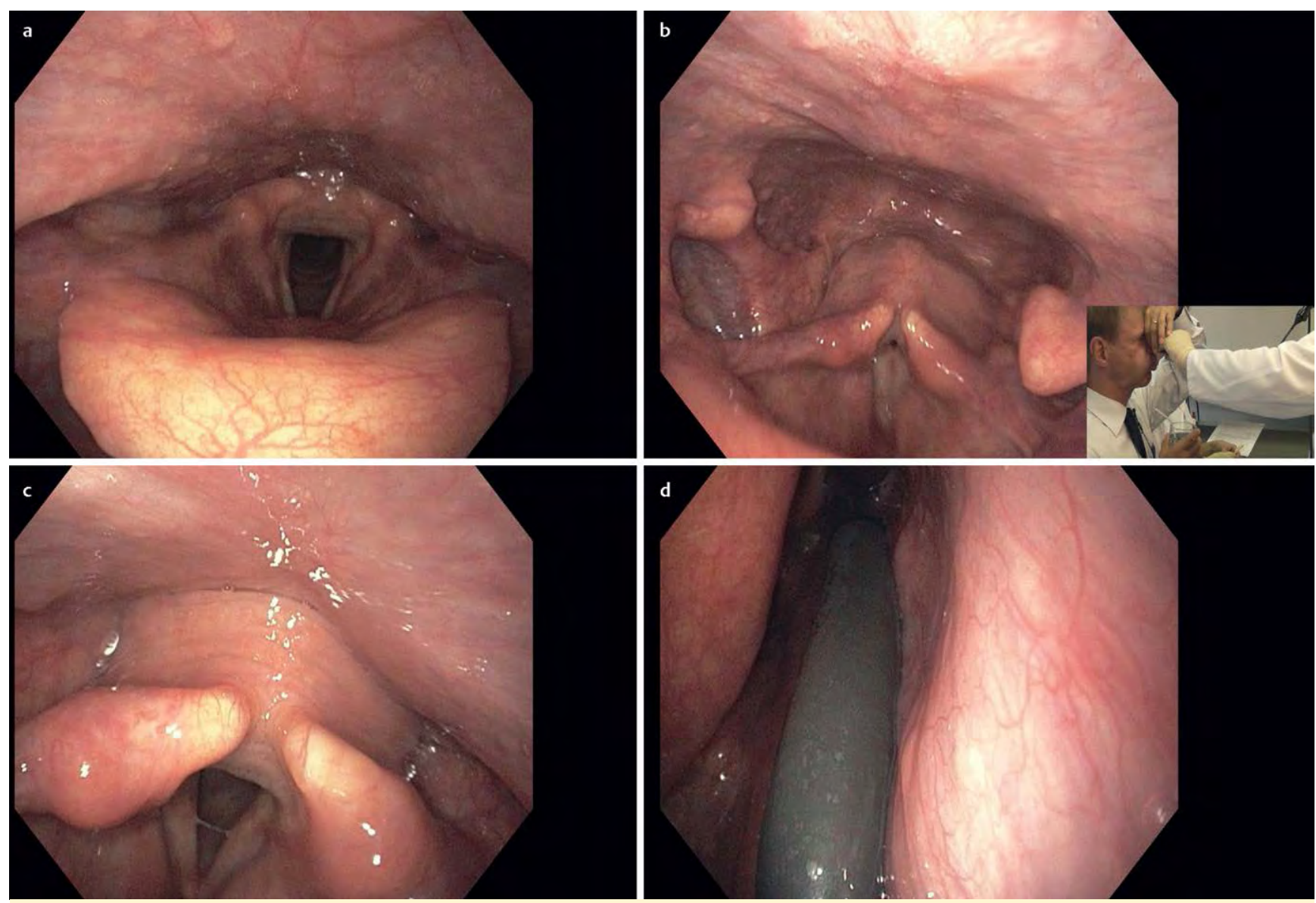

Abb. 21 a Position 2 - oF=Höhe Epiglottisoberrand, Sicht über Larynx und dorsalen Pharynx b Position 2 - oF=Valsalvamanöver c Position 3 - of =Vorausblick oberhalb der Interarytenoidregion d Position 3 - oF = Rückblick oberhalb der Interarytenoidregion.

Nase. Das Vorgehen bei der Passage der Nasenhöhle entspricht dem bei der FESU (s. 4.4.2.2).

Nasopharynx. Wenn die Endoskopspitze das dorsale Ende des Vomer erreicht, wird das Endoskop in die Nähe der Rachenhinterwand genau in der Mittellinie zwischen den Tori tubarii eingestellt (Position 0 - Grenze harter/weicher Gaumen). Dabei sollte der Patient ruhig durch die Nase atmen. Hierdurch entspannt sich das Gaumensegel und der Blick über den Oropharynx auf den Larynx und Hypopharynx wird frei.

Die unbedachte Berührung der lateralen Nasopharynxwand oder Tubenostien kann zum Schlucken und Würgen des Patienten führen. Bei Patienten mit Rhonchopathie vor allem im fortgeschrittenen Alter und bei Fettleibigkeit ist das Gaumensegel verdickt und verlängert. Gleichzeitig ist die Empfindlichkeit in diesem Bereich reduziert.

Oropharynx. Wenn sich die Spitze des Endoskops der dorsalen Rachenwand nähert, wird es mit dem Stellhebel in Richtung Oropharynx gebeugt und weiter vorgeschoben, bis der Kehlkopf sichtbar wird. Der Rachen und Kehlkopf werden von der Gaumenbogen-Aufsicht (Position 1: Höhe der Gaumenbögen, - Abb. 19, 20) beim Atmen, Husten, Essen, Trinken und Sprechen beurteilt.

Der Weg vom Gaumen bis zum Kehlkopf ist geräumig und erlaubt eine gute Beurteilung der anatomischen Strukturen. In diesem Zusammenhang ist es wichtig zu wissen, dass die Aufnahme von Nahrung und Flüssigkeit zu einer physiologischen Hypästhesie führt. Diese beginnt bspw. wenn die Lippen beim

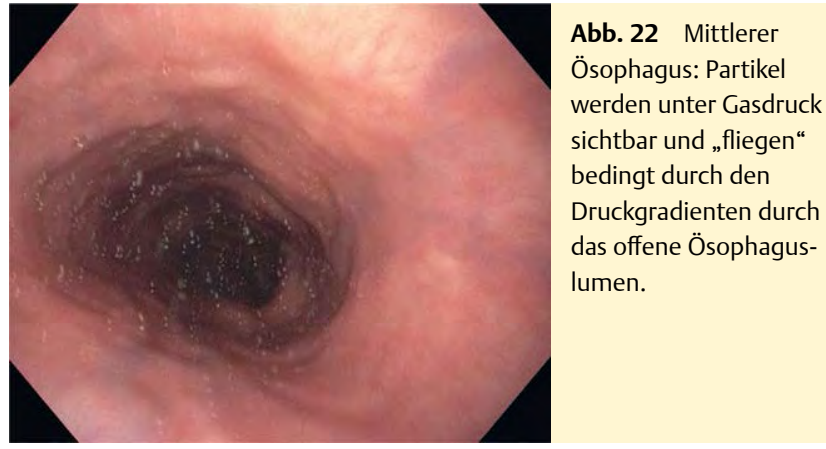

Trinken in Kontakt mit einem Strohhalm kommen. Dieser Prozess setzt sich fort, bis die Lippen den Strohhalm loslassen und sich schließlich der Pharynx wieder entspannt. Berührungen der Epiglottis mit dem Endoskop während dieses Vorgangs führen nicht zum Würgen oder Erbrechen (Position 2: Höhe des oberen Epiglottisrandes, $\odot$ Abb. 21).

Hypopharynx und oberer Ösophagussphinkter (OÖS). Vor der ersten Passage des oberen Ösophagussphinkters, muss getestet werden, ob der Patient in der Lage ist, kontinuierlich $\mathrm{zu}$ trinken. Der Patient sollte mindestens 2 Schlucke klaren Wassers ohne zu atmen zu sich nehmen können.

Um den oberen Ösophagussphinkter zu passieren, sollte die Spitze des Endoskops in der Nähe der Hypopharynxhinterwand auf der Höhe der Epiglottis platziert werden (Position 3: Nahe der Postkrikoidregion). Der/die Endoskopierende gibt dem Patienten die Anweisung, kontinuierlich zu trinken. Während des 

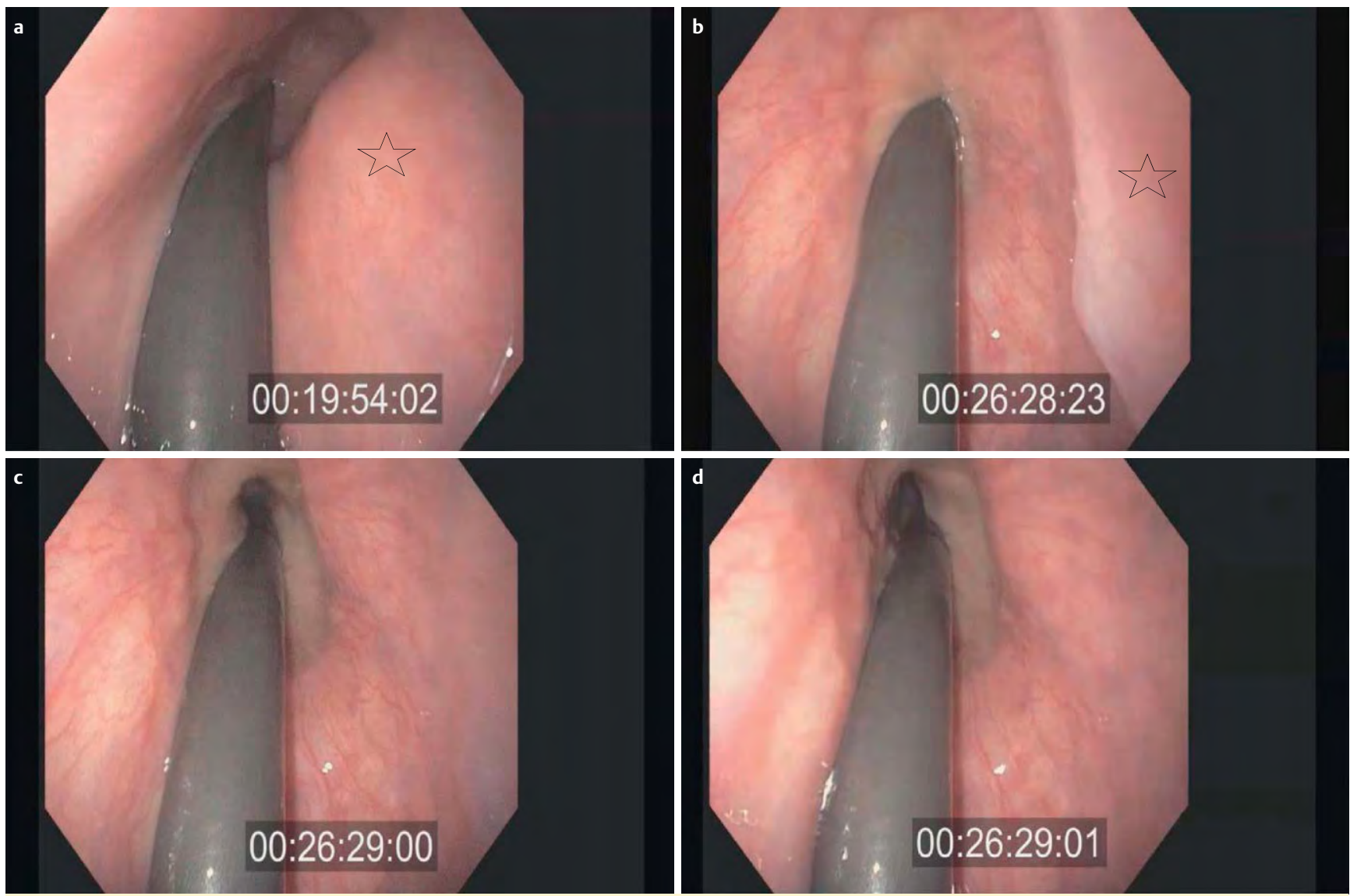

Abb. 23 Position 4 - oberer Ösophagussphinkter (OÖS) während des Aufstoßens im Rückblick (rechts zeichnet sich ein Wirbelkörper ( ${ }^{*}$ ) ab). a OÖS in Ruhe, ohne erhöhten Druck. b der untere Sphinkter war offen (Druckausgleich), der Raum unter dem Oös ist durch erhöhten Druck geweitet, die zirkulären Muskelfasern angespannt (gelb verfärbt). c die zirkulären Fasern öffnen zuerst (NB time code). d der M. cricopharyngeus ist offen, seine Konturen zeichnen sich ab (NB time code).

Schluckaktes wird das Endoskop durch den oberen Ösophagussphinkter geschoben und dessen Funktion aufgezeichnet. Danach ist der Weg in den Ösophagus frei und das Endoskop kann kontinuierlich weiter eingeführt werden. Kommt es vor dem Schluckakt zu einer unerwarteten Bewegung des Patienten, die eine Berührung der Epiglottis mit dem Endoskop zur Folge hat, kann es zum Auslösen des Würgereizes kommen. Damit wird die weitere endoskopische Beurteilbarkeit erschwert oder unmöglich. Beim Herausziehen des Endoskops aus dem Ösophagus sollte der Patient vor der erneuten Passage des oberen Ösophagussphinkter aufgefordert werden zu trinken. Dies hilft den Würgereflex beim Zurückziehen durch den OÖS zu überwinden. Alternativ kann das Endoskop durch den oberen Sphinkter sehr langsam und damit ohne zu reizen zurückgezogen werden.

Larynx. Wenn die Endoskopspitze den Pharynx erreicht, wird der Patient aufgefordert, das Trinken einzustellen. Nun wird die Spitze mit dem Hebel nach vorne bewegt. Das Bild ermöglicht die Sicht auf den Endolarynx aus nächster Nähe und vor allem auf die Stimmlippen während der Atmung und Phonation sowie die Trachea (Position 3) einschließlich Stroboskopie. Penetration und Aspiration sind hervorragend nachweisbar.

Ösophagus. Das Endoskop wird beim kontinuierlichen Schlucken in die Speiseröhre vorgeschoben, sodass der Patient die Bewegungen des Endoskops nicht wahrnimmt (○ Abb. 22). Wenn die gastro-ösophageale Übergangszone beim Wasserschlucken sichtbar wird, kann bei offenem unteren Sphinkter mithilfe des Schnüffeltest die Verschlussfähigkeit des unteren Ösophagussphinkters (UÖS) geprüft und beurteilt werden.

Schnüffeln der Nase bedeutet eine kurze und schnelle nasale Inspiration, die parallel mit der Kontraktion des Zwerchfells, des oberen Ösophagussphinkters und des Rhinosphinkters einhergeht. Bei offenem distalem Ösophagus kann die Höhe des Zwerchfells durch seine Kontraktion am sitzenden Patienten bestimmt werden.

Anschließend kann die Inversion des Endoskops im unteren Ösophagus etwa $5 \mathrm{~cm}$ über dem unteren Ösophagussphinkter durchgeführt werden. Der Radius des Endoskopes während der $200^{\circ}$-Flexion beträgt ca. $2,5-3,5 \mathrm{~cm}$. Durch die Verwendung dünner Endoskope mit einem kleinen Radius wird eine Irritation des Ösophagus vermieden.

Nach der Inversion wird das Endoskop mit rückwärtsgewandtem Blick zum oberen Ösophagussphinkter zurückgezogen (Position 4: oberer Ösophagussphinkter, $\odot$ Abb. 23).

Ist der Ösophagus z.B. bei einer Hiatushernie kollabiert, kann das Lumen durch fortlaufendes Trinken klaren Wassers offengehalten und beurteilt werden. Man kann zwischen Hypertonie, Hypotonie sowie normalem Muskeltonus des oberen Ösophagussphinkters unterscheiden. Beim Hypertonus kommt es zum Prolaps des postkrikoidalen kavernösen Schleimhautpolsters. Beim Hypotonus schließt die zirkuläre Muskulatur nicht, die kaudal an den M. cricopharyngeus anschließt. Ihre Verschlussfähigkeit kann mit dem Schnüffeltest geprüft werden. Sind beide Anteile des Sphinkters in Ihrer Verschlussfähigkeit einge- 

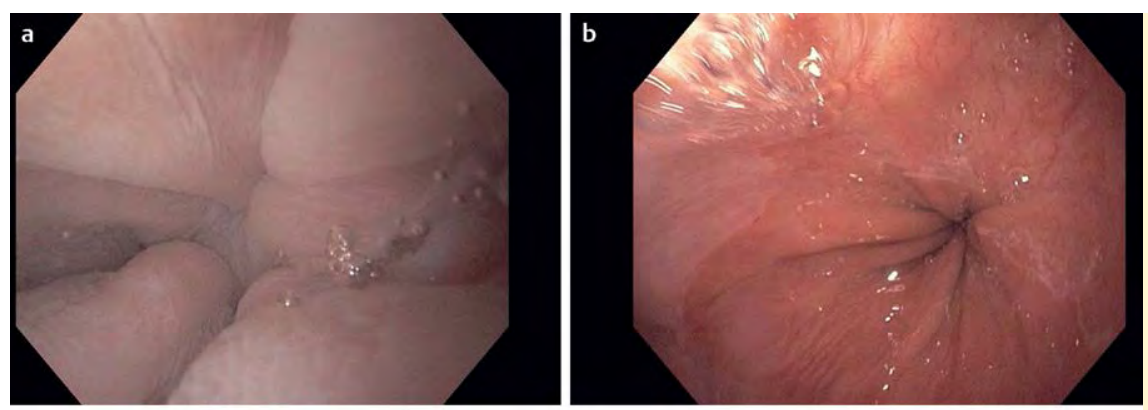

Abb. 24 Position 5 - vor dem unteren Ösophagussphinkter (UÖS) a Klares Wasser wird vor der Cardia gesammelt. b Die Cardia öffnet sich. Der ösophagogastrale Schleimhautübergang wird sichtbar. c Barrett-Schleimhaut im distalen Ösophagus.

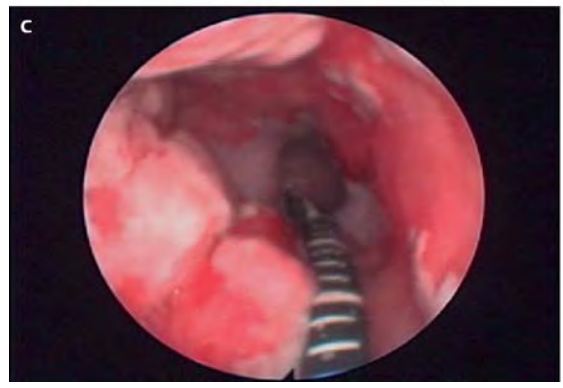

schränkt, ist der Blick auf die posteriore, kaudale Wand des Larynx frei. Der Kehlkopf sitzt wie der Verschlussstopfen einer geöffneten Sektflasche auf dem offenen Ösophaguseingang. Patienten klagen über permanentes Aufstoßen.

Im nächsten Schritt kontrolliert man die Funktion der Speiseröhre in Inversions-Ansicht beim Schlucken flüssiger und fester Nahrung. Hierbei wird das Endoskop wieder langsam nach distal in Richtung auf den unteren Ösophagussphinkter vorgeschoben (Position 5, ○ Abb. 24). Gleichzeitig kann der Bolustransport und die peristaltische Welle des Ösophagus im Rückblick und Vorausblick beobachtet werden.

Der untere Ösophagussphinkter (UÖS). Für die Passage des Endoskops durch den unteren Ösophagussphinkter in Inversions-Ansicht (Rückblick) bittet der Untersucher den Patienten mit dem Trinken aufzuhören. Beim Durchgang des invertierten Endoskops durch den Sphinkter kann der Untersucher einen Eindruck von dem Tonus und dem Schluss des unteren Ösophagussphinkters gewinnen. Neben der Anamnese und dem Lokalbefund ist der Widerstand des unteren Ösophagussphinkters entscheidend für die Beurteilung und Einleitung der weiteren Diagnostik, wie bspw. der hochauflösenden Manometrie.

Magen. Aus dem Magen wird in Inversions-Ansicht die Funktion des unteren Schließmuskels (Ösophagus und Magen-Übergangszone) beim sitzenden Patienten auch mit vollem Magen beobachtet (Position 6).

Oftmals wird die Gleitbewegung einer Hiatushernie erkennbar. Während der Aufnahme von flüssigen oder festen Nahrungsmitteln kann der Bolustransport von der Speiseröhre in den Magen gemeinsam mit der Funktion des unteren Ösophagussphinkters untersucht werden.

Der Weg zurück. Nach der Untersuchung des Magens und des unteren Ösophagussphinkters kann die Inversion aufgehoben und das Endoskop langsam zurückgezogen werden. Beim Herausziehen der Spitze des flexiblen Endoskopes aus der Speiseröhre sollte der Patient Wasser trinken. Die laryngeale Schleimhaut der Epiglottis sollte beim Zurückziehen außerhalb des Schluckens nicht berührt werden.
Rachen, Mundhöhle und Nase in Inversions-Ansicht. Mithilfe von 2 oder 3 Schluck Wasser kann der erfahrene Untersucher den oberen Schließmuskel mit dem invertierten Endoskop passieren. Die Postkrikoidregion wird ohne Probleme und Auslösen des Würgereflexes erreicht. Die Endoskopspitze wird an dem Übergang vom Oro- zum Hypopharynx (Position 3) positioniert und kann dann im Rückblick, falls erforderlich, nach oben und unten bewegt werden. Während dieser Manöver muss man darauf achten, dass der Patient ausreichend trinkt und isst, um die Unterstützung der natürlichen Hypästhesie zu nutzen und den Würgereflex im Rachen zu unterdrücken.

Von der Postkrikoidregion aus kann die Funktion der Gaumenbögen, des Zungengrundes und das Öffnen oder Schließen des Nasenrachenraums beobachtet werden. Es lassen sich sowohl die Bolussammlung und nachfolgend auch der Überlauf des Bolus als Auslöser für den Schluckvorgang nachweisen. Ebenso werden hyperplastische Tonsillen wie auch die Funktion der Zunge bei oralen und oropharyngealen Dysphagien im Zusammenspiel mit den Gaumenbögen sichtbar.

\subsection{Weitere diagnostische Verfahren}

Trotz der verbesserten endoskopischen Techniken sind Mundmotorik, Reflextriggerung, Öffnung des oberen Ösophagussphinkters und Ösophagusmotilität endoskopisch nicht ausreichend beobachtbar. Bei manchen strukturellen Veränderungen (z.B. Divertikel, HWS-Erkrankungen) können die damit verbundenen funktionellen Einschränkungen nicht sicher beurteilt werden, ebensowenig die Menge aspirierten Materials. In solchen Verdachtsfällen ist eine radiologische Untersuchung mit Darstellung des kompletten Schluckvorganges unverzichtbar. Verfahren mit einer schnellen Bildfolge sind erforderlich, damit auch kurze, diskrete Aspirationsepisoden erfasst werden:

- Hochgeschwindigkeitskinematografie: 50-200 Bilder pro Sekunde möglich, relativ hohe Strahlenbelastung

- Videofluoroskopie („Videofluoroscopic Swallowing Study“, VFSS): 25 - 30 Bilder pro Sekunde, heute noch gängiges Verfahren, als Digitale Fluoroskopie („Digital Fluoroscopic Swallowing Study“, DFSS) mit neuerer Bilderfassung weiterentwickelt

- Gepulste Durchleuchtung: dosissparend, hohe Bildqualität, Einspeisung in PACS Systeme möglich (picture archiving and 
communication system), Anpassung der Aufnahmefrequenz möglich, z. B. bei der Ösophaguspassage

Die Überprüfung erfolgt wie bei der endoskopischen Schluckuntersuchung mit unterschiedlichen Konsistenzen und Mengen (angereichert mit Kontrastmittel) und schließt die Effektivität von Reinigungs- und Schlucktechniken ein.

Eine pH-metrie ist unerlässlich bei Verdacht auf eine Refluxsymptomatik: zum Einen kann die Aspiration von Magensaft zu lebensbedrohlichen Lungenkomplikationen führen, saures Refluat im Ösophagus aber auch zu einer Verstärkung einer Öffnungsstörung des Oberen Ösophagussphinkters führen.

Die Manometrie des Pharynx und Ösophagus klärt Transportdefizite der Nahrung (,impaired propulsion“) und Störungen der Sphinkterfunktion, wie bei Patienten mit Myositis. Die Aussagekraft kann in Kombination mit der radiologischen Untersuchung („Radiomanometrie“) gesteigert werden.

Die Bronchoskopie wird bei Verdacht auf Lungenkomplikationen durch Aspiration diagnostisch eingesetzt zum Nachweis von Entzündungszeichen, von aspiriertem Material in Trachea und Bronchialbaum sowie zur Gewinnung von Material für die bakteriologische Testung. Therapeutisch werden aspirierte Fremdpartikel, Speichel, Sekret, Eiter, Nahrung mittels Bronchiallavage abgesaugt.

Bei weitergehenden Fragestellungen können EMG, Sonografie, MRT und Szintigrafie indiziert sein.

\section{Therapeutische Konsequenzen \\ $\nabla$}

\subsection{Konservative Dysphagietherapie}

Eine gezielte Therapie sollte möglichst früh beginnen. In der Akutphase zielt sie auf den Schutz vor Aspiration ab. In der Regel kommen erst adaptive und kompensatorische Maßnahmen, anschließend restituierende Verfahren zum Einsatz.

Die Therapiefrequenz richtet sich nach dem Zeitpunkt des Ereignisses, das die Schluckstörung ausgelöst hat. In der Akutphase sollte daher eine Schlucktherapie erfolgen, die die Grunderkrankung des Patienten berücksichtigt und je nach Belastbarkeit des Patienten längere oder kürzere Therapiesequenzen ein- oder mehrmals täglich (mindestens 5 mal pro Woche) beinhaltet.

In der postakuten Phase wird mindestens einmal täglich eine 45 minutige Einzeltherapie sowie Eigenübungsprogramme empfohlen. In der nachfolgenden ambulanten Therapie sollte 1 - 2-mal pro Woche für 45 min geübt werden, um bereits Erlerntes fortzusetzen, die Leistungen aufrecht zu erhalten und einen Transfer in den Alltag zu gewährleisten. Die Therapie endet, wenn das Therapieziel oder ein Leistungsplateau erreicht ist oder die Therapiemotivation fehlt.

Grundsätzlich werden 3 Ansätze unterschieden: Die funktionelle Schlucktherapie (FDT), die Fazit-orale Trakt-Therapie (F.O.T.T. ${ }^{\circledR}$ ) sowie die propriozeptive neuromuskuläre Faszilitation (PNF).

\subsubsection{Funktionelle Dysphagietherapie}

Der Begriff der funktionellen Dysphagietherapie wurde von Gudrun Bartholome [47] geprägt. Sie stellt ein funktions- und problemorientiertes Verfahren dar. Sie bedient sich restituierender (kausaler), kompensatorischer und adaptiver Methoden.

5.1.1.1 Restituierende (kausale) Methoden. Damit wird versucht, durch Stimulation sowie aktivierende und repetitive
Übungen ohne Nahrungsangebot die Schluckfunktion weitestgehend wiederherzustellen, zu restituieren. Elemente sind:

Thermische Stimulation. Die thermische Stimulation beruht auf der Annahme, dass die vorderen Gaumenbögen sensible Bereiche sind, die auf Temperaturreize reagieren, wodurch die Auslösung des Schluckreflexes erleichtert werden soll. Sie wird bei reduzierter oraler und pharyngealer Sensibilität indiziert.

Dazu wird eine Thermosonde in Eis abgekühlt. Die Gaumenbögen werden damit berührt oder die Patienten zerkauen einzelne Partikel zerstoßenes Eis, das die Basis der vorderen Gaumenbögen umspült. Dann werden die Patienten zum Schlucken aufgefordert.

Der beabsichtigte Effekt sind eine schnellere Auslösung des Schluckreflexes und die Verbesserung der pharyngealen Sensibilität. Die Wirkungsweise ist empirisch untersucht und nachgewiesen, wird dennoch kontrovers diskutiert [109,158].

Masako-Manöver. Die Indikation zur Übung dieses Manövers [225] ist bei einem schwachen Zungengrund und zu geringem Druckaufbau zu Beginn der pharyngealen Phase gegeben. Die Patienten werden mit folgenden Worten angeleitet: „Klemmen Sie Ihre Zunge zwischen die Zähne und schlucken Sie“. Der Effekt besteht in der Vorwölbung der Rachenhinterwand, die sich dem Zungengrund annähert und damit den Druckaufbau verbessert.

Shaker-Manöver. Bei einer Dysfunktion des oberen Ösophagussphinkters kann folgendes Manöver indiziert sein [226]:

"Legen Sie sich in Rückenlage, heben Sie $3 \times 1 \mathrm{~min}$ den Kopf (Schultern bleiben entspannt, dazwischen je 1 min Pause), heben Sie 30× den Kopf kurz.“ Diese Übung $3 \times$ am Tag wiederholen. Sie soll an 5 Tagen die Woche, 6 Wochen hintereinander durchgeführt werden. Als Effekt öffnet sich der OÖS sowohl in der Weite als auch der Dauer, der Bolusdruck erhöht sich, und die Kehlkopfhebung verbessert sich.

5.1.1.2 Kompensatorische Methoden. Durch Schlucktechniken und Haltungsänderungen mit Nahrungsangebot, auch wenn die physiologische Bewegungen nicht mehr oder nur teilweise wieder herstellbar sind, sollen Techniken erlernt werden, mit denen die Defizite bestmöglich kompensiert werden.

Der Patient muss im Falle von Hyperkinesen durch Erlernen kompensatorischer Strategien den Kehlkopfverschluss herbeiführen. Das kann dadurch wieder erreicht werden, dass er z. B. die Flüssigkeit in der Mundhöhle in kleine Schlückchen aufteilt, die Kopfhaltung verändert, feste Speisen gut kaut, kleinere Bolusmengen in den Valleculae sammelt und wegen der verlängerten Bolus-Peristaltik-Zeit (BPT) länger mit dem Beginn der Atmung wartet.

Mendelsohn-Manöver. Das Manöver wirkt sowohl restituierend als auch kompensatorisch [227]. Es ist bei Dysfunktion des OÖS, reduzierter Larynxelevation und reduzierter Zungenschubkraft indiziert.

Die Anleitung ist: „Halten Sie während des Schluckens den Kehlkopf 2-3 s oben. Drücken Sie den Zungenrücken gegen den Gaumen.“ - „Entspannen Sie, und schlucken Sie nach.“

Durch die Verlängerung des Schlucks wird die OÖS-Öffnung verlängert. Damit verbessert sich die pharyngeale Kontraktion. Retentionen verringern und die Kehlkopfhebung verlängert sich. 
Supraglottisches Schlucken. Bei verzögerter Reflexauslösung und bei unvollständigem Larynxverschluss wird supraglottisches Schlucken geübt $[158,228]$. Die Anweisungen an den Patienten sind: „Halten Sie die Luft an, schlucken Sie und räuspern/ husten Sie sofort danach ohne Zwischenatmung.“ - „Schlucken sie nach."

Mit dem supraglottischen Schlucken werden Retentionen und Penetrationen mobilisiert, entfernt und anschließend geschluckt.

Super-supraglottisches Schlucken. Das Manöver wird wie das supraglottische Schlucken durchgeführt. Zusätzlich zum Anhalten der Luft soll der Patient auch pressen. Damit wird der Verschluss von Glottis und Larynx stärker angeregt. Die tieferen Atemwege werden besser geschützt.

Haltungsänderungen. Mit Haltungsänderungen werden Schwerkraftverhältnisse durch Kopfneigungen und der Bolusweg durch Kopfdrehungen gezielt zur Regulierung des Bolustransportes modifiziert. Wegen individueller anatomische Verhältnisse soll die Wirksamkeit endoskopisch/radiologisch überprüft werden.

Kopfneigung nach vorne: chin tuck. Bei reduzierter oraler Boluskontrolle mit Leaking, einem verzögerten Schluckreflex oder reduzierter Zungengrundretraktion soll die Kopfneigung die orale Boluskontrolle unterstützen, und den Larynx durch stärkere Epiglottiskippung schützen [229].

Kopfneigung zur stärkeren Seite. Bei einseitiger oraler und pharyngealer Parese soll der Bolus sicher über die stärkere Seite geleitet werden.

Kopfdrehung zur betroffenen Seite. Bei einseitiger pharyngealer und laryngealer Parese soll sich die paretische Stimmlippe verkürzen und dadurch den Glottisschluss verbessern [230].

Kopfdrehung zur stärkeren Seite. Bei Stimmlippenparese soll die paretische Stimmlippe gedehnt werden und dadurch den Glottisschluss verbessern.

Beide Haltungsänderungen haben gleichen Effekt eines verbesserten Glottisschlusses. Die endoskopisch/radiologische Kontrolle ist wichtig zur Überprüfung der Wirksamkeit.

5.1.1.3 Adaptive Verfahren. Zu den adaptiven Verfahren zählen diätetische Maßnahmen wie das Andicken von Speisen oder bestimmte Kostformen und die Hilfsmittelversorgung wie ausgeschnittene Becher oder spezielles Besteck.

Zur Erleichterung der Nahrungsaufnahme und Verminderung des Aspirationsrisikos dienen:

- diätetische Veränderungen mit Anpassung von Viskosität, Temperatur und Geschmack unter Berücksichtigung der Pulmotoxizität

- eine geeignete Platzierung des Bolus auf die hintere Zunge und die gesunde Zungenseite

- spezielles Geschirr und Besteck. Weiterhin ist zu achten auf:

- optimale Körperhaltung

- im Falle von Nahrungsanreichung: Löffel von vorne führen

- ausreichend Zeit lassen

- ggf. manuelle Unterstützung für Mund- und Kieferschluss

- ggf. Schlucken durch streichende Bewegungen initiieren

- kleine Bissen

- gut kauen
- ggf. Nachschlucken

- den nächsten Bissen nehmen, wenn der Mund leer ist

- Mischkonsistenzen vermeiden

- Residuen über Kompensation verringern

- beim Essen nicht reden

ggf. Pausen einlegen

- bei Penetrationsverdacht die Phonationsprobe machen, um eine Kompensation zu erlernen.

Insgesamt wird die Wirksamkeit der funktionellen Schlucktherapie als gut eingeschätzt. So erreichen mehr als 50 \% sondenabhängiger Patienten nach Apoplex durch eine Schlucktherapie wieder eine vollständig orale Ernährung [104,231]. Bereits in der Akutphase des Schlaganfalls ist der Beginn einer intensiven Schlucktherapie wichtig. Bei „intensiver“ Schlucktherapie in der Akutphase $(5 \times /$ Woche) können sich nach 6 Monaten signifikant mehr Patienten als bei „Standard-Schlucktherapie“ ( $3 \times$ /Woche) wieder normal ernähren [232].

\subsubsection{Fazio-orale Trakt-Therapie (F.O.T.T. ${ }^{\circledR}$ )}

Die F.O.T.T. ${ }^{\circledR}$ stellt einen Behandlungsansatz zur Befunderhebung und Therapie neurogener Störungen, mimischer und oraler Bewegungen, des Schluckens, der Atmung, der Stimme und des Sprechens dar und wurde von Kay Coombes auf der Basis des Bobath-Konzepts initiiert [233]. Es stellt ein ganzheitliches Behandlungskonzept auf dem Boden interdisziplinärer Teamarbeit dar.

Ziel ist grundsätzlich das Wiederherstellen der gestörten Funktion (restituierendes Verfahren) sowie die Verminderung von Aspirationspneumonien, Kontrakturen, Hypersensibilität und die Verhinderung manifester abnormer Bewegungsmuster. Zentrale Elemente sind ganzkörperliche Tonusregulation, die Erarbeitung geeigneter Ausgangspositionen und die Koordination von Funktionen und Bewegungsabläufen. Neben der Mundhygiene werden auch die Fazilitation von Speichelschlucken sowie das Trachealkanülenmanagement integriert. Die Erarbeitung eines stabilen dynamischen Haltungshintergrundes und der Einsatz von taktil-kinästhetischen, visuellen, auditiven, olfaktorischen und gustatorischen Stimuli sind die Grundprinzipien. Mit ihnen werden gestörte Funktionen angebahnt und erlernt, Transferleistungen etabliert und die Verminderung oder das Verhindern von Aspirationspneumonien sowie der Manifestation abnormer Bewegungsmuster, Hypersensibilitäten und Kontrakturen angestrebt.

Bisher existieren keine randomisierten, kontrollierten Studien zur klinischen Wirksamkeit. Nach Frank et al. zeigte sich jedoch im Vergleich zur konventionellen Schlucktherapie eine signifikant kürzere Liegedauer der Trachealkanülen bei Patienten, die nach F.O.T.T. ${ }^{\circledR}$ behandelt wurden [234].

\subsubsection{Propriozeptive neuromuskuläre Faszilitation (PNF)} Die PNF wurde von Hermann Kabat und Margaret Knott entwickelt. Zusammenfassend stellt sie ein restituierendes und funktionelles Verfahren dar. Sensomotorische Bahnen sollen über Applizierung von speziellen Stimuli (thermale Reize, Druck, Dehnung, Widerstand) aktiviert werden. Das Ziel ist eine Tonisierung, Kräftigung oder auch die Tonusreduktion und damit eine Verbesserung der Bewegungsmöglichkeiten sowie die Verbesserung der Stabilität, die Ausführung einer aktiven Bewegung durch korrekten manuellen Kontakt und optimalen Widerstand, die Stimulierung von koordinierten Bewegungen bei korrekter Reizzugabe und die Verbesserung der Ausdauerfähigkeit 
Tab. 21 Empfehlungen zur Prävention medikamenteninduzierter Ösophagitiden.

- $100 \mathrm{ml}$ Wasser nach Medikamenteneinnahme

- vor dem Schlucken von Tabletten Wasser trinken

- Stehen oder sitzen 5-10 min nach Medikamenteneinnahme

- sorgfältiges Einhalten von Empfehlungen zu Medikamenteneinnahmen des Herstellers

- Tabletten mit Filmbeschichtung sinnvoll

- Auswählen der sichersten Darreichungsform (Liquid, Kapseln usw.

- Aufklärung der Patienten über Symptome bei medikamenteninduzierter Ösophagitis

Tab. 22 Medikamentöse Therapie bei Hypersalivation.

\begin{tabular}{ll}
$\begin{array}{l}\text { Atropin } \\
\text { Scopolamin }\end{array}$ & $\begin{array}{l}\text { häufig angewendet, wenig Studien } \\
\text { transdermal, p. o., über PEG }\end{array}$ \\
\hline $\begin{array}{l}\text { Ipratropiumbromid } \\
\text { Glycopyrrolat (Rubinol) }\end{array}$ & sublingual als Spray, uneinheitliche Studien \\
\hline $\begin{array}{l}\text { Trihexiphenylbenzhe- } \\
\text { xolhydrochlorid }\end{array}$ & $\begin{array}{l}\text { anticholinerge NW des Dystoniepräparates, } \\
\text { bei Kindern NW limitierend }\end{array}$ \\
Amitryptillin & geringe anticholinerge Wirkung des trizykl. \\
& Antidepressivums \\
Botulinumtoxine & Injektion in Speicheldrüsen
\end{tabular}

bzw. Verminderung von Ermüdungserscheinungen. Bisher fehlen Studien zur Wirksamkeit.

\subsection{Therapie bei medikamentenbedingter Dysphagie}

Es sollte kritisch hinterfragt werden, ob eine Mehrfachmedikation wirklich indiziert ist. Auf eine Dosisreduktion von Medikamenten sollte im Alter speziell bei degenerativen Gehirnveränderungen geachtet werden. Häufig wird eine „ordnungsgemäße“ Tabletteneinnahme automatisch stillschweigend vermutet, trotzdem sollte man darauf hinweisen, Medikamente mit ausreichend Flüssigkeit zu schlucken. Verschiedene Tabletten sind aufgrund von Größe und Oberfläche schwer einzunehmen. Dann bieten sich als Darreichungsform z. B. Hartkapseln an. Bei manifestem Verdacht auf eine OMIEI ist eine Ösophagogastroskopie ratsam (auch bei Kindern mit entsprechender Symptomatik). Je nach Befund ist eine PPI-Therapie sinnvoll. Weitere Alltagsempfehlungen zur Einnahme von Medikamenten, zeigt $\odot$ Tab. 21 [68].

\subsection{Therapie bei Hypersalivation und Xerostomie 5.3.1 Hypersalivation}

In erster Linie wird eine orofaziale Regulationstherapie bzw. funktionelle Dysphagietherapie zur Kontrolle des Speichels gemacht. In zweiter Linie setzt die medikamentöse Therapie an (๑ Tab. 22).

Gerber et al. [235] und Noonan et al. [236] kombinierten eine Exstirpation der Gll. submandibulares und eine beidseitige Ligatur der Stenongänge zur Reduktion der Speichelproduktion zur Vermeidung einer Aspirationspneumonie bei neurologisch beeinträchtigten Kindern. Dieses Vorgehen muss hinterfragt werden. Eine natürliche Funktion wird irreversibel ausgeschaltet, um eine Gefahr zu kontrollieren, ohne die Funktionsstörung selbst zu behandeln. Der Speichel reinigt die Mundhöhle, kontrolliert die Bakterienflora, vermeidet Karies, hält die Schleimhaut gleitfähig, unterstützt die Wundheilung, lindert den Schmerz, absorbiert und resorbiert Nahrungsbestandteile (Vit. B 12) wirkt prophylaktisch gegen Reflux, gelangt vor und mit der Speise in den Magen, fängt sie im Magen auf und hüllt sie mithilfe der Magensäure in ein klebriges Schaumnetzwerk [6].
Die Leitlinie zur Hypersalivation gibt eine Orientierung [237].

\subsubsection{Xerostomie}

Die klinische Untersuchung (trockene Lippen und Schleimhaut, atrophe Mukosa, gerötete furchige Zunge, kein Speichelsee Mundboden, eingedicktes Exprimat, Karies, Infektion), und die Diagnostik mittels Sonografie (ggf. Sialogafie, Sialometrie, Sialendoskopie, MRT ${ }^{99 \mathrm{~m}} \mathrm{Tc}$, ggf. Biopsie aus der Lippenschleimhaut) weist auf das Symptom einer Xerostomie hin. Eine Klärung der Ursache ist bei Ausschluss einer rheumatoiden oder medikamentösen Ätiologie nicht immer möglich. Eine Xerostomie kann nur symptomatisch therapiert werden:

- Ausreichend Flüssigkeit, Sialologa, Mundspüllösung

(Glycerin, schwarzer Tee), Speichelersatzmittel

- Medikamentös: Bromhexin, Anethol (Mukzinol), Parasympathikomimetika: Pilocarpin (Salagen), Cevimelinhydrochlorid (Evoxac), cave NW

- Prophylaxe für die Entwicklung der postradiogenen Xerostomie: Amifostin (Ethyol; Bindung alkylierender Substanzen und freier Radikale)

\subsection{Operative Therapie der Dysphagie}

Bei der Dysphagie mit chronischer Aspiration oder sogar rezidivierenden Aspirationspneumonien besteht ein Kampf zwischen Hunger, Durst und Husten [238]. Die Schwere der Bedrohung hängt von der Quantität der Aspiration ab. Ihre Beurteilung kann auch am Krankenbett ohne Sedierung durch die transnasale Funktionsendoskopie und/oder durch die Langzeitendoskopie ggf. bis zu mehreren Stunden erfolgen [239].

$\mathrm{Zu}$ den akuten lebenserhaltenden Interventionen zählen zum Schutz der Atemwege die Tracheotomie und zur Sicherung der Ernährung die nasogastrale Sonde oder die Anlage einer perkutanen endoskopischen Gastrostomie (PEG).

Folgende Gruppen von Störungen sind Grund für chirurgische Interventionen:

1. Akute Erkrankungen des motorischen Systems für reparative Chirurgie

2. Neurodegenerative Erkrankungen, solange sie langsam progredient verlaufen und damit nach einer chirurgischen Intervention realistische Chancen auf einen beschwerdearmen Lebensabschnitt bieten

3. Erkrankungen, die mit einer Sensibilitätsstörung bzw. -verlust einhergehen

4. Dysphagien als Folge therapeutischer Maßnahmen bei Tumorerkrankungen.

Die wichtigste Regel der Dysphagiechirurgie ist, bestehende Funktionen und Restfunktionen des Schluckens gemessen an der natürlichen Funktion zu erhalten. Bei der Vielzahl beteiligter Muskeln und Hirnnerven ist eine hohe diagnostische Präzision gefordert. Die Entscheidung für eine chirurgische Maßnahme wird im Falle einer neurologischen Grunderkrankung in interdisziplinärer Zusammenarbeit getroffen, mit dem Patienten sorgfältig besprochen und individuell angepasst.

Der Organerhalt bei tumorbedingten Störungen inklusive der damit verbundenen kritischen Risikoabwägung muss sinnvoll bleiben. Damit wird diese Chirurgie zu einer individualisierten Medizin, um Lebensqualität zu erhalten bzw. zu erreichen. Der Lernprozess anhand der Dokumentation von Langzeitverläufen durch Funktionsuntersuchungen ist integraler Bestandteil einer evidenzbasierten Behandlung.

Im Folgenden werden die chirurgisch-therapeutischen Schritte mit dysphagierelevanten Techniken kurz dargestellt. 


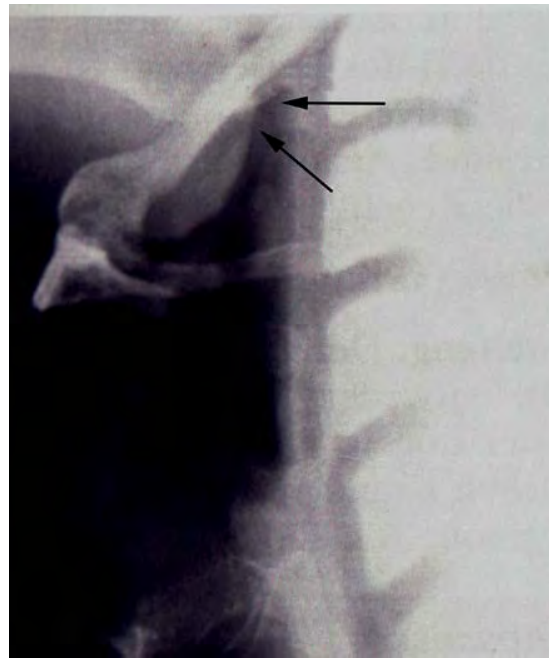

Abb. 25 Mangelnde Larynxelevation während des Schluckaktes beim Eagle-Syndrom. Pfeile zeigen auf die Epiglottis.

5.4.1 Chirurgische Therapie spezifischer Organstrukturen 5.4.1.1 Lippen-Kiefer-Gaumenspalte und velopharyngeale Insuffizienz und/oder Inkompetenz. Das Stillen von Kindern mit LKGS ist in der Regel unter Anleitung möglich. Das Trinken dauert länger. Lediglich bei durchgehenden LKGS sollte in den ersten Lebenstagen eine Gaumenplatte durch Kieferorthopäden angepasst werden, um die Abdichtung des Nasenrachenraumes gegen die Mundhöhle zu verbessern.

Die Zeitpunkte für alle weiteren chirurgischen Maßnahmen (z. B. Verschluss der Oberlippe, Palatoplastik [240], Schaffung eines ausreichend langen und funktionstüchtigen Velums [241], Augmentation der Oropharynxhinterwand mittels Fett [240,242]) werden interdisziplinär mit den Mund-KieferGesichtschirurgen abgestimmt.

5.4.1.2 Hyperplastische Zungengrundtonsillen und Epiglottiszysten. Als Folge eines Refluxes können Zungengrundtonsillen hyperplasieren. Wie man bei der Endoskopie im Rückblick aus der Position 4 (unterhalb des OÖS) sieht, taucht die Epiglottis nicht selten in den oberen Ösophagus ein. Partikel aus dem oberen Ösophagus werden von der pharyngealen Epiglottis wie mit einem Tennisschläger beim Zurückschnellen am Ende des Schluckaktes in den Pharynx befördert und am Zungengrund „abgewischt“. Bleiben die Valleculae unter der Zungengrundtonsille bei Herausstrecken der Zunge verborgen, liegt eine Hyperplasie vor. Nur wenn sie zu Dysphagiebeschwerden wie störendes Globusgefühl, Räusperzwang und Schluckstörung führt, sollte die Zungengrundtonsille laserchirurgisch unter strikter Schonung der Muskulatur entfernt werden [243]. Hierfür stehen moderne Verfahren wie Laserchirurgie, TORS und Radiofrequenzchirurgie zur Verfügung. Epiglottiszysten können durch eine Marsupialisation oder komplette Exzision im Rahmen der Mikrolaryngoskopie abgetragen werden.

Besteht der Verdacht auf einen Tumor, ist eine Biopsie erforderlich. Eine Zungengrundstruma ist auszuschließen. Eine Kontrolle der Funktion des OÖS ggf. auch eine pH-Messung im Rachen kann bei der Entscheidung für eine Intervention hilfreich sein.

5.4.1.3 Eagle-Syndrom. Das Eagle-Syndrom ist eine seltene Erkrankung, die durch eine Elongation des Proc. styloideus oder die Kalzifikation bzw. Ossifikation des Lig. stylohyoideum hervorgerufen wird [244]. Hierdurch wird die Elevation von Hyoid und Larynx behindert ( $\bullet$ Abb. 25).
Die Patienten beklagen unklare Halsschmerzen, Schluckbeschwerden, Fremdkörper- und Globusgefühl. Die Symptomatik kann durch den Druck auf die Hirnnerven V, IX, X sowie die A. carotis communis bedingt sein. Die chirurgische Therapie besteht in der transoralen oder transzervikalen Resektion des Proc. styloideus bzw. des verknöcherten Ligaments ggf. mit Resektion des kleinen Zungenbeinhorns [245].

5.4.1.4 Zervikogene Dysphagie. Die zervikogene Dysphagie ist meistens funktionell bedingt [46](Seifert). Daher kann nach der Endoskopie der Luft- und Speisewege sowie der manuellen und radiologischen Untersuchung der HWS die Diagnose einer FSH gestellt werden. Diese wird anschließend durch Mobilisation oder auch Manipulation der HWS im Segment (in der Regel C3/4) als Reflextherapieverfahren gelöst. Dies kann mit einer lokalen Lidocain-Injektion am druckdolenten Ansatz der infrahyoidalen Muskeln am Zungenbein oder auch einer Akupunkturbehandlung kombiniert werden. Nachfolgende Physiotherapie sollte diese Behandlung ergänzen. Die partielle oder auch vollständige Beseitigung der Symptome durch die manualtherapeutische Behandlung ist der klinische Beweis für das Vorliegen einer FSH.

Bei Patienten mit einer organisch bedingten zervikogenen Dysphagie kann in vielen Fällen durch die Modifikation der Nahrungskonsistenz eine Besserung der Beschwerden erzielt werden. Üblicherweise bleibt der M. Forestier asymptomatisch. Daher stellen symptomatische zervikale Osteophyten eine seltene Erkrankung dar, die vor allem Männer betrifft. Außer in besonderen Notfällen, sollte der konservativen Behandlung zunächst der Vorzug gegeben werden. Die Therapie erfolgt symptombezogen, wobei Analgetika, Antiphlogistika, Muskelrelaxantien, Protonen-Pumpen-Inhibitoren und physikalische Therapie zum Einsatz kommen. Vor allem Physiotherapie und gezielte Schlucktherapie können zur Verbesserung oder Vermeidung der Dysphagie erfolgreich angewandt werden [246]. Bei Versagen der konservativen Maßnahmen beseitigt bzw. lindert die Entfernung der Osteophyten bei strenger Indikationsstellung die Symptome, wenn sie die Ursache für den Gewichtsverlust sind [50]. Bessern sich nach den beschriebenen Therapieformen die Symptome nicht, muss nach vollständiger Diagnostik auch die Anlage einer perkutanen endoskopischen Gastrostomie erwogen werden.

5.4.1.5 Verstärkung des muskelfreien Dreiecks der Membrana thyrohyoidea. Aussackungen entstehen bevorzugt im muskelfreien Dreieck der Membrana thyrohyoidea dort, wo der N. laryngeus superior mit den Gefäßen in den Larynx eintreten (siehe $\odot$ Abb. 19c und 21b). Dieses Dreieck grenzt kranial an das Zungenbein, vorne und kaudal an den M. thyrohyoideus und den M. omohyoideus und kaudal und hinten an den M. constrictor pharyngis inferior. Eine Retention von Teilen des Bolus oder von Flüssigkeit in der Aussackung kann zu einer postdeglutitiven Aspiration führen, wenn der Larynxschluss gestört ist. Wenn nicht, kann der Patient durch das supraglottische oder supra-supraglottische Schlucken den Pharynx reinigen.

Vor der chirurgischen Behandlung einer großen Aussackung mit Aspiration ist es notwendig eine Vagusparese oder -paralyse auszuschließen. Mit einer spontanen Heilung des Nervs verschwindet wahrscheinlich auch die Aussackung spontan. Die Dysfunktion des $\mathrm{N}$. vagus wird videoendoskopisch, videofluoroskopisch, durch high-resolution manometry oder durch Elektromyografie des $\mathrm{M}$. cricothyroideus, versorgt durch den $\mathrm{N}$. laryngeus superior, bestätigt. Liegt keine Vagusparese vor, erfolgt die operative Versorgung in lokaler Betäubung [36]. Der 

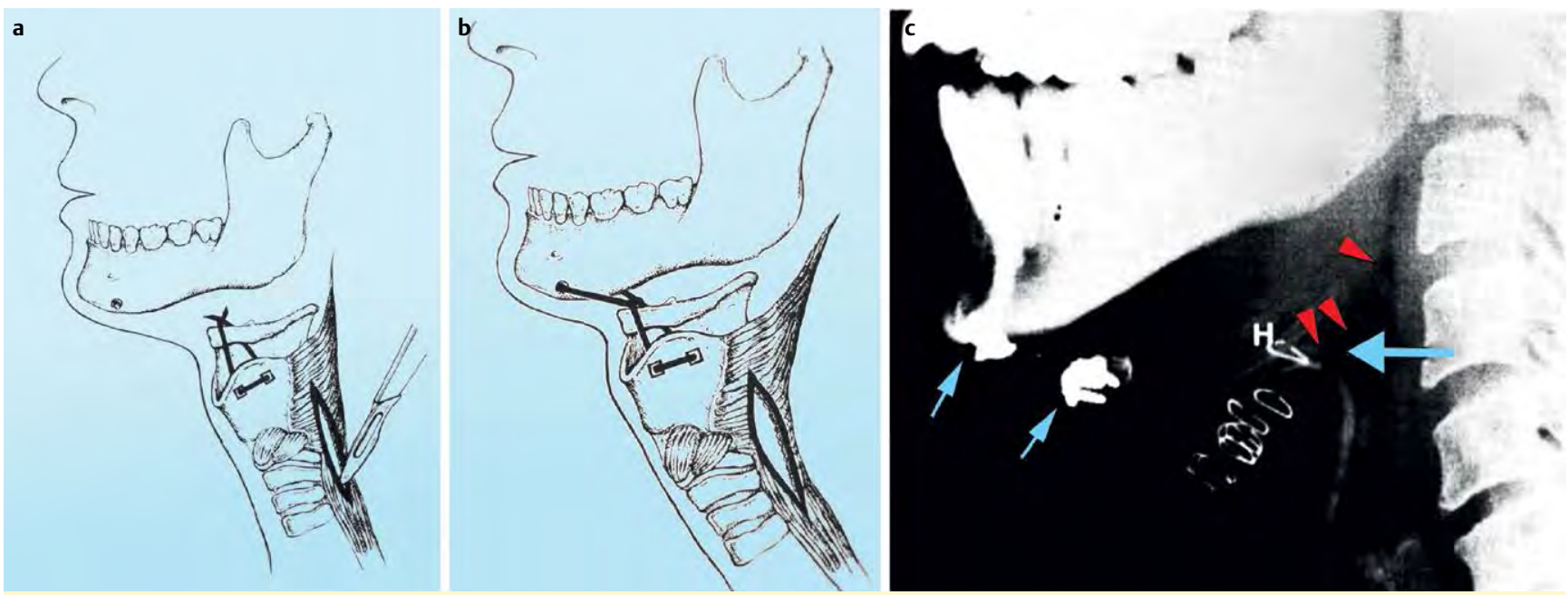

Abb. 26 a Ausdehnung der transzervikalen Myotomie des M. cricopharyngeus (ca. $4 \mathrm{~cm}$ ) unter Schonung des N. laryngeus inferior mit Laryngohyoidopexie. Die Myotomie sollte etwas nach kranial und kaudal verlängert werden. Alle Muskelfasern sind im Bereich der Myotomie zu durchtrennen (b). b Zusätzlich zur Myotomie wird eine Suspensionsnaht durch den Unterkiefer angelegt, um den Laryngohyoid-Komplex zur Protektion unter den Zungengrund zu ziehen. c 43 Jahre alter Patient nach Op. eines Ependymoms des 4. Ventrikels. Nach der Myotomie wurde eine Laryngo-Hyoido-Mentopexie durchgeführt. kleine, blaue Pfeile: Osteosytheseplättchen der Hyoido-Mentopexie. Blauer Pfeil: Vestibulum laryngis unter dem Schutz des Zungengrundes. rote Pfeilköpfe: Weichteilschatten des Zungengrundes. $\mathrm{H}=$ Hyoid.

Hautschnitt wird in Höhe der Aussackung entlang der Hautspannungslinien ausgeführt. Unter Schonung des oberen laryngealen Gefäßnervenbündels wird die Membrana thyrohyoidea dargestellt. Die Aussackung wird während des Valsalva-Manövers sichtbar. Die schwache Wand kann durch einen frei transplantierten, entsprechend zugeschnittenen Septum- oder Conchaknorpel des Patienten und/oder durch die Transposition des M. omohyoideus verstärkt werden:

1. Die Anheftung des M. omohyoideus am Hyoid wird durchtrennt und nach lateral auf das Cornu majus zur Abdeckung der Aussackung verlagert. Zusätzlich wird der Muskel durch eine Naht mit der Cartilago thyroidea, dem M. constrictor pharyngis und dem M. thyrohyoideus verbunden [247].

2. Der Knorpel des Patienten vom Septum oder von der Concha kann alternativ über die Aussackung gelegt und wie oben beschrieben fixiert werden. Beide Techniken können kombiniert werden.

Zur Ruhigstellung wird für eine Woche eine nasogastrale Sonde gelegt. Um Larynxbewegungen zu reduzieren, wird der Patient gebeten, wenig zu sprechen und zu schlucken und ggf. den Speichel auszuspucken.

5.4.1.6 Positionierung der Laryngohyoid-Einheit. Die Indikation zur Chirurgie hängt von der Funktion der Thyrohyoidmuskeln, der Funktion der vorderen Suprahyoidmuskeln, der Sensibilität der Triggerzonen für den Schluckakt sowie vom Zustand der Mukosa ab. Mit einer Verlagerung von Larynx und Hyoid aus dem Speiseweg nach ventral unter den Schutz des Zungengrundes kann die Aspiration reduziert werden.

Verschiedene laryngeale Suspensionstechniken wurden beschrieben [248], um das Aspirationsproblem nach tumorchirurgischen Eingriffen zu vermindern. Sie verwendeten Nahttechniken, um den Schildknorpel nach vorn zu verlagern und der Mandibula zu nähern. Hillel und Goode hängten den Larynx lateral mit Nähten auf, um das Aspirationsproblem nach Zungengrundresektion zu lösen [249]. Herrmann beschrieb eine Suspensionstechnik zum Kinn durch Verwendung von Fascia lata nach Laryngohyoidopexie und Myotomie des oberen Speiseröhrens- phinkters bei schweren neurologischen Schluckstörungen $[36,247]$. Diese Technik achtet auf die richtige Position der laryngohyoidalen Einheit im Pharynx, berücksichtigt die Funktion der suprahyoidalen Muskulatur, und die Elevation der Epiglottis, um den Larynxeingang zu schützen.

5.4.1.7 Laryngohyoidopexie. Zu Beginn des Eingriffs wird eine PEG gelegt (Kapitel „Perkutane endoskopische Gastrostomie (PEG)“), um postoperativ das Operationsgebiet möglichst ruhig zu stellen und die Nahrung problemarm zuzuführen. Das bestehende Tracheostoma wird umschnitten und ein kranial gestielter Hautlappen präpariert. Sollte kein Tracheostoma angelegt sein, ist dies der erste operative Schritt. Die Schilddrüse wird dargestellt, der Isthmus durchtrennt und die Trachea freigelegt. Trachea und Pharynx werden von der Schilddrüse gelöst und mobilisiert. Die Nn. recurrentes werden geschont.

Danach folgt:

1. Die Myotomie des M. cricopharyngeus ( siehe Kapitel „Myotomie des oberen Speiseröhrensphinkters“)

2. Durchtrennung der infrahyoidalen Muskulatur: Um den funktionsgestörten Kehlkopf aus dem Zentrum der Schluckstraße herauszunehmen und unter den Schutz des Zungengrundes zu verlagern, wird die infrahyoidale Muskulatur mit Ausnahme des M. thyrohyoideus durchtrennt [250]. Die Einheit von Kehlkopf und Hyoid kann nun in die gewünschte Position gebracht werden.

3. Die Laryngohyoidopexie und die Suspension der laryngohyoidalen Einheit zum Kinn: Mit 2 kräftigen Nähten (nicht resorbierbar) wird der Schildknorpel so am Hyoid fixiert, dass das Hyoid hinter dem Schildknorpelvorderrand zu liegen kommt [251]. Als Nahtmaterial werden Polytetrafluoroethylen (Goretex; permanent) und Vicryl 0 verwendet. Um ein Durchschneiden der Nähte am Schildknorpel zu vermeiden, erfolgt die Naht über Gore-tex-Sheets ( $\odot$ Abb. 26a).

Nach der Pexie werden die Fäden lang gelassen, um für die Suspension zur Verfügung zu stehen. Dazu wird ein submentaler Hautschnitt angelegt, das Gewebe zwischen Hyoid und Kinn auf der Muskulatur untertunnelt und vor der Austrittsstelle des 

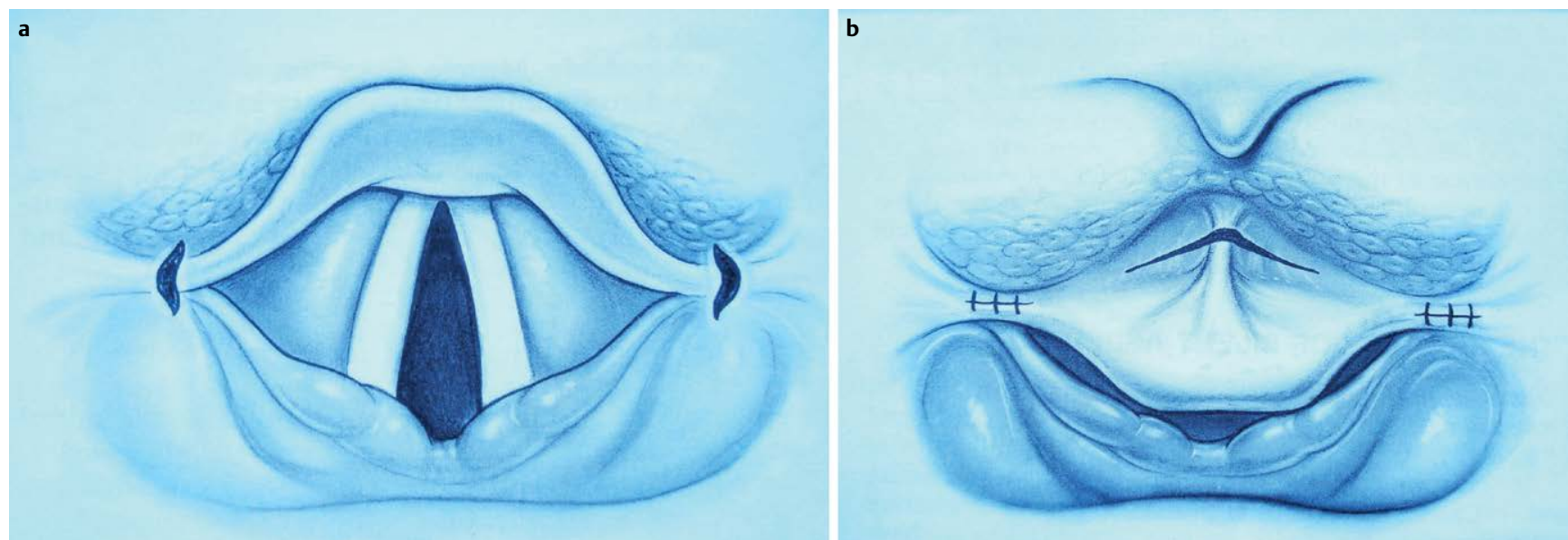

Abb. 27 a Zur Epiglottisabsenkung können die Plicae verlängert bzw. durchtrennt werden. b Nachdem die Plicae pharyngoepiglotticae quer durchtrennt und längs vernäht sind, ist zusätzlich schrittweises Lösen der Plica glossoepiglottica möglich.

N. mentalis 2 Bohrlöcher angelegt. Für die Suspension zum Kinn empfiehlt es sich, Polytetrafluoroethylen (Gore-tex; permanent) und 2 Ethibond Nähte zu verwenden. Alternativ kann auch die Sehne des M. plantaris longus, die neben der Achillessehne (ca. $30 \mathrm{~cm}$ lang) liegt, oder die des M. palmaris longus (ca. $12 \mathrm{~cm}$ lang) oder ein kräftiger Fascia lata Streifen als Patienten-eigenes Material gewählt werden. Über einen kleinen Hautschnitt $(2 \mathrm{~cm})$ neben dem Knöchel wird die Sehne aufgefunden und gestrippt. Das freie Transplantat verbindet Schildknorpel und Hyoid. Es wird zum Kinn weitergeführt, eingefädelt und mit einer fortlaufenden Sehnennahttechnik - dem Material angepasst - vernäht (० Abb. 26b, c) [247].

4. Um einer Überkorrektur, d.h. einem Verschluss des Larynxeingangs vorzubeugen, werden die Nähte für die Suspension selbst unter endoskopischer Kontrolle geknüpft.

5. Das Tracheostoma: da die Kehlkopf-Hyoid-Einheit nach cranial und ventral verlagert wird, muss am Ende das jetzt höher-liegende Tracheostoma neu angelegt werden.

Die PEG-Ernährung und eine geblockte Kanüle werden in der Regel für ca. 10 Tage beibehalten.

Die Langzeitergebnisse dieser Technik ( $n=17$; durchschnittlicher Beobachtungszeitraum 4,4 Jahre) werden mit 53\% ( $n=17)$ Erfolg d.h. ausschließlich orale Ernährung, mit 18\% Teilerfolg d.h. Langzeiternährung oral mit modifizierter Konsistenz und $30 \%$ Misserfolge d.h. keine oder sehr reduzierte, orale Ernährung angegeben [252].

5.4.1.8 Protektion des Larynxeingangs (Epiglottisabsenkung). Der Kehldeckel wird durch das thyroepiglottische Ligament, das präepiglottische Fettgewebe, die glossoepiglottische Falte und die beiden pharyngoepiglottische Falten in seiner leicht gebeugten Position gehalten.

Endoskopisch kann die Epiglottis zum Schutz des Larynxeingangs nach dorsal abgesenkt werden in einer Technik nach Laurian [253] oder, modifiziert, indem man die Plicae pharyngoepiglotticae bilateral durchtrennt und die Plica glossoepiglottica von der Zunge ablöst ( $\bullet$ Abb. 27a, b) [37].

5.4.1.9 Glottisschlussinsuffizienz und Dysphagie. Glottisschlussinsuffizienzen am membranösen oder/und knorpeligen Anteil der Glottis durch einseitige Lähmungen und Defekte sind permanent oder transitorisch, partiell oder total [254]. Sie können zu Penetration und Aspiration führen. Zur Verbesserung des
Schluckens werden verschiedene Therapieverfahren angewandt (modifiziert nach Giraldez-Rodriguez [255], ○ Abb. 28a - c, 29):

- Schlucktraining

- Injektions- und Implantationsaugmentation

- Thyroplastik \pm Arytenoid-Adduktion

- Partielle unilaterale Pharynx-Resektion

- Myotomie des M. cricopharyngeus

- Tracheotomie.

Injektions- und Implantationsaugmentation. Injektions- und Implantationsaugmentationen können transoral und transzervikal durchgeführt werden und kommen vorrangig bei der Behandlung von geringen bis mittelgradigen Glottisinsuffizienzen zum Einsatz ( $\bullet$ Abb. 28a, b). So werden diese Methoden zwar immer noch am häufigsten bei totalen und auch partiellen Stimmlippenlähmungen durchgeführt, jedoch werden sie auch zur Behandlung von Stimmlippenatrophien, bspw. beim Presbylarynx sowie bei Stimmlippennarben eingesetzt. Sie begleiten immer häufiger auch erfolgreich Verfahren der Larynxskelettchirurgie.

Ziel ist die Medialisierung der Stimmlippe durch einen Volumenaufbau (Augmentation) der insuffizienten Stimmlippe, z.B. bei Stimmlippenlähmungen, - defekten und -narben. Hierbei wird lateral in die Pars lateralis des M. vocalis bzw. tiefer zwischen Schildknorpel und M. vocalis injiziert bzw. implantiert. Als Material zur Augmentation werden unter anderem Fett, Faszie, Knorpel, Hyaluronsäure, Hydroxylapatit und Silikon verwendet [254].

Thyroplastik. Isshiki machte die Medialisierungsthyroplastik als Typ-I-Thyroplastik international bekannt [256]. Sie wird vorrangig bei Lähmungen und Atrophien der Stimmlippen aber auch bei Dysphagie eingesetzt. Die komplexe Struktur der für den Stimmklang relevanten Anteile der Stimmlippen wird nicht zerstört.

Nach vorheriger Anlage eines definierten Schildknorpelfensters wird eine Verlagerung der lateral gelegenen Stimmlippe zur Mitte hin durch das Einbringen und ggf. Verriegelung von körpereigenem oder körperfremdem Material erreicht. Dazu gehören bspw. körpereigener Knorpel (Septum- oder Schildknorpel) Gore-Tex, Titanspange oder Silikonblock [254] (๑ Abb. 28c, 29). 

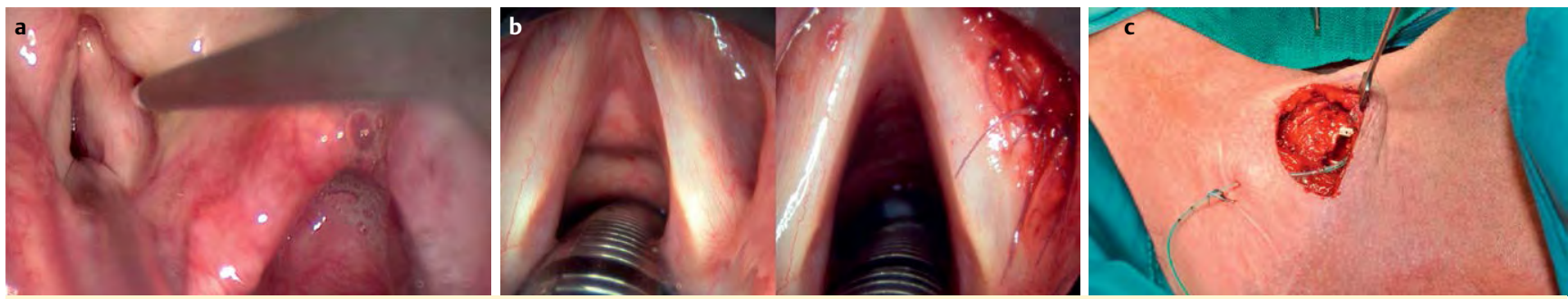

Abb. 28 a Injektionsaugmentation rechte Stimmlippe. b Implantationsaugmentation rechte Stimmlippe. c Linksseitige Thyroplastik Typ I mit Friedrichprothese.

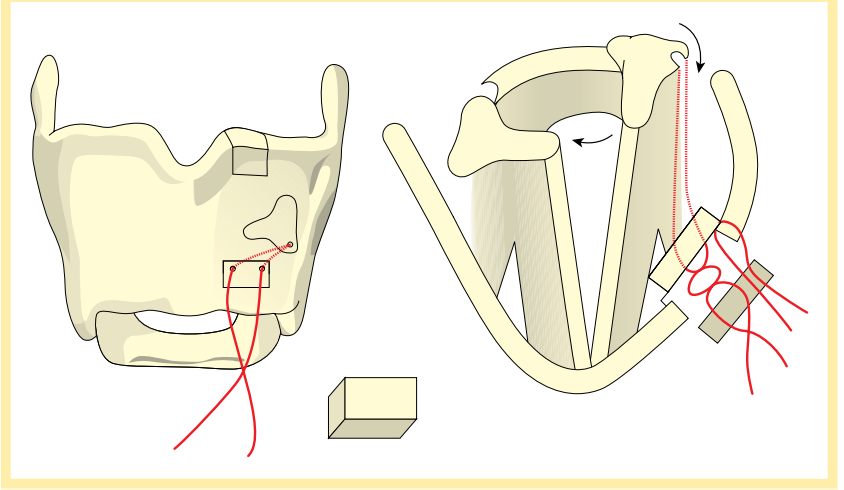

Abb. 29 Thyroplastik Typ I mit Arytenoid-Adduktion mit Knorpel.

Arytenoid-Adduktion. Die Arytenoid-Adduktion wurde erstmalig von Isshiki et al. 1978 als Operationstechnik zur Korrektur der Glottisinsuffizienz beschrieben [256]. Sie wird meist mit einer Thyroplastik kombiniert ( $\bullet$ Abb. 29). Im Rahmen dieses Eingriffs wird zunächst die Hinterkante des Schildknorpels der betroffenen Seite aufgesucht und nach Anlegen eines Knorpelfensters nach Maragos der Proc. muscularis des Aryknorpels exponiert [257]. Ein Vicrylfaden $(4 \times 0)$ wird anschließend durch den Proc. muscularis gezogen und ventral am Unterrand des Thyroplastik-Knorpelfensters verknotet. Durch die Rotation des Aryknorpels wird ein besserer Glottisschluss im hinteren Anteil der Stimmlippen ermöglicht und damit die Stimm- und die Schluckfunktion optimiert.

5.4.1.10 Myotomie des oberen Speiseröhrensphinkters. Eine Myotomie ist indiziert, wenn der obere Speiseröhrensphinkter (OÖS) während des Schluckaktes nicht oder ungenügend erschlafft $[258,259]$. Bei neurologischen Erkrankungen sollten die Entscheidung und der Zeitplan in Absprache mit dem interdisziplinären Team erfolgen. Vor dem Eingriff muss die Funktion des unteren Speiseröhrensphinkters (UÖS) kontrolliert und dokumentiert werden. Im Fall chronischer Refluxprobleme muss die Indikation zur Operation kritisch geprüft werden. Ggf. muss eine Behandlung des UÖS in das Konzept integriert werden. Ein Test mit Botulinumtoxin vor dem Eingriff kann hilfreich sein. In manchen Fällen kann Botulinum-Toxin als alleinige Methode ausreichen. Lokalisation und Ausmaß der Myotomie hängt vom Sitz und der Ausdehnung der muskulären Störung ab, die sich während der Videofluoroskopie im seitlichen Strahlengang darstellt.

Vor dem Eingriff wird eine Sengstaken-Blakemore Sonde in den oberen Ösophagus und den Hypopharynx gelegt. Sie wird mit 40 - $50 \mathrm{ml}$ Wasser bei Frauen und bei Männern mit $50-60 \mathrm{ml}$ Wasser gefüllt.
Die Schnittführung verläuft am Vorderrand des M. sternocleidomastoideus oder in einer Hautfalte in Höhe des Ringknorpels. Der prävertebrale Raum wird zwischen dem Gefäßnervenstrang von A. carotis, V. jugularis und N. vagus und dem Hypopharynx erreicht. Der äußere Ast des N. laryngeus superior muss erhalten bleiben.

Um nicht die nervöse Versorgung des Pharynx und Ösophagus zu gefährden, ziehen wir eine dorsomediale Myotomie vor. Der Hypopharynx wird mit der eingelegten Sengstaken-BlakemoreSonde zum Operateur gewendet. Die Myotomie der Konstriktormuskulatur folgt ( $\odot$ Abb. 26a, b).

Über dem venösen Plexus und der Schleimhaut unter der Muskulatur wird die Myotomie zum oberen Ösophagus und zum M. thyropharyngeus hin erweitert. Anhand des radiologischen Befundes wird die Ausdehnung der Myotomie bestimmt. Feinste Muskelfasern, die den Pharynx umschlingen, müssen durchtrennt werden, um Misserfolgen vorzubeugen. Diese Fasern können auf dem Cuff der Sengstaken-Blakemore-Sonde leicht identifiziert werden. Kleine Verletzungen der Mukosa werden durch atraumatische Naht verschlossen. In diesem Fall wird eine Nährsonde für wenige Tage gelegt. Die Möglichkeit, dass die Muskelstümpfe wieder miteinander verwachsen, wird dadurch ausgeschlossen, dass sie auf den Konstriktor oder den M. longus colli derselben Seite genäht werden [247].

Nachteil der lateralen Myotomien ist, dass die Nervenversorgung des Konstriktors auf der Seite der Myotomie teilweise zerstört wird.

Partielle unilaterale Pharynx-Resektion. In den seltenen Fällen einer Vagusparalyse kann die Ausweitung des Pharynx so exzessiv sein, dass eine partielle Pharynxresektion der gelähmten Seite durchgeführt werden muss. Die Resektionsgrenzen werden bei der videoendoskopischen Untersuchung vor dem Eingriff durch kleine Farbinjektionen markiert.

Das chirurgische Vorgehen bei der Pharynxteilresektion entspricht dem der Myotomie. Die Sengstaken-Blakemore-Sonde füllt den paralysierten Pharynx [247].

Die Muskelatrophie als Folge der Paralyse kann die Ursache für eine hauchdünne Pharynxwand sein. Gemeinsam mit den Farbinjektionen bestimmt die Wandstärke die Grenzen der Resektion. Wenn eine nasale Aspiration besteht, muss der gelähmte Teil des Nasopharynx verschlossen werden. Die Naht des Pharynx-Defektes ist identisch mit dem primären Verschluss nach unilateraler Pharynxtumorresektion. In der Regel sind diese Patienten bereits mit einer PEG versorgt. 2 Wochen nach der chirurgischen Versorgung beginnt das Schlucktraining.

In den Fällen, in denen eine Divertikel-Chirurgie oder eine partielle einseitige Pharynxresektion erfolgte, sollte gleichzeitig eine Myotomie bis in den Ösophagus durchgeführt werden. 

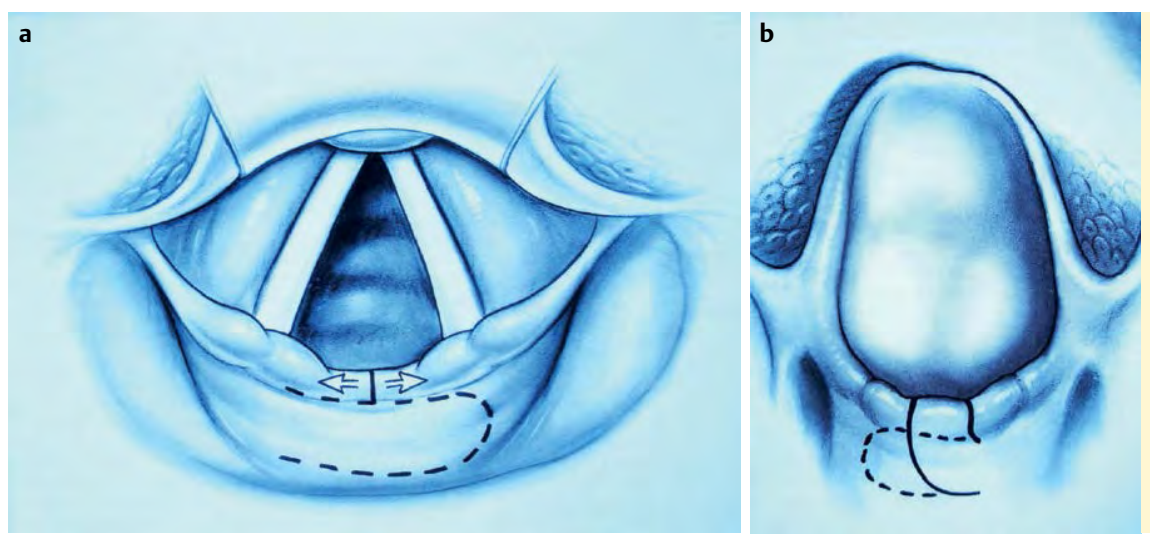

Abb. 30 a Eine niedrige Interarytenoidregion kann das Überlaufen von Speichel in den Larynx verursachen. Therapieoption durch kleinen Transpositionslappen. b Großvolumiger Transpositionslappen aus der Postkrikoidregion, um den Schutzwall an der Interarytenoidregion aufzubauen.
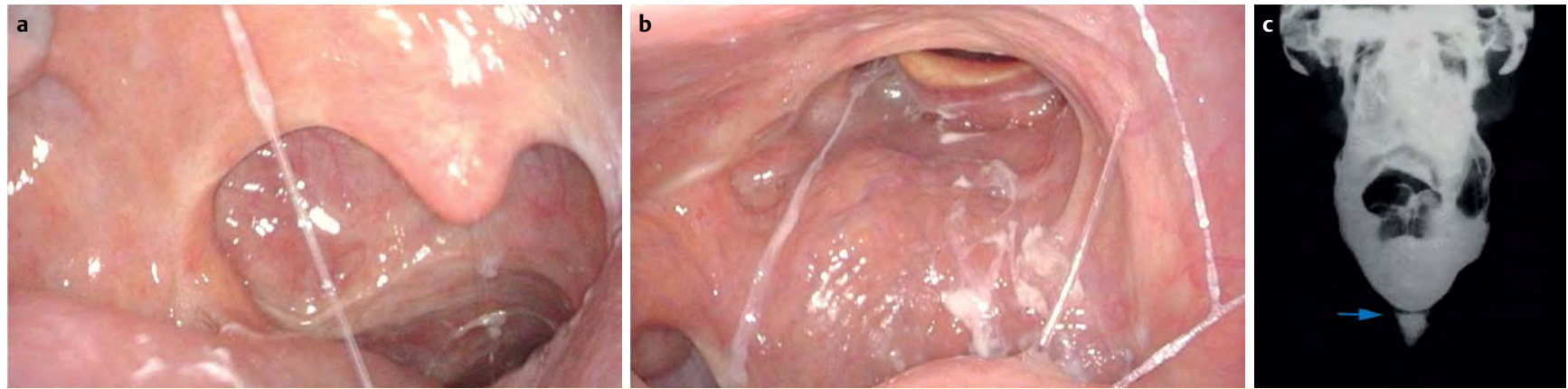

Abb. 31 a 87-jähriger Patient mit ausgeprägter oropharyngealer Dysphagie nach ausgedehnter Oropharynxteilresektion rechts und Neck dissection rechts und Radiochemotherapie (transorale Endoskopie). b Sehr visköser Speichel. Narbig verzogenes Gaumensegel rechts. Narbensegel ziehen über die Oropharynxhinterwand zur Gegenseite. Zusätzlich besteht eine Transportstörung der Zunge (transnasale Endoskopie). c Web- oder Segelbildungen (Pfeil) im Pharynx oder Ösophagus verursachen Globusgefühl und/oder dysphagische Beschwerden (Röntgenbild, anderer Patient).

5.4.1.11 Elevationsplastik der Interaryteniodregion. In den Fällen einer leichten Interarytenoid-Aspiration, bei denen keine oder eine geringe Progression der Erkrankung besteht, kann ein endoskopischer, plastisch-chirurgischer Eingriff das bestehende Problem vermindern. Speichel oder Flüssigkeiten laufen über den niedrigsten Punkt, der Interarytenoidregion in den Larynx. Ein postkrikoidaler Transpositionslappen, der auch bei kleinen Kehlkopfspalten (Grad 1) zur Anwendung kommt, kann eine Erhöhung der Schwelle (Interarytenoidregion) bewirken (o Abb. 30a, b).

Altersbedingte Schluckstörungen oder beginnende neurodegenerative Erkrankungen (M. Parkinson) zeigen vermehrt diese Form der Dysphagie. Patienten klagen über Räusperzwang und Husten, beides vorzugsweise beim Essen und Trinken. In den Fällen mit Sensibilitätsstörungen treten die Beschwerden auch in Ruhe auf. Die Indikation wird in interdisziplinärer Zusammenarbeit, nach der Funktionsendoskopie und der Videofluoroskopie gestellt. Eine krikopharyngeale Dysfunktion oder ein Tumor muss ausgeschlossen werden.

Nach der Tracheotomie wird der chirurgische Eingriff durchgeführt. Das Spreizlaryngoskop wird eingesetzt und die Interarytenoidregion dargestellt. Zur Behandlung der Aspiration wird ein Schleimhautlappen der postkrikoidalen Region umschnitten $[247,250]$ ( $\odot$ Abb. 30a). Nur der Schleimhautschnitt wird nach der Identifizierung der Arytenoidknorpel mit dem CO2-Laser ausgeführt. Die Schleimhaut der Interarytenoid-Region wird zur Seite präpariert. Der Lappen wird postkrikoidal freipräpariert. Er wird als Transpositionslappen reichlich dimensioniert und in das vorbereitete Bett der Interarytenoid-Region geschwenkt, mit Naht fixiert und an den Wundrändern mit Gewebekleber versiegelt ( $\bullet$ Abb. 30b).
5.4.1.12 Durchtrennung bei Segelbildung. Segel oder Webs kommen im Hypopharynx, oberen Ösophaguseingang und oberen Ösophagus als Entzündungsfolge vor. Diese narbigen Veränderungen lassen sich endoskopisch mit dem Laser durchtrennen und auflösen ( $\odot$ Abb. 31a-c).

\subsubsection{Spezielle Chirurgie bei besonders schwerer Aspiration}

Die stille Aspiration tritt häufig in Kombination mit motorischen Störungen auf und ist durch eine Sensibilitätsminderung gekennzeichnet. Wie bei den motorischen Ausfällen kann die Ausprägung der Störungen von sehr leichten bis zu den schweren Formen mit Sensibilitätsverlust reichen. Die Progredienz der Erkrankung sollte in die Planung des Therapiekonzeptes integriert werden. Um Zeit in derartigen Notfällen zu gewinnen und sich vom Problem der stillen Aspiration, die meist in Zusammenhang mit schweren neuromuskulären oder muskulären Störungen auftritt, zu befreien, müssen die Atemwege vor Aspiration geschützt werden.

Folgenden Befunde können während der Funktionsendoskopie erhoben werden:

- Überlauf in den Larynx ohne Husten

- Schaumspeichel im Oro- und Hypopharynx diffus verteilt, keine Entzündung

- Mangelnde Boluskontrolle

- Speisereste im Pharynx

- Eingedickter, zäher Klebespeichel bei gleichzeitig erhöhtem Reflux.

Wichtige Hinweise geben die anamnestischen Daten:

- TIA oder Schlaganfall

- Tumoren und/oder Folgen der Chirurgie in der hinteren Schädelgrube oder im Bulbusbereich

- Neurodegenerative Erkrankungen 
- Schädelhirntrauma

- Operationsfolgen - Narben, Defekte usw.

- Spätfolgen einer Radiochemotherapie usw.

- Frakturen, Strikturen und Stenosen usw..

Das Tracheostoma garantiert die Atmung. Nahrungszufuhr über die PEG sichert die Ernährung. Die stille Aspiration muss gestoppt werden. Kommunikation sollte möglich sein.

Wichtig ist die Klärung folgender Fragen:

- Ist die Atmung normal?

- Ist Stimmbildung möglich?

- Wie steht es mit der Muskelfunktion der Mundhöhle, des Pharynx, Larynx und der Trachea?

- Welche Innervation(en) ist/sind gestört?

-Wie ist das Allgemeinbefinden des Patienten?

- Wie ist die Prognose?

Von der Beantwortung dieser Fragen hängt die Entscheidung über eine chirurgische Intervention ab, die im interdisziplinären Team getroffen wird. Danach muss der Kopf-Hals-Chirurg die für diesen Patienten beste Behandlung finden und versuchen, diese an die individuelle Situation des Patienten zu adaptieren.

Wenn die Erkrankung des Patienten eine Phonation auf Glottisebene zulässt, sind diejenigen Techniken, die das ermöglichen, unbedingt vorzuziehen [247]. Wenn die für die Artikulation verantwortlichen Strukturen so geschädigt sind, dass die Patienten nicht mehr sprechen können, sind operative Verfahren diesbezüglich nicht angezeigt.

In bestimmten Fällen ist die Reversibilität dieser Chirurgie ein wichtiger Faktor. Aufgrund der Ergebnisse mit der reversiblen Technik dürfte die alte Technik der totalen Laryngektomie mit chirurgischer Stimmrehabilitation nur noch in sehr seltenen Fällen indiziert sein.

5.4.2.1 Laryngo-tracheale Separation. Die Indikation zur laryngo-trachealen Separation ist eine chirurgische Behandlung der stillen und/oder schweren Aspiration ohne Erhalt der individuellen Stimme, um eine komplette Trennung des Atem- und Schlucktraktes zu erzielen [260]. Sie ist für jeden Chirurgen eine ethisch schwere Entscheidung, da man chirurgisch normales laryngeales oder tracheales Gewebe alteriert und die Prozedur eine hohe Komplikationsrate von $58 \%$ und Ineffizienz in $27 \%$ hat [261]. Hara et al. [262] gaben dementsprechend eine erfolgreiche laryngo-tracheale Separation bei zwei Drittel ihrer neurologisch beeinträchtigten Kinder an. Die laryngo-tracheale Separation kann rückgängig gemacht werden.

5.4.2.2 Supraglottischer Verschluss des Kehlkopfes. Die supraglottische Separation kann transoral-endoskopisch vorgenommen werden. Hierbei wird die Epiglottis ventral und lateral mobilisiert und der Knorpel zur Reduktion der Spannung inzidiert sowie reduziert. Anschließend erfolgt die partielle Desepithelisierung der aryepiglottischen Falten. Der Epiglottislappen wird nun nach dorsal geschlagen und über dem Kehlkopfeingang mit den aryepiglottischen Falten vernäht. Die Phonation ist nach dem Verschluss nicht mehr möglich, aber er ist reversibel.

5.4.2.3 Plastischer Verschluss des Atemweges zwischen Larynx und Trachea mit Erhalt der individuellen Stimme und der Kontinuität des Paries membranaceus der Trachea. Ziel des reversiblen Trachealverschlusses mit Laryngo-Hyoidopexie (LHP), Suspension zum Kinn und nachfolgender Wiederherstellung der eigenen Stimme ist, dass der Patient/die Patientin nach dem Eingriff wieder mit der eigenen Stimme ohne Aspiration sprechen kann [259]. Die LHP mit Suspension ist notwendig, um die Quantität der Aspiration unterhalb der Stimmlippen und oberhalb des Verschlusses zu kontrollieren, da sonst die Stimmqualität beeinträchtigt werden kann.

Über eine mediane Schnittführung vom Schildknorpel bis in die Fossa jugularis wird die Trachea nach Durchtrennen des Schilddrüsenisthmus freigelegt. Die Trachea-Vorderwand wird in Höhe des ersten bis vierten Trachealringes in der Medianlinie gespalten oder in Form eines Türflügels geöffnet. Ein kleiner Tubus wird in Höhe des vierten Trachealringes zur Beatmung eingelegt.

Ein kranial gestielter Schleimhautlappen des Paries membranaceus wird umschnitten, nach oben geschlagen und so vernäht, dass er die Trachea in Höhe des ersten, ggf. zweiten Trachealringes mit der Schleimhaut nach oben spannungsfrei verschließt. Die kaudale Abdeckung des Verschlusses erfolgt mit einem Haut-Platysma-Faszien-Insellappen ( $\bullet$ Abb. 32a-d).

Nach der Planung des Lappens wird der schraffierte Bereich des Lappenstils desepithelisiert, sodass er unter der Haut einheilen kann. Der Lappenstiel sollte ausreichend groß gewählt werden, um eine gute Ernährung der Hautinsel sicherzustellen. Die Hautinsel wird so dimensioniert, dass sie sowohl die Unterseite des eingenähten, kranial gestielten Schleimhautlappen als auch den Entnahmedefekt an der Trachea-Rückwand spannungsfrei abdecken kann. Beide Lappen werden miteinander durch Fibrin verklebt. Um Druck auf den Lappenstiel zu vermeiden, wird die vordere Spange des zweiten Trachealrings reseziert. Eine leichte Tamponade stabilisiert vorübergehend die Position des Insellappens. Es empfiehlt sich ein etwas größeres Tracheostoma anzulegen und an der oberen Zirkumferenz des Tracheostomas die Halshaut mit dem Haut-Platysma-Faszien-Insellappen spannungsfrei zu vernähen. Dasselbe gilt für die Naht des übrigen Tracheostomas zwischen Halshaut und Trachealschleimhaut. Auf das Einlegen einer Kanüle kann nach der Extubation in der Regel verzichtet werden.

2 Monate nach diesem Eingriff folgt die sekundäre Wiederherstellung der Stimme durch eine Punktion durch den Trachealverschluss mit Einsatz einer Stimmprothese ( $\bullet$ Abb. 33a, b).

Das logopädische Training beginnt eine Woche nach dem Einsetzen des Shuntventils.

Auf diese Weise wurden bisher 3 Patienten erfolgreich versorgt. Einer dieser Patienten litt unter einem langsam wachsenden malignen Tumor. Die Patienten sprechen/sprachen mit ihrer eigenen Stimme. Die maximale Beobachtungszeit mit dieser Technik beträgt 22 Jahre. Die Patientin erblindete 1991 an einem Hämangioneuroblastom und verlor nach der Operation die Funktion der Hirnnerven IX, X, XI, XII. Die Folge war u.a. ein Myoklonus des Gaumensegels, Pharynx und Larynx, sowie ein vollständiger Sensibilitätsverlust von Pharynx, Larynx und Trachea. In der Zwischenzeit traten bei der Patientin 3 weitere Hämangioneuroblastome im Bereich der Medulla oblongata auf, die operativ entfernt wurden. Weitere Sensibilitätsverluste an den Händen und im Bereich des Halses waren die Folge, sodass die Patientin heute das Tracheostoma mit dem Handrücken verschließen muss. Sie findet das Tracheostoma mit dem Finger nicht mehr. Mit diesem Problem hat sie ein Universitätsstudium abgeschlossen und ist seit Jahren verheiratet.

In dem genannten, langen Beobachtungszeitraum, wie auch bei den anderen beiden Patienten traten als einzige Komplikation Granulationen am Shunt auf. Die Ventile müssen bei dieser Technik im Durchschnitt alle 7 - 10 Monate gewechselt werden. 

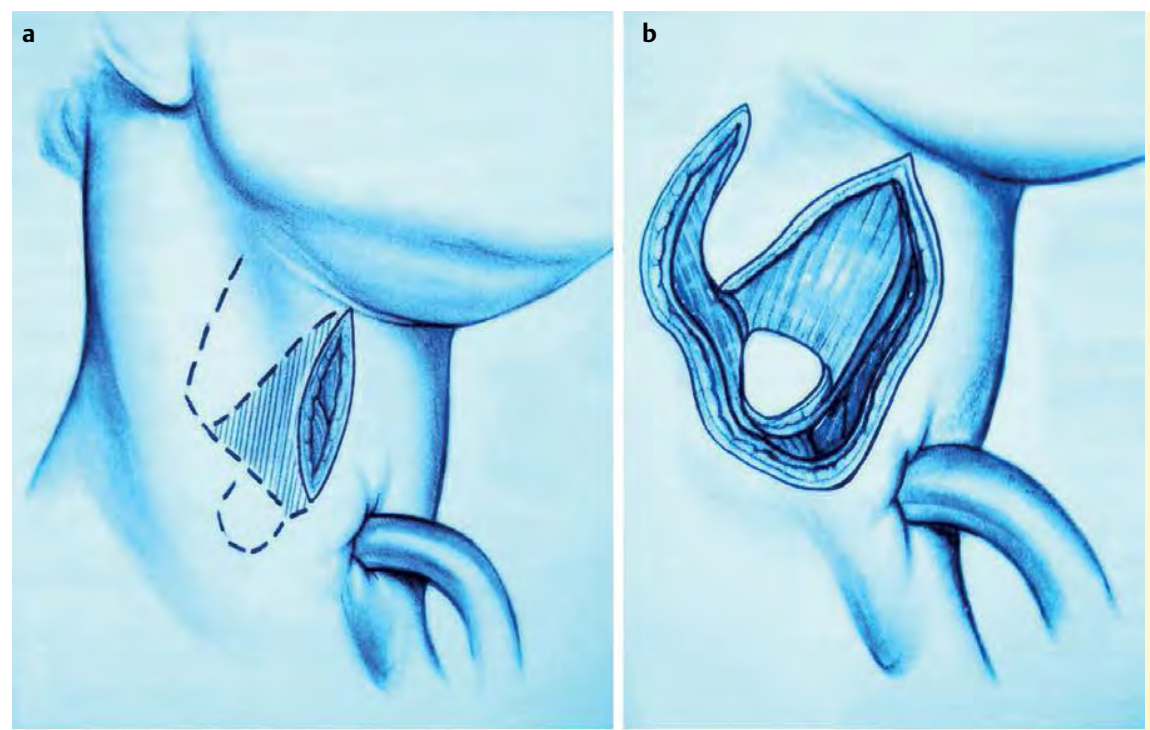

Abb. 32 a Schnittführung für den Haut-Platysma-Faszien-Insellappen. Schraffiert $=$ Teile des Lappens werden desepithelisiert, um sie unter die Haut zu legen. Über den Paramedianschnitt Darstellung von Trachea und Ringknorpel. b Die Hautinsel des Lappens ist umschnitten. Das Platysma mit seiner Faszie auf der Unterseite wird von der Halsmuskulatur präpariert. c Nach Einnähen des kranial gestielten Schleimhautlappens von der Trachearückwand als larynxseitige Bedeckung wird die Hautinsel zur tracheaseitigen Abdeckung als auch zur Defektdeckung der Entnahmestelle des Schleimhautlappens verwendet. $\mathbf{d}$ Entnahmestelle des kranial gestielten Schleimhautlappens der Tracheahinterwand (Stern) ist sichtbar. Teilentfernung einer Spange des 1. und/oder 2. Trachealrings, um Druck auf den Lappenstiel zu vermeiden.
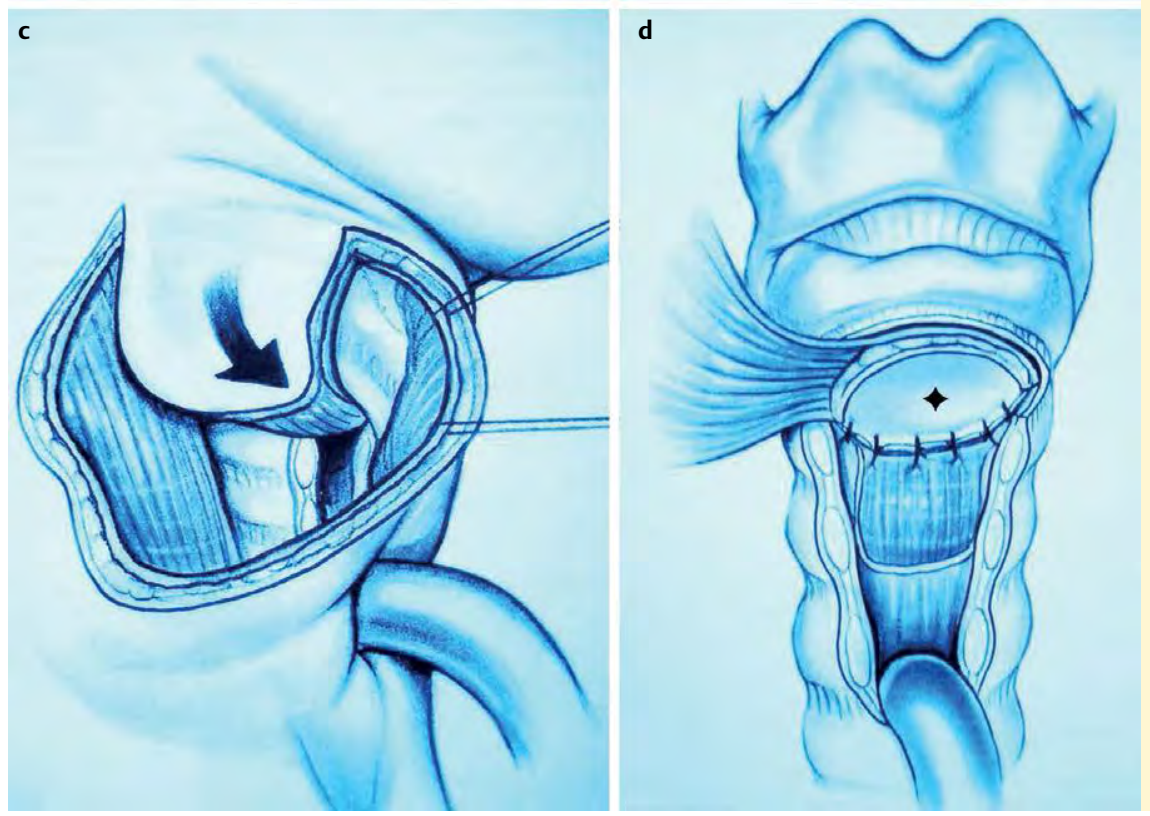

Weitere Techniken wurden beschrieben, bei denen die Ultima Ratio der Laryngektomie vermieden werden kann, und die Phonation über eine Stimmprothese im Pharynx möglich ist [263].

5.4.2.4 Laryngektomie. Dysphagie und Laryngektomie können auf verschiedene Weise miteinander zusammenhängen. Die laryngotracheale Separation oder Laryngektomie werden bei irreversiblen, neurologischen oder onkologischen Erkrankungen mit schwerer Aspiration therapeutisch eingesetzt. Die Laryngektomie ist die radikalste Methode zur Prävention der Aspiration. Laryngektomien mit Hypopharynxteilresektionen führen zu Schluckstörungen ( $\bullet$ Abb. 11).

Eine schwere Dysphagie ist nicht selten das Ergebnis der Therapie fortgeschrittener Larynx- und Hypopharynxkarzinome. Hierbei weisen Patienten nach einer Radiochemotherapie und/ oder Laryngektomie die schlechtesten Ergebnisse der Schluckfunktion auf [264]. Einer Stenose im Neopharynx kann man durch die primäre Verwendung eines Radialislappens vorbeugen. Die Bougierung der Stenose verbessert unter Umständen die Situation für den Patienten. Sweeny et al. [265] werteten Stenosen des Neopharynx bzw. des pharyngoösophagealen Übergangs nach Laryngektomie aus. Hierbei traten Stenosen in
19\% der laryngektomierten Patienten auf. 82\% dieser Stenosen fielen im ersten postoperativen Jahr auf. Es gab keinen Unterschied zwischen primär oder Salvage-Laryngektomierten. Patienten, die nur einmal bougiert bzw. dilatiert werden mussten, hatten eine bessere Prognose bzgl. der Dysphagie im Vergleich zu Laryngektomierten mit mehrfachen Dilatationen.

\subsubsection{Chirurgische Therapie von Schluckstörungen nach Radiochemotherapie}

Nguyen [39] konnte bei Patienten mit Kopf-Hals-Karzinomen nach primärer Radiochemotherapie oder chirurgischer Therapie in Kombination mit einer Radiochemotherapie in 11\% Stenosen im Pharynx oder Ösophagus mittels modifizierter fluoroskopischer Barium-Schluckuntersuchung und Endoskopie nachweisen, die posttherapeutisch zu persistierender Dysphagie führten. Diese Stenosen wurden durch Dilatation behandelt. In 50\% war eine einmalige Dilatation zur Dysphagietherapie ausreichend. In $21 \%$ wurde mindestens $4 \times$ dilatiert.

Hutcheson et al. [266] analysierten 23 rezidivfreie Patienten mit laryngopharyngealer Dysfunktion, die aufgrund ihrer Tumorbehandlung laryngektomiert werden mussten ( $\bullet$ Tab. 23). Dies waren $6 \%$ aller totalen Laryngektomien in einem untersuchten 

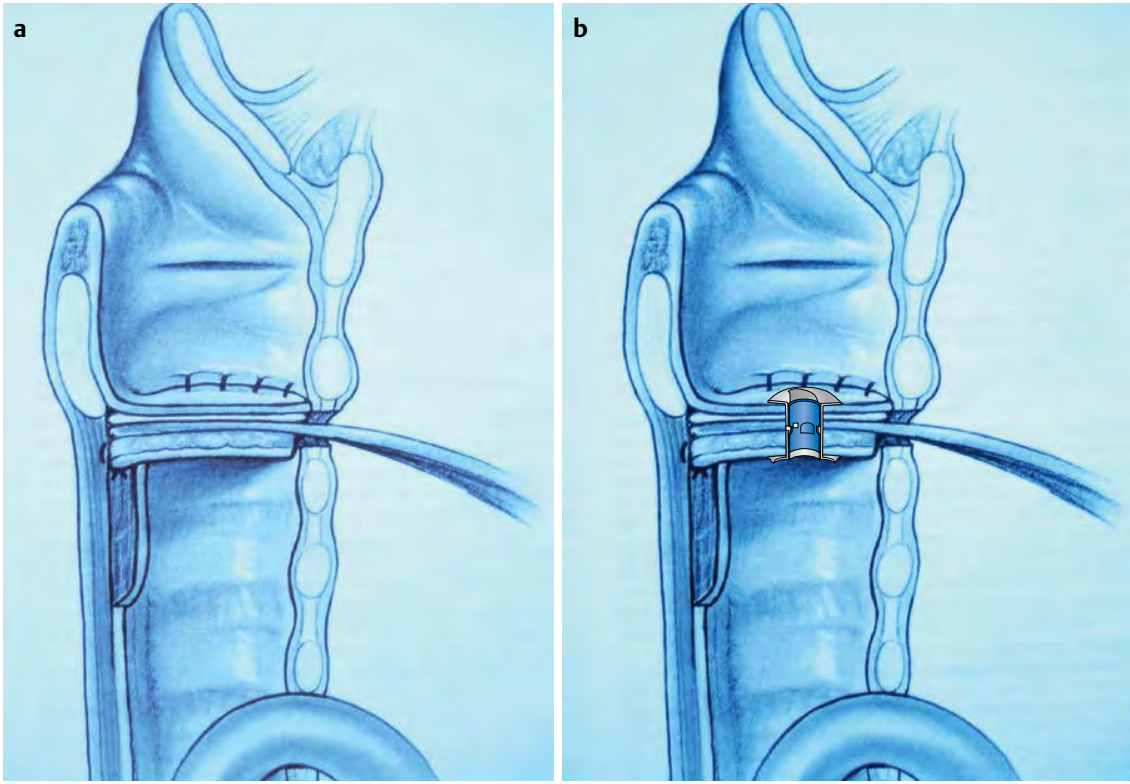

Abb. 33 a Die Entnahmestelle des kranial gestielten Schleimhautlappens wird durch den Haut-Platysma-Faszie-Insellappen gedeckt. b 2 Monate nach Abheilen wird ein Shuntventil nach Punktion in den Trachea-Verschluss eingesetzt, Sprechen ist mit der eigenen, individuellen Stimme möglich.
Zeitraum von 6 Jahren. Alle Patienten mit laryngopharyngealer Dysfunktion hatten sich aufgrund eines Karzinoms im KopfHals-Bereich primär einer Strahlentherapie oder Radiochemotherapie unterzogen.

\subsubsection{Tracheotomie}

Zahlreiche Patienten auf Intensivstationen benötigen zur Aufrechterhaltung der Atmung einen Zugang zu den tiefen Atemwegen. Indikationen sind prolongierte Intubation, Unterstützung der Beatmung, pulmonales Sekretmanagement, Obstruktion der oberen Atemwege mit Stridor, Atemnot, interkostalen Einziehungen, OSAS, beidseitige Stimmlippenlähmung, fehlende Möglichkeit der Intubation, große Eingriffe in der Kopf-Hals-Region, Traumamanagement, Schutz der Atemwege bei neurologischen Erkrankungen [267].

Der Atemweg wird durch einen geblockten Tubus in Sedierung als Kurzzeitmaßnahme abgedichtet.

Eine frühe Tracheotomie, etwa 72 Stunden nach Intubation bringt keine Nachteile [268]. Grundsätzlich ist sie indiziert, wenn die Beatmung voraussichtlich länger als 12 Tage erforderlich ist [269].

Die Dysphagie mit Aspiration ist ein Grund, beim nicht-intubierten Patienten ein Tracheostoma anzulegen. Andererseits kann die Tracheotomie als Komplikation zu Dysphagie führen. Sie wird in der Regel entweder als perkutane Dilatations- oder als chirurgische Tracheotomie durchgeführt [270], wobei auf Intensivstationen die Dilatationstracheotomie häufiger eingesetzt wird und die gleiche Sicherheit besitzt [271,272].

Kontraindikationen gegen die Punktionstracheotomie nach Bause [273] sind eine akute Ateminsuffizienz (Notfallsituation), nicht korrigierbare kombinierte Gerinnungsstörungen sowie die hochgradige Kreislaufinstabilität.

Indikationen für die konventionelle Tracheotomie/Tracheostomie:

- Patienten unter 18 Jahren

- Unmöglichkeit der Trachealpunktion

- Instabile Halswirbelsäulenfrakturen

- Trachealtumoren

- Alle supratrachealen Atemwegshindernisse

- Frische Trachealnaht

- Notwendige Kanülengröße > $10 \mathrm{~mm}$ (Innendurchmesser)

- Notwendigkeit der seitengetrennten Beatmung
Tab. 23 Indikationen zur totalen Laryngektomie bei Patienten mit laryngopharyngealer Dysfunktion nach Hutcheson [266] - Komplikationen und funktionelle Störungen:

\begin{tabular}{|c|c|}
\hline \multicolumn{2}{|l|}{ Dysphagie } \\
\hline Aspiration & $95,7 \%$ \\
\hline Strikturen & $30,4 \%$ \\
\hline Enterale/parenterale Ernährung & $73,9 \%$ \\
\hline Nihil per os & $56,5 \%$ \\
\hline Osteo-/Chondroradionekrose & $26,1 \%$ \\
\hline Atemwege/Tracheotomie & $39,1 \%$ \\
\hline \multicolumn{2}{|l|}{ Pneumonien } \\
\hline keine & $30,4 \%$ \\
\hline einmalige Episode & $17,4 \%$ \\
\hline rezidivierend/chronisch & $52,2 \%$ \\
\hline
\end{tabular}

- Frische Bronchusnaht

- Vorbestehende Tracheomalazie

- Endgültiges Stroma

- Mobile, nicht-beatmete Patienten

Für die offene chirurgische Tracheotomie werden die Durchtrennung oder Verdrängung des Isthmus der Schilddrüse nach kaudal, die Inzision zwischen dem 2. und 3. Trachealring empfohlen [270,274]. Ein Björk-Lappen ist nicht erforderlich. Bei erwarteten Langzeit-Tracheotomien ist eine sorgfältige Haut-Schleimhaut-Naht für ein stabiles Tracheostoma wünschenswert, um Granulationen und Stenosen zu vermeiden und die Beatmung zu sichern (Tracheostomie).

Operationsmethode: ca. $4 \mathrm{~cm}$ langer quer verlaufender Hautschnitt. Die Linea alba wird dargestellt, die infrahyoidale Muskulatur wird nach lateral und der Schilddrüsenisthmus nach kaudal verdrängt bzw. durchtrennt. Die Trachea wird meist zwischen dem 2. und 3. Trachealring eröffnet. Die 2. Trachealspange wird subkutan mit dem oberen und die 3. subkutan mit dem unteren Hautschnittrand vernäht. Intensiv- und Langzeit-Beatmungspatienten können dagegen in gleicher Weise bei tief sitzendem Larynx ein zwischen dem 1. und 2. Trachealring gelegenes dauerhaftes Stoma bekommen. Sonst erweitert sich das Tracheostoma durch das druckbedingte Aufwärtswandern der Kanüle. Dies erschwert das Abdichten der Kanüle bei beatmeten 
Patienten. Die letztgenannte Technik hat folgende Vorteile in Bezug auf Schluckstörungen: sichere Öffnung durch Adaptation der Stomaränder an den Hautschnitt und kleineres Tracheostoma. Eine Variante der Trachealeröffnung ist die senkrechte Inzision über mehrere Trachealringe [267]. Dieses Vorgehen empfiehlt sich in Notfällen wegen der besseren Übersicht.

Das Wechseln der Kanüle ist ab dem 2. postoperativen Tag möglich. Ein Sprechventil, auf die Trachealkanüle aufgesetzt, fördert den Erfolg der Stimm- und Schlucktherapie [275].

Eine geblockte Trachealkanüle reduziert das Hauptrisiko der Dysphagie, die Aspiration. Sie kann aber das Überlaufen von Speichel in die Luftröhre nicht verhindern. Eine transnasal gelegte Nährsonde erhöht die Speichelproduktion. Da die normale Speichelproduktion mit ca. 0,6-1,5l pro Tag zu viel für den Atemweg ist - auch wenn nur ein Teil aspiriert wird - muss der Patient sorgfältig überwacht werden. Der Speichel staut sich bei Aspiration über dem Cuff und kann über die subglottische Absaugung der geblockten Trachealkanüle abgesaugt werden. Eine professionelle Trachea-Pflege ist notwendig, um Entzündungen der trachealen Punktionswunde bedingt durch Speichel und Keimbesiedlung zu vermeiden.

\subsubsection{Perkutane endoskopische Gastrostomie (PEG)}

Sie ist ein etabliertes endoskopisch chirurgisches Verfahren für Patienten mit Dysphagie, die sich über eine absehbare Zeit oder auch dauerhaft nicht die notwendige tägliche Flüssigkeits- und Kalorienmenge zuführen können. Hierbei sichert die Nahrungszufuhr über die PEG-Sonde den Energiebedarf. Indikationen für eine PEG-Anlage sind obstruktive oder neurogene Dysphagien mit reversiblen und irreversiblen Schluckstörungen sowie Aspiration [276]. Die Patienten können sich im Rahmen ihrer Möglichkeiten weiter oral ernähren. Derzeit haben sich die Fadendurchzugmethode in der Seldinger-Technik und die Direktpunktion als Verfahren etabliert, wobei die Fadendurchzugmethode als sichere und einfache Methode am häufigsten angewandt wird. In prospektiven klinischen Studien konnte nachgewiesen werden, dass die Ernährung über die PEG-Sonden sehr gut akzeptiert und toleriert wird. Mays et al. [277] entwickelten Prädiktoren für die Anlage von Magensonden, um die enterale Ernährung im Rahmen der Therapie zu gewährleisten. Einflussfaktoren sind die präoperative Bestrahlung, supraglottische Larynxteilresektion, Tracheotomie, klinischer Halslymphknotenstatus (N0 vs. N2, N1 vs. N2), präoperativer Gewichtsverlust, Dysphagie, Art der Rekonstruktion und das Tumorstadium. Mays befürwortet die frühe Anlage der PEG, damit bei HochrisikoPatienten Komplikationen im Rahmen der postoperativen Wundheilung vermieden werden können und die Prognose sowie die Lebensqualität verbessert werden.

\section{Abstract}

\section{Position Paper of the German Society of Oto-Rhino- Laryngology, Head and Neck Surgery and the German Society of Phoniatrics and Pediatric Audiology - Current State of Clinical and Endoscopic Diagnostics, Evaluation, and Therapy of Swallowing Disorders in Children and Adults}

Swallowing disorders are frequent. The main concern is mortality due to aspiration induced pneumonia and malnutrition. On the other hand quality of life is severely affected. The demogra- phic trend indicates an increase of dysphagia in the future. Neurodegenerative diseases, tumors of the digestive tract and sequelae of tumor treatment in the head and neck region are the main pathologic entities.

Predominantly ENT physicians and phoniatrists, are asked for diagnostics and therapy who will coordinate the interdisciplinary treatment according to the endoscopic findings.

A differentiated approach in history, diagnostics, and symptom oriented treatment is necessary for the mostly complex disorders. The integration of non-medical personnel such as logopeds (speech language pathologists), physiotherapists, and occupational therapists in planning and executing an effective therapy expands and completes the patient-oriented care. Conservative treatment by these therapists is an important pillar in the treatment. Parts of the specific diagnostics can be taken over by them in close cooperation.

In particular an interdisciplinary cooperation with the staff from intensive care medicine is indispensable.

The diagnostic procedures of specific endoscopy as described in this position paper are part of the primary and fundamental tasks of ENT specialists and phoniatrists.

Endoscopy is a medical service that is basically not delegable. Consequently substitution of the physician is precluded.

\section{Literatur}

1 Remington T, Fagan SC. Drug-induced communication and swallowing disorders. In: Johnson A, Jacobson B (eds.). Medical Speech-Language-pathology. A practitioner's guide. New York: Thieme, 2007; 363-377

2 Langmore SE, Schatz K, Olsen N. Fiberoptic endoscopic examination of swallowing safety: a new procedure. Dysphagia 1988; 2: 216-219

3 Bader CA, Schütze H, Schick B. Zur Ausführung der fiberoptisch-endoskopischen Schluckdiagnostik durch nichtärztliche Therapeuten sowie durch Ärzte ohne HNO-ärztliche bzw. ohne phoniatrisch-pädaudiologische Gebietsbezeichnung: Ärztliche und juristische Aspekte. HNO 2013; 61: 970-974

4 Bader CA, Niemann G. Dysphagie bei Kindern mit Infantiler Zerebralparese - Fiberoptisch-endoskopische Befunde. Laryngorhinootologie 2010; 89: 90-94

5 Herrmann I. Transnasal Endoscopy. In: Johnston N, Toohill RJ (eds.). Effects, Diagnosis and Management of Extra-Esophageal Reflux: Nova Science Pub Inc, 2010

6 Herrmann IF, Scarpignato C. Funktionelle Endoskopie: Physiologische und pathophysiologische Grundlagen der Refluxerkrankung, ihre Diagnostik und Therapie. HNO 2009; 57: 1221-1236

7 Jungheim M, Miller S, Kuhn D, Schwemmle C, Schneider JP, Ochs M, Ptok M. Anatomie des oberen Osophagussphinkters. HNO 2014; 62: 385-392 quiz 393-404

8 Jungheim M, Miller S, Kuhn D, Schwemmle C, Schneider JP, Ochs $M$, Ptok M. Physiologie des oberen Osophagussphinkters. HNO 2014; 62 : 457-466 quiz 467-478

9 Herrmann IF, Arce-Recio S, Cirillo F, Bechi P. The role of functional endoscopy in understanding upper GI functioning. Acta Endoscopica 2008; 247-261

10 Doty RW, Bosma JF. An electromyographic analysis of reflex deglutition. J Neurophysiol 1956; 19: 44-60

11 Humbert IA, Robbins J. Normal swallowing and functional magnetic resonance imaging: a systematic review. Dysphagia 2007; 22: 266 275

12 Hamdy S, Rothwell JC, Brooks DJ, Bailey D, Aziz Q, Thompson DG. Identification of the cerebral loci processing human swallowing with H2(15)O PET activation. J Neurophysiol 1999; 81: 1917-1926

13 Daniels S, McAdam C, Brailey K, Foundas A. Clinical assessment of swallowing and prediction of dysphagia severity. Am J Speech Lang Pathol 1997; 6: 17-24

14 Teismann IK, Dziewas R, Steinstraeter O, Pantev C. Time-dependent hemispheric shift of the cortical control of volitional swallowing. Hum Brain Mapp 2009; 30: 92-100

15 Kim Y, McCullough GH. Stage transition duration in patients poststroke. Dysphagia 2007; 22: 299-305 
16 Prosiegel M, Weber S. Dysphagie: Diagnostik und Therapie: Ein Wegweiser für kompetentes Handeln. Berlin: Springer, 2013

17 Schröter-Morasch H, Graf S. Dysphagiediagnostik durch den HNOArzt. HNO 2014; 62: 324-334

18 Ney DM, Weiss JM, Kind AJ, Robbins J. Senescent swallowing: impact, strategies, and interventions. Nutr Clin Pract 2009; 24: 395-413

19 Jungheim $M$, Schwemmle C, Miller S, Kühn D, Ptok M. Schlucken und Schluckstörungen im Alter. HNO 2014; 62: 644-651

20 Garon BR, Huang Z, Hommeyer M, Eckmann D, Stern GA, Ormiston C. Epiglottic dysfunction: abnormal epiglottic movement patterns. Dysphagia 2002; 17: 57-68

21 Powell J, Cocks HC. Mucosal changes in laryngopharyngeal reflux prevalence, sensitivity, specificity and assessment. Laryngoscope 2013; 123: 985-991

22 Mesallam TA, Stemple JC, Sobeih TM, Elluru RG. Reflux symptom index versus reflux finding score. Ann Otol Rhinol Laryngol 2007; 116: 436-440

23 Leder SB, Suiter DM, Duffey D, Judson BL. Vocal fold immobility and aspiration status: a direct replication study. Dysphagia 2012; 27: 265-270

24 Schröter-Morasch H, Hoole P. Differenzialdiagnose hyperkinetischer Bewegungsstörungen des Kehlkopfs. In: Gross M, Eysholdt U (eds.). Aktuelle phoniatrisch-pädaudiologische Aspekte. Heidelberg: Median Verlag, 1998

25 Ota K, Saitoh E, Baba M, Sonoda S. The secretion severity rating scale: a potentially useful tool for management of acute-phase fasting stroke patients. J Stroke Cerebrovasc Dis 2011; 20: 183-187

26 Rodrigues B, Nobrega AC, Sampaio M, Argolo N, Melo A. Silent saliva aspiration in Parkinson's disease. Mov Disord 2011; 26: 138-141

27 Murray J, Langmore SE, Ginsberg S, Dostie A. The significance of accumulated oropharyngeal secretions and swallowing frequency in predicting aspiration. Dysphagia 1996; 11: 99-103

28 Abe $H$, Tsubahara $A$. Observation of arytenoid movement during laryngeal elevation using videoendoscopic evaluation of swallowing. Dysphagia $2011 ; 26$ : 150-154

29 Langmore SE. Endoscopic evaluation and treatment of swallowing disorders. New York: Thieme New York, 2011

30 Butler SG, Stuart A, Kemp S. Flexible endoscopic evaluation of swallowing in healthy young and older adults. Ann Otol Rhinol Laryngol 2009; 118: 99-106

31 Schröter-Morasch H, Ziegler W. Transorale Endoskopie des Schluckvermögens (TOES) - Entwicklung und Evaluation eines Untersuchungsbogens. In: Gross ME, Kruse E (eds.). Aktuelle phoniatrisch-pädaudiologische Aspekte. 2002/2003; 2002: 405-410

32 Sulcia L, Verheyden J, Blitzer A. The larynx and pharynx. Cricopharyngeal muscle spasm. In: Moore P, Naumann M (eds.). Handbook of botulinum toxin treatment. Malden, USA: Blackwell Science, 2003; 153-154

33 Achem SR, Devault KR. Dysphagia in aging. J Clin Gastroenterol 2005; 39: 357-371

34 Schindler JS, Kelly JH. Swallowing disorders in the elderly. Laryngoscope 2002; 112: 589-602

35 Stegemann S, Gosch M, Breitkreutz J. Swallowing dysfunction and dysphagia is an unrecognized challenge for oral drug therapy. Int J Pharm 2012; 430: 197-206

36 Herrmann IF. Surgical solutions for aspiration problems. J Jpn Bronchoesophagol Soc 1992; 43: 72-79

37 Herrmann IF, Arce-Recio S. Special techniques for resolving aspiration problems. Operative Techniques in Otolaryngology - Head and Neck Surgery 1999; 10: 244-252

38 Rosenthal DI, Lewin JS, Eisbruch A. Prevention and treatment of dysphagia and aspiration after chemoradiation for head and neck cancer. J Clin Oncol 2006; 24: 2636-2643

39 Nguyen NP, Smith HJ, Moltz CC, Frank C, Millar C, Dutta S, Sallah S. Prevalence of pharyngeal and esophageal stenosis following radiation for head and neck cancer. J Otolaryngol Head Neck Surg 2008; 37: 219-224

40 Frowen J, Cotton S, Corry J, Perry A. Impact of demographics, tumor characteristics, and treatment factors on swallowing after (chemo)radiotherapy for head and neck cancer. Head Neck 2010; 32: 513-528

41 Frowen J, Drosdowsky A, Perry A, Corry J. Long-term swallowing after chemoradiotherapy: A prospective study of functional and patientreported changes over time. Head Neck 2014

42 van den Berg MG, Rutten H, Rasmussen-Conrad EL, Knuijt S, Takes RP, van Herpen CM, Merkx MA. Nutritional status, food intake, and dysphagia in long-term survivors with head and neck cancer treated with chemoradiotherapy: a cross-sectional study. Head Neck 2014; 36: 60-65

43 Gourin CG, Starmer HM, Herbert RJ, Frick KD, Forastiere AA, Eisele DW, Quon $H$. Short- and long-term outcomes of laryngeal cancer care in the elderly. Laryngoscope 2014
44 Zenner HP. Die Schleuderverletzung der Halswirbelsäule und ihre Begutachtung. Berlin: Springer, 1987

45 Hülse $M$. Die funktionelle Dysphonie nach Halswirbeltrauma. Laryngorhinootologie 1991; 70: 599-603

46 Seifert K. Oto-Rhino-Laryngologie in Klinik und Praxis. Band III. Hals. Stuttgart, New York: Georg Thieme Verlag, 1995; 256-270

47 Bartholome G, Schröter-Morasch H. Schluckstörungen: Diagnostik und Rehabilitation. München: Elsevier, 2014

48 Walther EK. Vertebragene Dysphagie bei diffuser idiopathischer Skeletthyperostose (Morbus Forestier). Laryngorhinootologie 1991; 70: 604-608

49 Strasser G, Schima W, Schober E, Pokieser P, Kaider A, Denk DM. Cervical osteophytes impinging on the pharynx: importance of size and concurrent disorders for development of aspiration. AJR Am J Roentgenol 2000; 174: 449-453

50 Seidler TO, Perez Alvarez JC, Wonneberger K, Hacki T. Dysphagia caused by ventral osteophytes of the cervical spine: clinical and radiographic findings. Eur Arch Otorhinolaryngol 2009; 266: 285-291

51 Fountas KN, Kapsalaki EZ, Nikolakakos LG, Smisson HF, Johnston KW Grigorian AA, Robinson JS Jr. Anterior cervical discectomy and fusion associated complications. Spine (Phila Pa 1976) 2007; 32: 2310-2317

52 Mukherjee R, Muller M, Amstad H, Fournier J, Haile SR, Stockli SJ, Litschel $R$. Dysphonie und Dysphagie nach anterioren Zugängen zur Halswirbelsäule. HNO 2014; 62: 575-581

53 Daniels AH, Riew KD, Yoo JU, Ching A, Birchard KR, Kranenburg AJ, Hart $R A$. Adverse events associated with anterior cervical spine surgery. J Am Acad Orthop Surg 2008; 16: 729-738

54 Riley $L H 3^{\text {rd }}$, Vaccaro AR, Dettori JR, Hashimoto $R$. Postoperative dysphagia in anterior cervical spine surgery. Spine (Phila Pa 1976) 2010; 35: S76-S85

55 Bazaz R, Lee MJ, Yoo JU. Incidence of dysphagia after anterior cervical spine surgery: a prospective study. Spine. Spine (Phila Pa 1976) 2002; 27: 2453-2458

56 Kalb S, Reis MT, Cowperthwaite MC, Fox DJ, Lefevre R, Theodore N Sonntag VK. Dysphagia after anterior cervical spine surgery: incidence and risk factors. World Neurosurg 2012; 77: 183-187

57 Dziewas $R$, Glahn J, Helfer C, Ickenstein G, Keller J, Lapa S, Busse 0. FEES für neurogene Dysphagien: Ausbildungscurriculum der Deutschen Gesellschaft für Neurologie und Deutschen SchlaganfallGesellschaft. Nervenarzt 2014; 85: 1006-1015

58 Kikendall JW. Pill esophagitis. J Clin Gastroenterol 1999; 28: 298-305

59 Stoschus B, Allescher HD. Drug-induced dysphagia. Dysphagia 1993; 8: $154-159$

60 Forster A, Samaras $N$, Gold $G$, Samaras $D$. Oropharyngeal dysphagia in older adults: A review. European Geriatric Medicine 2011; 2: 356-362

61 Winkler-Budenhofer UC, Ihrler S, Göke B, Kolligs FT. Akute Exazerbation einer chronischen Ösophagitis. Dtsch Med Wochenschr 2009; 134: 1517-1519

62 Spieker MR. Evaluating dysphagia. Am Fam Physician 2000; 61: 36393648

63 Guttman OR, Zachos M. Drug-induced esophageal injury with an occult vascular ring. Paediatr Child Health 2011; 16: 554-556

64 Kato S, Komatsu K, Harada Y. Medication-induced esophagitis in children. Gastroenterol Jpn 1990; 25: 485-488

65 O'Sullivan T, Fagen SC. Drug-induced communication and swallowing disorders In: Johnson AF, Jacobson BH (eds.). Medical Speech-Language-pathology. A practitioner's guide. New York: Thieme, 1998; 363-377

66 Lim HC, Nigro MA, Beierwaltes $P$, Tolia V, Wishnow $R$. Nitrazepam-induced cricopharyngeal dysphagia, abnormal esophageal peristalsis and associated bronchospasm: probable cause of nitrazepam-related sudden death. Brain Dev 1992; 14: 309-314

67 Jung KW, Myung SJ, Jung HY. A patient with Dysphagia associated with opioid medication. J Neurogastroenterol Motil 2012; 18: 220 221

68 O'Neill JL, Remington TL. Drug-induced esophageal injuries and dysphagia. Ann Pharmacother 2003; 37: 1675-1684

69 Arai T, Sekizawa K, Ohrui T, Fujiwara H, Yoshimi N, Matsuoka H, Sasaki $H$. ACE inhibitors and protection against pneumonia in elderly patients with stroke. Neurology 2005; 64: 573-574

70 Sokoloff LG, Pavlakovic R. Neuroleptic-induced dysphagia. Dysphagia 1997; 12: 177-179

71 Finsterer J. Medikamenteninduzierte Myopathien. Nervenarzt 2006; 77: 682-686, 688-693

72 Schoser BG, Pongratz D. Lipidsenker- und andere toxische Myopathien. Internist (Berl) 2005; 46: 1198-1206 
73 Schwemmle C, Ptok M. Die Behandlung laryngealer Bewegungsstörungen mit Botulinumtoxinen: Teil 2: Erfahrungen und Überlegungen. HNO 2007; 55: 485-488

74 Rohrbach S, Gross M. Transnasale fiberoptisch-endoskopische Schluckuntersuchung bei Kindern. Monatsschrift Kinderheilkunde 2014; 162: 329-333

75 Prasse JE, Kikano GE. An overview of pediatric dysphagia. Clin Pediatr (Phila) 2009; 48: 247-251

76 Rommel N, De Meyer AM, Feenstra L, Veereman-Wauters G. The complexity of feeding problems in 700 infants and young children presenting to a tertiary care institution. J Pediatr Gastroenterol Nutr 2003; 37: 75-84

77 Sierra MS, Hastings EV, Goodman KJ. What do we know about benefits of $\mathrm{H}$. pylori treatment in childhood? Gut Microbes 2013; 4: 549-567

78 Al-Hussaini A, Semaan T, El Hag I. Eosinophilic esophagitis in a developing country: is it different from developed countries? Gastroenterol Res Pract 2013; 2013: 526037

79 Bussmann C, Straumann A. Eosinophile Osophagitis: Der diagnostische Beitrag der Pathologie. Pathologe 2013; 34: 110-117

80 Gupta SK. Diagnostic and therapeutic challenges in pediatric eosinophilic esophagitis: past, present and future. Dig Dis 2014; 32: 107109

81 Kühn D, Miller S, Schwemmle C, Jungheim M, Ptok M. Frühkindliches Schlucken. Laryngorhinootologie 2014; 93: 231-236

82 Chatoor I. Fütterstörungen bei Säuglingen und Kleinkindern. Stuttgart: Klett-Cotta, 2012

83 Arvedson JC. Assessment of pediatric dysphagia and feeding disorders: clinical and instrumental approaches. Dev Disabil Res Rev 2008; 14: 118-127

84 Reilly S, Skuse D, Poblete X. Prevalence of feeding problems and oral motor dysfunction in children with cerebral palsy: a community survey. J Pediatr 1996; 129: 877-882

85 Arvedson J, Brodsky L. Pediatric swallowing and feeding. New York: Singular publishing group, 2002

86 Benfer KA, Weir KA, Bell KL, Ware RS, Davies PS, Boyd RN. Oropharyngeal dysphagia and gross motor skills in children with cerebral palsy. Pediatrics 2013; 131: e1553-e1562

87 Calis EA, Veugelers R, Sheppard JJ, Tibboel D, Evenhuis HM, Penning C. Dysphagia in children with severe generalized cerebral palsy and intellectual disability. Dev Med Child Neurol 2008; 50: 625-630

88 Lefton-Greif MA, Arvedson JC. Pediatric feeding and swallowing disorders: state of health, population trends, and application of the international classification of functioning, disability, and health. Semin Speech Lang 2007; 28: 161-165

89 Cowpe E, Hanson B, Smith $C$. What do parents of children with dysphagia think about their MDT? A qualitative study. BMJ Open 2014; 4: e005934

90 Biber D. Frühkindliche Dysphagien und Trinkschwächen. Leitfaden für Diagnostik, Management und Therapie im klinischen Alltag. Wien: Springer-Verlag, 2012

91 Taniguchi $M H$, Moyer RS. Assessment of risk factors for pneumonia in dysphagic children: significance of videofluoroscopic swallowing evaluation. Dev Med Child Neurol 1994; 36: 495-502

92 Gross PA, Neu HC, Aswapokee P, Van Antwerpen C, Aswapokee N. Deaths from nosocomial infections: experience in a university hospital and a community hospital. Am J Med 1980; 68: 219-223

93 Motion S, Northstone K, Emond A, Stucke S, Golding J. Early feeding problems in children with cerebral palsy: weight and neurodevelopmental outcomes. Dev Med Child Neurol 2002; 44: 40-43

94 Newman LA, Keckley C, Petersen MC, Hamner A. Swallowing function and medical diagnoses in infants suspected of Dysphagia. Pediatrics 2001; 108: E106

95 DeMatteo C, Matovich D, Hjartarson A. Comparison of clinical and videofluoroscopic evaluation of children with feeding and swallowing difficulties. Dev Med Child Neurol 2005; 47: 149-157

96 Benfer KA, Weir KA, Boyd RN. Clinimetrics of measures of oropharyngeal dysphagia for preschool children with cerebral palsy and neurodevelopmental disabilities: a systematic review. Dev Med Child Neurol 2012; 54: 784-795

97 Arvedson JC. Swallowing and feeding in infants and young children. GI Motility online 2006, doi:10.1038/gimo1017

98 Rees CJ. Flexible endoscopic evaluation of swallowing with sensory testing. Curr Opin Otolaryngol Head Neck Surg 2006; 14: 425-430

99 Johnson PE, Belafsky PC, Postma GN. Topical nasal anesthesia and laryngopharyngeal sensory testing: a prospective, double-blind crossover study. Ann Otol Rhinol Laryngol 2003; 112: 14-16
100 ASHA. Pediatric Dysphagia. http://www.asha.org/PRPSpecificTopic. aspx?folderid $=8589934965$ \&section $=$ Assessment

101 Gangil A, Patwari AK, Aneja S, Ahuja B, Anand VK. Feeding problems in children with cerebral palsy. Indian Pediatr 2001; 38: 839-846

102 Yilmaz S, Basar P, Gisel EG. Assessment of feeding performance in patients with cerebral palsy. Int J Rehabil Res 2004; 27: 325-329

103 Prosiegel M, Bartolome G, Biniek R, Fheodoroff K, Schlaegel W, Schröter-Morasch H, Saltuari U. Neurogene Dysphagien. In: Diener HC, Putzki N (eds.). Leitlinien für Diagnostik und Therapie in der Neurologie. Stuttgart: Thieme, 2012; 908-919

104 Prosiegel M, Heintze M, Wagner-Sonntag E, Hannig C, Wuttge-Hannig A, Yassouridis A. Schluckstörungen bei neurologischen Patienten. Eine prospektive Studie zu Diagnostik, Störungsmustern, Therapie und Outcome. Nervenarzt 2002; 73: 364-370

105 Weir KA, McMahon SM, Long G, Bunch JA, Pandeya N, Coakley KS, Chang $A B$. Radiation doses to children during modified barium swallow studies. Pediatr Radiol 2007; 37: 283-290

106 Leder SB, Karas DE. Fiberoptic endoscopic evaluation of swallowing in the pediatric population. Laryngoscope 2000; 110: 1132-1136

107 Cohen MA, Setzen M, Perlman PW, Ditkoff M, Mattucci KF, Guss J. The safety of flexible endoscopic evaluation of swallowing with sensory testing in an outpatient otolaryngology setting. Laryngoscope 2003; 113: $21-24$

108 Schröter-Morasch $H$. Klinische Untersuchung des Oropharynx und videoendoskopische Untersuchung der Schluckfunktion. In: Bartolome G, Schröter-Morasch H (eds.). Schluckstörungen: Diagnostik und Rehabilitation. München: Urban und Fischer, 2010

109 Rosenbek JC, Robbins JA, Roecker EB, Coyle JL, Wood JL. A penetration-aspiration scale. Dysphagia 1996; 11: 93-98

110 Willging JP, Thompson DM. Pediatric FEESST: fiberoptic endoscopic evaluation of swallowing with sensory testing. Curr Gastroenterol Rep 2005; 7: 240-243

111 Donzelli J, Brady S, Wesling M, Theisen M. Effects of the removal of the tracheotomy tube on swallowing during the fiberoptic endoscopic exam of the swallow (FEES). Dysphagia 2005; 20: 283-289

112 Carl JJ, Johnson PR. Drugs and Dysphagia. Austin, Texas: Pro-ed, 2006

113 Wright RE, Ellis PK. Patient perception and localization of dysphagia - barium study correlation. Dis Esophagus 1997; 10: 211-214 discussion 215-216

114 Schröter-Morasch H, von Cramon D. Laryngoskopische Befunde bei zentraler traumatischer Dysphonie. HNO 1984; 32: 13-16

115 Morasch H, Joussen K, Ziegler W. Zentrale laryngeale Bewegungsstörungen nach schwerem, gedecktem Schädelhirntrauma und bei zerebrovaskulären Erkrankungen. Laryngol Rhinol Otol (Stuttg) 1987; 66: $214-220$

116 Leder SB, Suiter DM, Murray J, Rademaker AW. Can an oral mechanism examination contribute to the assessment of odds of aspiration? Dysphagia 2013; 28: 370-374

117 Power ML, Hamdy S, Singh S, Tyrrell PJ, Turnbull I, Thompson DG. Deglutitive laryngeal closure in stroke patients. J Neurol Neurosurg Psychiatry 2007; 78: 141-146

118 Oommen ER, Kim Y, McCullough G. Stage transition and laryngeal closure in poststroke patients with dysphagia. Dysphagia 2011; 26: 318-323

119 Ramsey D, Smithard D, Donaldson N, Kalra L. Is the gag reflex useful in the management of swallowing problems in acute stroke? Dysphagia 2005; 20: 105-107

120 Belafsky PC, Mouadeb DA, Rees CJ, Pryor JC, Postma GN, Allen J, Leonard RJ. Validity and reliability of the Eating Assessment Tool (EAT-10). Ann Otol Rhinol Laryngol 2008; 117: 919-924

121 Cheney DM, Siddiqui MT, Litts JK, Kuhn MA, Belafsky PC. The Ability of the 10-Item Eating Assessment Tool (EAT-10) to Predict Aspiration Risk in Persons With Dysphagia. Ann Otol Rhinol Laryngol 2014

122 Dwivedi RC, St Rose S, Roe JW, Khan AS, Pepper C, Nutting CM, Kazi R. Validation of the Sydney Swallow Questionnaire (SSQ) in a cohort of head and neck cancer patients. Oral Oncol 2010; 46: e10-e14

123 Wallace KL, Middleton S, Cook IJ. Development and validation of a self-report symptom inventory to assess the severity of oral-pharyngeal dysphagia. Gastroenterology 2000; 118: 678-687

124 Simons AJ. Munich Dysphagia Test - Parkinson’s Disease (MDT-PD): a new clinical questionnaire for early assessment of dysphagia in Parkinson's disease. 2. Kongress der European Society for Swallowing Disorders. Barcelona, Spanien; 2012

125 Chen AY, Frankowski R, Bishop-Leone J, Hebert T, Leyk S, Lewin J, Goepfert $H$. The development and validation of a dysphagia-specific quality-of-life questionnaire for patients with head and neck cancer: the M. D. Anderson dysphagia inventory. Arch Otolaryngol Head Neck Surg 2001; 127: 870-876 
126 McHorney CA, Bricker DE, Kramer AE, Rosenbek JC, Robbins J, Chignell $K A$, Clarke $C$. The SWAL-QOL outcomes tool for oropharyngeal dysphagia in adults: I. Conceptual foundation and item development. Dysphagia 2000; 15: 115-121

127 McHorney CA, Bricker DE, Robbins J, Kramer AE, Rosenbek JC, Chignell $K A$. The SWAL-QOL outcomes tool for oropharyngeal dysphagia in adults: II. Item reduction and preliminary scaling. Dysphagia 2000; 15: $122-133$

128 McHorney CA, Robbins J, Lomax K, Rosenbek JC, Chignell K, Kramer AE, Bricker DE. The SWAL-QOL and SWAL-CARE outcomes tool for oropharyngeal dysphagia in adults: III. Documentation of reliability and validity. Dysphagia 2002; 17: 97-114

129 Silbergleit AK, Schultz L, Jacobson BH, Beardsley T, Johnson AF. The Dysphagia handicap index: development and validation. Dysphagia 2012; 27: 46-52

130 List MA, D'Antonio LL, Cella DF, Siston A, Mumby P, Haraf D, Vokes E. The Performance Status Scale for Head and Neck Cancer Patients and the Functional Assessment of Cancer Therapy - Head and Neck Scale. A study of utility and validity. Cancer 1996; 77: 2294-2301

131 List MA, Ritter-Sterr CA, Baker TM, Colangelo LA, Matz G, Pauloski BR, Logemann JA. Longitudinal assessment of quality of life in laryngeal cancer patients. Head Neck 1996; 18: 1-10

132 Bartholome G. Aspirationsschnelltests und klinische Schluckuntersuchung. In: Bartholome G, Schröter-Morasch H (eds.). Schluckstörungen - Diagnostik und Rehabilitation. München: Elsevier, 2014

133 Doggett DL, Turkelson CM, Coates V. Recent developments in diagnosis and intervention for aspiration and dysphagia in stroke and other neuromuscular disorders. Curr Atheroscler Rep 2002; 4: 311-318

134 Brady SL, Hildner CD, Hutchins BF. Simultaneous videofluoroscopic swallow study and modified Evans blue dye procedure: An evaluation of blue dye visualization in cases of known aspiration. Dysphagia 1999; 14: 146-149

135 Potts RG, Zaroukian MH, Guerrero PA, Baker CD. Comparison of blue dye visualization and glucose oxidase test strip methods for detecting pulmonary aspiration of enteral feedings in intubated adults. Chest 1993; 103: 117-121

136 Lucarelli MR, Shirk MB, Julian MW, Crouser ED. Toxicity of Food Drug and Cosmetic Blue No. 1 dye in critically ill patients. Chest 2004; 125: 793-795

137 Nathadwarawala KM, Nicklin J, Wiles CM. A timed test of swallowing capacity for neurological patients. J Neurol Neurosurg Psychiatry 1992; 55: 822-825

138 Perry L. Screening swallowing function of patients with acute stroke. Part two: Detailed evaluation of the tool used by nurses. J Clin Nurs 2001; 10: 474-481

139 Perry $L$. Screening swallowing function of patients with acute stroke. Part one: Identification, implementation and initial evaluation of a screening tool for use by nurses. J Clin Nurs 2001; 10: 463-473

140 Trapl M, Enderle P, Nowotny M, Teuschl Y, Matz K, Dachenhausen A, Brainin $M$. Dysphagia bedside screening for acute-stroke patients: the Gugging Swallowing Screen. Stroke 2007; 38: 2948-2952

141 Suiter DM, Leder SB. Clinical utility of the 3-ounce water swallow test. Dysphagia 2008; 23: 244-250

142 DePippo KL, Holas MA, Reding MJ. Validation of the 3-oz water swallow test for aspiration following stroke. Arch Neurol 1992; 49: 1259-1261

143 Kidd D, Lawson J, Nesbitt R, MacMahon J. Aspiration in acute stroke: a clinical study with videofluoroscopy. Q J Med 1993; 86: 825-829

144 DePippo KL, Holas MA, Reding MJ. The Burke dysphagia screening test: validation of its use in patients with stroke. Arch Phys Med Rehabil 1994; 75: 1284-1286

145 Smithard DG, O'Neill PA, Park C, England R, Renwick DS, Wyatt R, North West Dysphagia G. Can bedside assessment reliably exclude aspiration following acute stroke? Age Ageing 1998; 27: 99-106

146 Massey R, Jedlicka D. The Massey Bedside Swallowing Screen. J Neurosci Nurs 2002; 34: 252-253, 257-260

147 Nishiwaki K, Tsuji T, Liu M, Hase K, Tanaka N, Fujiwara T. Identification of a simple screening tool for dysphagia in patients with stroke using factor analysis of multiple dysphagia variables. J Rehabil Med 2005; 37: 247-251

148 Clave P, Arreola V, Romea M, Medina L, Palomera E, Serra-Prat M. Accuracy of the volume-viscosity swallow test for clinical screening of oropharyngeal dysphagia and aspiration. Clin Nutr 2008; 27: 806-815

149 Martino R, Silver F, Teasell R, Bayley M, Nicholson G, Streiner DL, Diamant NE. The Toronto Bedside Swallowing Screening Test (TORBSST): development and validation of a dysphagia screening tool for patients with stroke. Stroke 2009; 40: 555-561
150 Antonios N, Carnaby-Mann G, Crary M, Miller L, Hubbard H, Hood K, Silliman $S$. Analysis of a physician tool for evaluating dysphagia on an inpatient stroke unit: the modified Mann Assessment of Swallowing Ability. J Stroke Cerebrovasc Dis 2010; 19: 49-57

151 Edmiaston J, Connor LT, Loehr L, Nassief A. Validation of a dysphagia screening tool in acute stroke patients. Am J Crit Care 2010; 19: 357-364

152 Wakasugi Y, Tohara H, Nakane A, Murata S, Mikushi S, Susa C, Uemat$s u \mathrm{H}$. Usefulness of a handheld nebulizer in cough test to screen for silent aspiration. Odontology 2014; 102: 76-80

153 Trapl M, Enderle P, Nowotny M, Teuschl Y, Matz K, Dachenhausen A Brainin $M$. Dysphagia bedside screening for acute-stroke patients: the Gugging Swallowing Screen 2008 http://www.donau-uni.ac.at/ imperia/md/images/department/kmp/publikationen/guss_d.pdf

154 Hey C, Lange B, Eberle S, Stöver T. Entwicklung und Validierung des Frankfurter Dysphagiescreenings (FraDySc) für Patienten mit KopfHals-Tumor nach Tumorresektion. Itemreduktion und erste Validierung 29. Wissenschaftliche Jahrestagung der Deutschen Gesellschaft für Phoniatrie und Pädaudiologie. Bonn: gms, 21.9.-23.92012

155 Hey C, Lange BP, Eberle S, Zaretsky Y, Sader R, Stover T, Wagenblast J. Water swallow screening test for patients after surgery for head and neck cancer: early identification of dysphagia, aspiration and limitations of oral intake. Anticancer Res 2013; 33: 4017-4021

156 Carnaby GD, Crary MA. Development and validation of a cancer-specific swallowing assessment tool: MASA-C. Support Care Cancer 2014; 22: 595-602

157 Sasaki CT, Leder SB. Comments on selected recent Dysphagia literature. Dysphagia 2014; 29: 731-736

158 Logemann JA. Evaluation and Treatment of Swallowing Disorders. Austin, Texas: pro-ed, 1998

159 Murray J. The clinical swallowing examination. In: Murray J (ed.). Manual of Dysphagia Assessment in Adults. San Diego,CA: Singular, 1999; 37-112

160 Murry T, Carrau RL. Clinical managment of swallowing disorders. Plural Publishing. 2012

161 Mathers-Schmidt BA, Kurlinski M. Dysphagia evaluation practices: inconsistencies in clinical assessment and instrumental examination decision-making. Dysphagia 2003; 18: 114-125

162 Birkmann $U$. Kö.Be.S: Kölner Befundsystem für Schluckstörungen. ProLog 2007

163 Ickenstein GW, Hofmayer A, Lindner-Pfleghar B, Pluschinski P, Riecker A, Schelling A, Prosiegel M. Standardisierung des Untersuchungsablaufs bei Neurogener Oropharyngealer Dysphagie (NOD). Neurologie und Rehabilitation 2009; 15: 290-300

164 Stix S.Dysphagie-Screening zur Erfassung neurogener Dysphagien [Abschlussarbeit ÖÄK Diplomlehrgang Geriatrie]. 2009; http://www. arztakademie.at/fileadmin/template/main/Geriatrie/Publikationen 09-10/Stix.pdf

165 Langmore SE. Evaluation of oropharyngeal dysphagia: which diagnostic tool is superior? Curr Opin Otolaryngol Head Neck Surg 2003; 11: 485-489

166 Bastian RW. Videoendoscopic evaluation of patients with dysphagia: an adjunct to the modified barium swallow. Otolaryngol Head Neck Surg 1991; 104: 339-350

167 Bastian RW. The videoendoscopic swallowing study: an alternative and partner to the videofluoroscopic swallowing study. Dysphagia 1993; 8: 359-367

168 Schröter-Morasch $H$. Klinische Untersuchung der am Schluckvorgang beteiligten Organe. In: Bartolome G (ed.). Diagnostik und Therapie neurologisch bedingter Schluckstörungen. München: G. Fischer, 1993; 73-108

169 Butler SG, Stuart A, Markley L, Rees C. Penetration and aspiration in healthy older adults as assessed during endoscopic evaluation of swallowing. Ann Otol Rhinol Laryngol 2009; 118: 190-198

170 Kelly AM, Leslie P, Beale T, Payten C, Drinnan MJ. Fibreoptic endoscopic evaluation of swallowing and videofluoroscopy: does examination type influence perception of pharyngeal residue severity? Clin Otolaryngol 2006; 31: 425-432

171 Colodny $N$. Interjudge and intrajudge reliabilities in fiberoptic endoscopic evaluation of swallowing (fees) using the penetration-aspiration scale: a replication study. Dysphagia 2002; 17: 308-315

172 Aviv JE. Prospective, randomized outcome study of endoscopy versus modified barium swallow in patients with dysphagia. Laryngoscope 2000; 110: 563-574

173 Leder SB, Sasaki CT, Burrell MI. Fiberoptic endoscopic evaluation of dysphagia to identify silent aspiration. Dysphagia 1998; 13: 19-21

174 Kaye GM, Zorowitz RD, Baredes S. Role of flexible laryngoscopy in evaluating aspiration. Ann Otol Rhinol Laryngol 1997; 106: 705-709 
175 Wu CH, Hsiao TY, Chen JC, Chang YC, Lee SY. Evaluation of swallowing safety with fiberoptic endoscope: comparison with videofluoroscopic technique. Laryngoscope 1997; 107: 396-401

176 Kidder TM, Langmore SE, Martin BJ. Indications and techniques of endoscopy in evaluation of cervical dysphagia: comparison with radiographic techniques. Dysphagia 1994; 9: 256-261

177 Langmore SE, Schatz K, Olson N. Endoscopic and videofluoroscopic evaluations of swallowing and aspiration. Ann Otol Rhinol Laryngol 1991; 100: 678-681

178 Schröter-Morasch H, Bartolome G, Troppmann N, Ziegler W. Values and limitations of pharyngolaryngoscopy (transnasal, transoral) in patients with dysphagia. Folia Phoniatr Logop 1999; 51: 172-182

179 Doggett DL, Tappe KA, Mitchell MD, Chapell R, Coates V, Turkelson CM. Prevention of pneumonia in elderly stroke patients by systematic diagnosis and treatment of dysphagia: an evidence-based comprehensive analysis of the literature. Dysphagia 2001; 16: 279-295

180 Aviv JE, Kim T, Sacco RL, Kaplan S, Goodhart K, Diamond B, Close LG. FEESST: a new bedside endoscopic test of the motor and sensory components of swallowing. Ann Otol Rhinol Laryngol 1998; 107: 378-387

181 Wada $H$, Nakajoh K, Satoh-Nakagawa T, Suzuki T, Ohrui T, Arai $H$, Sasaki $H$. Risk factors of aspiration pneumonia in Alzheimer's disease patients. Gerontology 2001; 47: 271-276

182 Gallenberger S, Schröter-Morasch $H$. Diagnostische und therapeutische Aspekte der Bronchoskopie bei aspirations-gefährdeten Patienten. Sprache Stimme Gehör 1999; 23: 32-34

183 Anagnostopoulos-Schleep J, Franz M, Lehmann J. Videoendoskopische Pharyngolaryngoskopie: Untersuchungstechnik und Befundinterpretation. Neurol Rehabil 1999; 5: 133-141

184 Herrmann IF, Arce-Recio S. Functional pharyngoesophagoscopy: a new technique for diagnostics and analyzing deglutition. Operative Techniques in Otolaryngology - Head and Neck Surgery 1997; 8: 163-167

185 Herrmann IF, Siegersma E, Arce-Recio S, Pedicino A. Endoskopie der pharyngealen Phase des Schluckaktes. Oto-Rhino-Laryngologia Nova 1996; 6: 7-14

186 Ajemian MS, Nirmul GB, Anderson MT, Zirlen DM, Kwasnik EM. Routine fiberoptic endoscopic evaluation of swallowing following prolonged intubation: implications for management. Arch Surg 2001; 136: 434-437

187 Leder SB, Cohn SM, Moller BA. Fiberoptic endoscopic documentation of the high incidence of aspiration following extubation in critically ill trauma patients. Dysphagia 1998; 13: 208-212

188 Diesener $P$. Schluckstörungen bei Kindern: Bildgebende Diagnostik mittels transnasaler Videoendoskopie. Prakt Pädiatrie 1999; 5: 36-42

189 Migliore LE, Scoopo FJ, Robey KL. Fiberoptic Examination of Swallowing in Children and Young Adults With Severe Developmental Disability. American Journal of Speech-Language Pathology 1999; 8: 303-308

190 Keller J. Endoskopische Charakteristik oropharyngealer Dysphagien bei unterschiedlichen Demenzformen. Dysphagieforum 2012; 1: 29-33

191 Deutschmann MW, McDonough A, Dort JC, Dort E, Nakoneshny S, Matthews TW. Fiber-optic endoscopic evaluation of swallowing (FEES): predictor of swallowing-related complications in the head and neck cancer population. Head Neck 2013; 35: 974-979

192 Keller J, Durwen HF. Die fiberendoskopische Evaluation des Schluckens (FEES) in der Geriatrie - mit besonderer Berücksichtigung des akuten Schlaganfalls. NeuroGeriatrie 2010; 7: 59-64

193 Barczi SR, Sullivan PA, Robbins J. How should dysphagia care of older adults differ? Establishing optimal practice patterns. Semin Speech Lang 2000; 21: 347-361

194 Rehman HU, Knox J. There is a need for a regular review of swallowing ability in patients after PEG insertion to identify patients with delayed recovery of swallowing. Dysphagia 2000; 15: 48

195 Bastian RW, Nagorsky MJ. Laryngeal image biofeedback. Laryngoscope 1987; 97: 1346-1349

196 Denk DM, Kaider A. Videoendoscopic biofeedback: a simple method to improve the efficacy of swallowing rehabilitation of patients after head and neck surgery. ORL J Otorhinolaryngol Relat Spec 1997; 59: 100-105

197 Aviv JE, Kaplan ST, Thomson JE, Spitzer J, Diamond B, Close LG. The safety of flexible endoscopic evaluation of swallowing with sensory testing (FEESST): an analysis of 500 consecutive evaluations. Dysphagia 2000; 15: 39-44

198 Aviv JE, Murry T, Zschommler A, Cohen M, Gartner C. Flexible endoscopic evaluation of swallowing with sensory testing: patient characteristics and analysis of safety in 1,340 consecutive examinations. Ann Otol Rhinol Laryngol 2005; 114: 173-176
199 Mellies JK. Die Fiberoptische Endoskopische Evaluation des Schluckens (FEES), eine wertvolle Methode zur Untersuchung von Dysphagiepatienten. Neurophysiol Lab 2008

200 Schindler A, Ginocchio D, Peri A, Felisati G, Ottaviani F. FEESST in the rehabilitation of dysphagia after partial laryngectomy. Ann Otol Rhinol Laryngol 2010; 119: 71-76

201 Aviv JE, Martin JH, Sacco RL, Zagar D, Diamond B, Keen MS, Blitzer A. Supraglottic and pharyngeal sensory abnormalities in stroke patients with dysphagia. Ann Otol Rhinol Laryngol 1996; 105: 92-97

202 Aviv JE, Sacco RL, Thomson J, Tandon R, Diamond B, Martin JH, Close $L G$. Silent laryngopharyngeal sensory deficits after stroke. Ann Otol Rhinol Laryngol 1997; 106: 87-93

203 Aviv JE, Spitzer J, Cohen M, Ma G, Belafsky P, Close LG. Laryngeal adductor reflex and pharyngeal squeeze as predictors of laryngeal penetration and aspiration. Laryngoscope 2002; 112: 338-341

204 Seidl RO, N-M-B R, Ernst A. Evaluation eines Untersuchungsbogens zur endoskopischen Schluckuntersuchung. Sprache Stimme Gehör 2002; 26: 28-36

205 Dziewas $R$, Warnecke T, Olenberg S, Teismann I, Zimmermann J, Kramer $C$, Schabitz WR. Towards a basic endoscopic assessment of swallowing in acute stroke - development and evaluation of a simple dysphagia score. Cerebrovasc Dis 2008; 26: 41-47

206 Schröter-Morasch H, Graf S. Dysphagiediagnostik durch den HNOArzt. HNO 2014; 62: 324-334

207 Sasaki CT, Hundal JS, Kim YH. Protective glottic closure: biomechanical effects of selective laryngeal denervation. Ann Otol Rhinol Laryngol 2005; 114: 271-275

208 Malandraki GA, Hind JA, Gangnon R, Logemann JA, Robbins J. The utility of pitch elevation in the evaluation of oropharyngeal Dysphagia: preliminary findings. Am J Speech Lang Pathol 2011; 20: 262-268

209 Fuller SC, Leonard R, Aminpour S, Belafsky PC. Validation of the pharyngeal squeeze maneuver. Otolaryngol Head Neck Surg 2009; 140: 391-394

210 Rodriguez $K H$, Roth CR, Rees CJ, Belafsky PC. Reliability of the pharyngeal squeeze maneuver. Ann Otol Rhinol Laryngol 2007; 116: 399-401

211 Martin BJ, Logemann JA, Shaker R, Dodds WJ. Normal laryngeal valving patterns during three breath-hold maneuvers: a pilot investigation. Dysphagia 1993; 8: 11-20

212 Ohmae Y, Ogura M, Kitahara S, Karaho T, Inouye T. Effects of head rotation on pharyngeal function during normal swallow. Ann Otol Rhinol Laryngol 1998; 107: 344-348

213 Setzen M, Cohen MA, Perlman PW, Belafsky PC, Guss J, Mattucci KF Ditkoff $M$. The association between laryngopharyngeal sensory deficits, pharyngeal motor function, and the prevalence of aspiration with thin liquids. Otolaryngol Head Neck Surg 2003; 128: 99-102

214 Hacki T, Kramer H, Kleinjung C, Perez-Alvarez C, Schmid J. Endoskopische Mehrfarben-Schluckuntersuchung. Laryngorhinootologie 2001; 80: $335-340$

215 Robbins J, Coyle J, Rosenbek J, Roecker E, Wood J. Differentiation of normal and abnormal airway protection during swallowing using the penetration-aspiration scale. Dysphagia 1999; 14: 228-232

216 Hey C, Pluschinski P, Zaretsky Y, Almahameed A, Hirth D, Vaerst B, Stöver T. Penetrations-Aspirations-Skala nach Rosenbek. Validierung der deutschen Version für die endoskopische Dysphagiediagnostik. HNO 2014; 62: 276-281

217 Langmore SE. Scoring a FEES examination. In: Langmore SE (ed.). Endoscopic evaluation and treatment of swallowing disorders. New York: Thieme, 2001; 101-143

218 Hey C, Sader RA, Belogradski D, Pluschinski P, Stanschus S, Euler HA, Neumann $K$. Computer-assisted documentation of the fiberoptic endoscopic evaluation of swallowing. Med Sci Monit 2009; 15: MT41-MT46

219 Starrost U, Bartolome G, Schröter-Morasch H, Ziegler W, Fussenegger C, Marano C, Schilling BK. Der Bogenhausener Dysphagiescore - BODS: Inhaltsvalidität und Reliabilität. DysphagieForum 2012; 2: 2-11

220 Crary MA, Mann GD, Groher ME. Initial psychometric assessment of a functional oral intake scale for dysphagia in stroke patients. Arch Phys Med Rehabil 2005; 86: 1516-1520

221 Schröter-Morasch $H$. Schweregradeinteilung der Aspiration bei Patienten mit Schluckstörung. In: Gross M.ed. Aktuelle phoniatrischpädaudiologische Aspekte. Berlin: R.Gross Verlag, 1996; 145-146

222 Hannig C, Wuttge-Hannig A, Hess $U$. Analyse und radiologisches Staging des Typs und Schweregrades einer Aspiration. Radiologe 1995; 35: 741-746

223 Farneti $D$. Pooling score: an endoscopic model for evaluating severity of dysphagia. Acta Otorhinolaryngol Ital 2008; 28: 135-140

224 Pluschinski P, Zaretsky Y, Almahameed A, Koseki JC, Leinung M, Girth $L$, Hey $C$. Sekretbeurteilungsskala nach Murray et al. fur $\operatorname{FEES}((\mathrm{R}))$ : Reliabilitäts- und Validitätsvergleich der deutschen Lang- und Kurzversion. Nervenarzt 2014; 85: 1582-1587 
225 Fujiu M, Logemann JA. Effect of a Tongue-Holding Maneuver on Posterior Pharyngeal Wall Movement During Deglutition. American Journal of Speech-Language Pathology 1996; 5: 23-30

226 Shaker R, Easterling C, Kern M, Nitschke T, Massey B, Daniels S, Dikeman $K$. Rehabilitation of swallowing by exercise in tube-fed patients with pharyngeal dysphagia secondary to abnormal UES opening. Gastroenterology 2002; 122: 1314-1321

227 Jacob P, Kahrilas PJ, Logemann JA, Shah V, Ha T. Upper esophageal sphincter opening and modulation during swallowing. Gastroenterology 1989; 97: 1469-1478

228 Hirst LJ, Sama A, Carding PN, Wilson JA. Is a 'safe swallow' really safe? Int J Lang Commun Disord 1998; 33: (Suppl): 279-280

229 Welch MV, Logemann JA, Rademaker AW, Kahrilas PJ. Changes in pharyngeal dimensions effected by chin tuck. Arch Phys Med Rehabil 1993; 74: 178-181

230 Logemann JA, Kahrilas PJ, Kobara M, Vakil NB. The benefit of head rotation on pharyngoesophageal dysphagia. Arch Phys Med Rehabil 1989; 70: 767-771

231 Neumann S, Bartolome G, Buchholz D, Prosiegel M. Swallowing therapy of neurologic patients: correlation of outcome with pretreatment variables and therapeutic methods. Dysphagia 1995; 10: 1-5

232 Carnaby G, Hankey GJ, Pizzi J. Behavioural intervention for dysphagia in acute stroke: a randomised controlled trial. Lancet Neurol 2006; 5: 31-37

233 Nusser-Müller-Busch R. Die Therapie des Facio-oralen Trakts. Berlin Heidelberg New York: Springer Verlag, 2011

234 Frank U, Mader M, Sticher H. Dysphagic patients with tracheotomies: a multidisciplinary approach to treatment and decannulation management. Dysphagia 2007; 22: 20-29

235 Gerber ME, Gaugler MD, Myer CM $3^{\text {rd }}$, Cotton RT. Chronic aspiration in children. When are bilateral submandibular gland excision and parotid duct ligation indicated? Arch Otolaryngol Head Neck Surg 1996; 122: 1368-1371

236 Noonan K, Prunty S, Ha JF, Vijayasekaran S. Surgical management of chronic salivary aspiration. Int J Pediatr Otorhinolaryngol 2014; 78: 2079-2082

237 Leitlinie_DGHNOKHC. Hypersalivation. http://www.awmf.org/uploads/ tx_szleitlinien/017-0751_S2k_Hypersalivation_2013-02.pdf AWMF online, $01 / 2013$

238 Gadebusch-Bondio M, Herrmann IF, Montani MC. Innenansichten des Krankseins. Berlin: Logos-Verlag, 2013

239 Herrmann IF, Meisel M, Strahl HM. Die neue Videopanendoskopie flexibel, hygienisch und preiswert. Forum HNO 2012; 208-212

240 Fisher DM, Sommerlad BC. Cleft lip, cleft palate, and velopharyngeal insufficiency. Plast Reconstr Surg 2011; 128: 342e-360e

241 Kokemüller H, Essig H, Rücker M, Gellrich $N$-C. Operative Behandlung von Gaumenspalten und velopharyngealer Insuffizienz. Sprache • Stimme • Gehör 2013; 36: 70-72

242 Bishop A, Hong P, Bezuhly M. Autologous fat grafting for the treatment of velopharyngeal insufficiency: state of the art. J Plast Reconstr Aesthet Surg 2014; 67: 1-8

243 Herrmann IF. Entzündungen und sonstige Erkrankungen im Rachen. In: Naumann HH, Helms J, Herberhold C, Kastenbauer E (eds.). Oto-RhinoLaryngologie in Klinik und Praxis, Band 2. Stuttgart: Georg Thieme Verlag, 1992; 608-626

244 Balbuena L Jr, Hayes D, Ramirez SG, Johnson R. Eagle's syndrome (elongated styloid process). South Med J 1997; 90: 331-334

245 Torres AC, Guerrero JS, Silva HC. A modified transoral approach for carotid artery type Eagle syndrome: technique and outcomes. Ann Otol Rhinol Laryngol 2014; 123: 831-834

246 Lecerf $P$, Malard 0 . How to diagnose and treat symptomatic anterior cervical osteophytes? Eur Ann Otorhinolaryngol Head Neck Dis 2010; 127: 111-116

247 Herrmann IF, Arce-Recio S. Special techniques for resolving aspiration problems. Operative Techniques in Otolaryngology - Head and Neck Surgery 1998; 9: 180-191

248 Calcaterra TC. Laryngeal suspension after supraglottic laryngectomy. Arch Otolaryngol 1971; 94: 306-309

249 Hillel $A D$, Goode RL. Lateral laryngeal suspension: a new procedure to minimize swallowing disorders following tongue base resection. Laryngoscope 1983; 93: 26-31

250 Herrmann IF. Surgical solutions for aspiration problems. J Jpn Bronchoesophagol Soc 1992; 43: 72-79

251 Mahieu HF, de Bree R, Westerveld G-J, Leemans CR. Laryngeal suspension and upper esophageal sphincter myotomy as a surgical option for treatment of severe aspiration. Operative Techniques in Otolaryngology - Head and Neck Surgery 1999; 10: 305-310

252 Kos MP. Surgery for dysphagia and aspiration. Academisch Proefschrift. Amsterdam: Vrije Universiteit Amsterdam, 2011; 45-87
253 Laurian N, Shvili Y, Zohar Y. Epiglotto-aryepiglottopexy: a surgical procedure for severe aspiration. Laryngoscope 1986; 96: 78-81

254 Voigt-Zimmermann S, Arens C. Behandlung von Glottisschlussinsuffizienzen. HNO 2013; 61: 117-134

255 Giraldez-Rodriguez LA, Johns $M 3^{\text {rd }}$. Glottal insufficiency with aspiration risk in dysphagia. Otolaryngol Clin North Am 2013; 46: 1113-1121

256 Isshiki N. Progress in laryngeal framework surgery. Acta Otolaryngol 2000; 120: 120-127

257 Nawka T, Hosemann W. Gestörte Stimme: Chirurgische Verfahren. Laryngorhinootologie 2005; 84 (Suppl 1): S201-S212

258 Herrmann IF. Glottoplasty with functional pharynx surgery and tracheostomaplasty. In: Herrmann IF (ed.). Speech restoration via voice prosthesis. Springer Verlag, 1986; 116-124

259 Herrmann IF. Die sekundäre chirurgische Stimmrehabilitation. HNO 1987; 35: 351-354

260 Eisele DW, Yarington CT Jr, Lindeman RC, Larrabee WF Jr. The tracheoesophageal diversion and laryngotracheal separation procedures for treatment of intractable aspiration. Am J Surg 1989; 157: 230-236

261 Zocratto OB, Savassi-Rocha PR, Paixao RM, Salles JM. Laryngotracheal separation surgery: outcome in 60 patients. Otolaryngol Head Neck Surg 2006; 135: 571-575

262 Hara H, Hori T, Sugahara K, Ikeda T, Kajimoto M, Yamashita H. Effectiveness of laryngotracheal separation in neurologically impaired pediatric patients. Acta Otolaryngol 2014; 134: 626-630

263 Ford CN, Samlan R, Robbins J. Voice restoration by traheo-tracheolaryngeal shunt after laryngotracheal diversion for chronic aspiration. Operative Techniques in Otolaryngology - Head and Neck Surgery 1999; 10: 303-304

264 Burnip E, Owen SJ, Barker S, Patterson JM. Swallowing outcomes following surgical and non-surgical treatment for advanced laryngeal cancer. J Laryngol Otol 2013; 127: 1116-1121

265 Sweeny L, Golden JB, White HN, Magnuson JS, Carroll WR, Rosenthal $E L$. Incidence and outcomes of stricture formation postlaryngectomy. Otolaryngol Head Neck Surg 2012; 146: 395-402

266 Hutcheson KA, Alvarez CP, Barringer DA, Kupferman ME, Lapine PR, Lewin JS. Outcomes of elective total laryngectomy for laryngopharyngeal dysfunction in disease-free head and neck cancer survivors. Otolaryngol Head Neck Surg 2012; 146: 585-590

267 Cheung NH, Napolitano LM. Tracheostomy: epidemiology, indications, timing, technique, and outcomes. Respir Care 2014; 59: 895-915 discussion 916-899

268 Durbin CG Jr, Perkins MP, Moores LK. Should tracheostomy be performed as early as $72 \mathrm{~h}$ in patients requiring prolonged mechanical ventilation? Respir Care 2010; 55: 76-87

269 Durbin CG Jr. Tracheostomy: why, when, and how? Respir Care 2010; 55: 1056-1068

270 De Leyn P, Bedert L, Delcroix M, Depuydt P, Lauwers G, Sokolov Y, Belgian Association of Cardiothoracic S. Tracheotomy: clinical review and guidelines. Eur J Cardiothorac Surg 2007; 32: 412-421

271 Weissbrod PA, Merati AL. Is percutaneous dilational tracheotomy equivalent to traditional open surgical tracheotomy with regard to perioperative and postoperative complications? Laryngoscope 2012; 122: $1423-1424$

272 Bast F, Buchal A, Schrom T. Perkutane Dilatationstracheotomie oder Tracheostomie?: 2 Kasuistiken. HNO 2014

273 Bause H, Dost P, Kehrl W, Walz MK, Schultz-Coulon HJ. Punktionstracheotomie versus konventionelle Tracheostomie. Ein interdisziplinäres Gespräch. HNO 1999; 47: 58-70

274 McWhorter AJ. Tracheotomy: timing and techniques. Curr Opin Otolaryngol Head Neck Surg 2003; 11: 473-479

275 Mitchell RB, Hussey HM, Setzen G, Jacobs IN, Nussenbaum B, Dawson $C$, Merati A. Clinical consensus statement: tracheostomy care. Otolaryngol Head Neck Surg 2013; 148: 6-20

276 Kurien M, McAlindon ME, Westaby D, Sanders DS. Percutaneous endoscopic gastrostomy (PEG) feeding. BMJ 2010; 340: c2414

277 Mays AC, Moustafa F, Worley M, Waltonen JD, D'Agostino R Jr. A model for predicting gastrostomy tube placement in patients undergoing surgery for upper aerodigestive tract lesions. JAMA Otolaryngol Head Neck Surg 2014; 140: 1198-1206

278 Bundesärztekammer, Kassenärztliche Vereinigung. Persönliche Leistungserbringung - Möglichkeiten und Grenzen der Delegation ärztlicher Leistungen, 2008: 6, http://www.bundesaerztekammer.de/ page.asp?his $=0.7 .47 .3225$

279 Herrmann IF. Die Pharyxrekonstruktion mit dem Platysma-Faszienlappen - vorläufige Mitteilung. Laryngorhinootologie 1984; 63: 274-276

280 Herrmann IF, Domagk D, Strahl M, Arens C. The transnasal Videopanendoscopy ( $\mathrm{ViP}$ ): diagnostics of the upper aerodigestive tract using an anatomically correct model from the model to the patient. Eur Arch Otorhinolaryngol 2015 Feb 27 